\title{
Casimir-Polder forces: a nonperturbative approach
}

\author{
Stefan Yoshi Buhmann * Ludwig Knöll, and Dirk-Gunnar Welsch \\ Theoretisch-Physikalisches Institut, Friedrich-Schiller-Universität Jena, Max-Wien-Platz 1, 07743 Jena, Germany \\ Ho Trung Dung \\ Institute of Physics, National Center for Sciences and Technology, \\ 1 Mac Dinh Chi Street, District 1, Ho Chi Minh city, Vietnam
}

(Dated: November 13, 2018)

\begin{abstract}
Within the frame of macroscopic QED in linear, causal media, we study the radiation force of Casimir-Polder type acting on an atom which is positioned near dispersing and absorbing magnetodielectric bodies and initially prepared in an arbitrary electronic state. It is shown that minimal and multipolar coupling lead to essentially the same lowest-order perturbative result for the force acting on an atom in an energy eigenstate. To go beyond perturbation theory, the calculations are based on the exact center-of-mass equation of motion. For a nondriven atom in the weak-coupling regime, the force as a function of time is a superposition of force components that are related to the electronic density-matrix elements at a chosen time. Even the force component associated with the ground state is not derivable from a potential in the ususal way, because of the position dependence of the atomic polarizability. Further, when the atom is initially prepared in a coherent superposition of energy eigenstates, then temporally oscillating force components are observed, which are due to the interaction of the atom with both electric and magnetic fields.

PACS numbers: $12.20 .-\mathrm{m}, 42.50 . \mathrm{Vk}, 42.50 . \mathrm{Nn}, 32.70 . \mathrm{Jz}$
\end{abstract}

\section{INTRODUCTION}

It is well known that in the presence of macroscopic bodies an atom in the ground state (or in an excited energy eigenstate) is subject to a nonvanishing force - the Casimir-Polder (CP) force - that results from the vacuum fluctuations of the electromagnetic field. CP forces play an important role in a variety of processes in physical chemistry, atom optics, and cavity QED. Moreover, they hold the key to a number of potential applications in nanotechnology such as the construction of atomic-force microscopes [1] or reflective atom-optical elements [2]. Over the years, substantial efforts have been made to improve the understanding of CP forces (for reviews, see Ref. [3] ). Measuring $\mathrm{CP}$ forces acting on individual particles is a challenging task. Since the early observation of the deflection of thermal atomic beams by conducting surfaces [4], measurement techniques and precision have been improving continuously. More recent experiments have been performed with atomic beams traversing between parallel plates [5]. Other methods include transmission grating diffraction of molecular beams [6], atomic quantum reflection [7, 8], evanescent-wave atomic mirror techniques [9], and indirect measurements via spectroscopic means [10]. Proposals have been made on improvements of monitoring the $\mathrm{CP}$ interaction by using atomic interferometry [11].

The theoretical approaches to the problem of determining the $\mathrm{CP}$ force can be roughly divided into two categories. In the first, first-principle approach explicit field

*Electronic address: s.buhmann@tpi.uni-jena.de quantization is performed and perturbation theory is applied to calculate the body-induced atomic energy shift, which is regarded as the potential of the force in lowestorder perturbation theory [12, 13, 14, 15, 16, 17, 18, 19]. The calculations have typically been based on macroscopic QED, by beginning with a normal-mode decomposition and including the bodies via the well-known conditions of continuity at the surfaces of discontinuity. Since in such a (noncausal) approach the frequency dependence of the bodies' response to the field cannot be properly taken into account, material dispersion and absorption are commonly ignored. As has been shown recently [20], the problem does not occur within the frame of a generalized quantization scheme that properly takes into account a Kramers-Kronig consistent response of the bodies to the field. Clearly, the problem can also be circumvented in microscopic QED, where the bodies are treated on a microscopic level by adopting, e.g., harmonic-oscillator models [14]. In the second, semiphenomenological approach, the problem is circumvented by basing the calculations on linear response theory (LRT), without explicitly quantizing the electromagnetic field $21,22,23,24,25,26,27,28]$. In the ansatz for the force, either the field quantities or both the field and the atomic entities are expressed in terms of correlation functions, which in turn are related, via the fluctuation-dissipation theorem, to response functions.

At first glance one would expect the result obtained from exploiting LRT to be more generally valid than the QED result obtained in lowest-order perturbation theory. In fact, this is not the case. In both approaches, it is not the exact atomic polarizability that enters the expression for the (ground-state) CP force but the approximate expression which is obtained in lowest-order 
perturbation theory and which effectively corresponds to the atomic polarizability in free space. Since the structure of the electromagnetic field is changed in the presence of macroscopic bodies, the atomic polarizability is expected to change as well. It is well known that the atomic level shifts and broadenings sensitively depend on the material surroundings. In particular, when an atom is situated very close to a body, the effect can be quite significant (see, e.g., Refs. [29, 30]), thereby changing the atomic polarizability. As a result, a position-dependent polarizability is expected to occur, which prevents the CP force from being derivable from a potential in the usual way.

A way to derive a more rigorous expression for the $\mathrm{CP}$ force is to base the calculations on the exact quantummechanical center-of-mass equation of motion of the atom as we shall do in this paper. The calculations are performed for both minimal and multipolar coupling, and contact is made with earlier studies of the center-of-mass motion of an atom in free space, with special emphasis on the so-called Röntgen interaction term that appears in the multipolar Hamiltonian [31, 32, 33, 34, 35]. After taking the expectation value with respect to the internal (electronic) quantum state of the atom and the quantum state of the medium-assisted electromagnetic field, the resulting force formula can be used to calculate the time-dependent force acting on a nondriven or driven atom that is initially prepared in an arbitrary (internal) quantum state. In this paper, the force formula is further evaluated for the case of a nondriven, initially arbitrarily prepared atom, by assuming weak atom-field coupling treated in Markovian approximation. It is worth noting that the theory, being based on the quantized version of the macroscopic Maxwell field, with the bodies being described in terms of spatially varying, KramersKronig consistent complex permittivities and permeabilities [36, 37], also applies to left-handed materials [38] where standard quantization runs into difficulties.

The paper is organized as follows. After a brief sketch of the quantization scheme (Sec. III), in Sec. III attention is focused on the perturbative treatment of the $\mathrm{CP}$ force acting on an atom in an energy eigenstate, and previous results [20] obtained for dielectric surroundings of the atom are extended to magnetodielectric surroundings, including left-handed materials. In Sec.IV the exact center-of-mass Heisenberg equation of motion of an atom and the Lorentz force therein are studied, and Sec. $\nabla$ is devoted to the calculation of the average force, with special emphasis on a nondriven atom in the weak-coupling regime. Finally, a summary and some concluding remarks are given in Sec. VI

\section{SKETCH OF THE QUANTIZATION SCHEME}

\section{A. Minimal coupling}

In Coulomb gauge, the minimal-coupling Hamiltonian of an atomic system (e.g., an atom or a molecule) consisting of nonrelativistic charged particles interacting with the electromagnetic field in the presence of macroscopic dispersing and absorbing bodies reads 37]

$$
\begin{aligned}
\hat{H}= & \sum_{\lambda=e, m} \int \mathrm{d}^{3} r \int_{0}^{\infty} \mathrm{d} \omega \hbar \omega \hat{\mathbf{f}}_{\lambda}^{\dagger}(\mathbf{r}, \omega) \hat{\mathbf{f}}_{\lambda}(\mathbf{r}, \omega) \\
& +\sum_{\alpha} \frac{1}{2 m_{\alpha}}\left[\hat{\mathbf{p}}_{\alpha}-q_{\alpha} \hat{\mathbf{A}}\left(\hat{\mathbf{r}}_{\alpha}\right)\right]^{2} \\
& +\frac{1}{2} \int \mathrm{d}^{3} r \hat{\rho}_{A}(\mathbf{r}) \hat{\varphi}_{\mathrm{A}}(\mathbf{r})+\int \mathrm{d}^{3} r \hat{\rho}_{A}(\mathbf{r}) \hat{\varphi}(\mathbf{r}),
\end{aligned}
$$

where

$$
\hat{\rho}_{\mathrm{A}}(\mathbf{r})=\sum_{\alpha} q_{\alpha} \delta\left(\mathbf{r}-\hat{\mathbf{r}}_{\alpha}\right)
$$

and

$$
\hat{\varphi}_{\mathrm{A}}(\mathbf{r})=\int \mathrm{d}^{3} r^{\prime} \frac{\hat{\rho}_{\mathrm{A}}\left(\mathbf{r}^{\prime}\right)}{4 \pi \varepsilon_{0}\left|\mathbf{r}-\mathbf{r}^{\prime}\right|}
$$

are the charge density and scalar potential of the particles, respectively. The particle labeled $\alpha$ has charge $q_{\alpha}$, mass $m_{\alpha}$, position $\hat{\mathbf{r}}_{\alpha}$, and canonically conjugated momentum $\hat{\mathbf{p}}_{\alpha}$. The fundamental Bosonic fields $\hat{\mathbf{f}}_{\lambda}(\mathbf{r}, \omega)$ [and $\left.\hat{\mathbf{f}}_{\lambda}^{\dagger}(\mathbf{r}, \omega)\right]$ which can be related to noise polarization (for $\lambda$ $=e$ ) and noise magnetization (for $\lambda=m$ ), respectively, are the dynamical variables for describing the system composed of the electromagnetic field and the medium including the dissipative system responsible for absorption,

$$
\begin{aligned}
& {\left[\hat{f}_{\lambda i}(\mathbf{r}, \omega), \hat{f}_{\lambda^{\prime} i^{\prime}}^{\dagger}\left(\mathbf{r}^{\prime}, \omega^{\prime}\right)\right]=\delta_{\lambda \lambda^{\prime}} \delta_{i i^{\prime}} \delta\left(\mathbf{r}-\mathbf{r}^{\prime}\right) \delta\left(\omega-\omega^{\prime}\right),} \\
& {\left[\hat{f}_{\lambda i}(\mathbf{r}, \omega), \hat{f}_{\lambda^{\prime} i^{\prime}}\left(\mathbf{r}^{\prime}, \omega^{\prime}\right)\right]=0 .}
\end{aligned}
$$

Note that the first term on the right-hand side of Eq. (11) is the energy of that system. Further $\hat{\mathbf{A}}(\mathbf{r})$ and $\hat{\varphi}(\mathbf{r})$ are the vector and scalar potentials of the medium-assisted electromagnetic field, respectively, which in Coulomb gauge are expressed in terms of the fundamental fields $\hat{\mathbf{f}}_{\lambda}(\mathbf{r}, \omega)\left[\right.$ and $\left.\hat{\mathbf{f}}_{\lambda}^{\dagger}(\mathbf{r}, \omega)\right]$ as

$$
\begin{gathered}
\hat{\mathbf{A}}(\mathbf{r})=\int_{0}^{\infty} \mathrm{d} \omega(i \omega)^{-1} \underline{\hat{\mathbf{E}}}^{\perp}(\mathbf{r}, \omega)+\text { H.c. }, \\
-\boldsymbol{\nabla} \hat{\varphi}(\mathbf{r})=\int_{0}^{\infty} \mathrm{d} \omega \underline{\hat{\mathbf{E}}}^{\|}(\mathbf{r}, \omega)+\text { H.c., }
\end{gathered}
$$

where

$$
\hat{\hat{\mathbf{E}}}(\mathbf{r}, \omega)=\sum_{\lambda=e, m} \int \mathrm{d}^{3} r^{\prime} \boldsymbol{G}_{\lambda}\left(\mathbf{r}, \mathbf{r}^{\prime}, \omega\right) \hat{\mathbf{f}}_{\lambda}\left(\mathbf{r}^{\prime}, \omega\right),
$$




$$
\begin{aligned}
& \boldsymbol{G}_{e}\left(\mathbf{r}, \mathbf{r}^{\prime}, \omega\right)=i \frac{\omega^{2}}{c^{2}} \sqrt{\frac{\hbar}{\pi \varepsilon_{0}} \operatorname{Im} \varepsilon\left(\mathbf{r}^{\prime}, \omega\right)} \boldsymbol{G}\left(\mathbf{r}, \mathbf{r}^{\prime}, \omega\right), \\
& \boldsymbol{G}_{m}\left(\mathbf{r}, \mathbf{r}^{\prime}, \omega\right)=-i \frac{\omega}{c} \sqrt{-\frac{\hbar}{\pi \varepsilon_{0}} \operatorname{Im} \kappa\left(\mathbf{r}^{\prime}, \omega\right)} \\
& \times\left[\boldsymbol{G}\left(\mathbf{r}, \mathbf{r}^{\prime}, \omega\right) \times \overleftarrow{\nabla}_{\mathbf{r}^{\prime}}\right],
\end{aligned}
$$

with $\left[\boldsymbol{G}\left(\mathbf{r}, \mathbf{r}^{\prime}, \omega\right) \times \overleftarrow{\nabla}_{\mathbf{r}^{\prime}}\right]_{i j}=\epsilon_{j k l} \partial_{l}^{\prime} G_{i k}\left(\mathbf{r}, \mathbf{r}^{\prime}, \omega\right)$ and $\kappa(\mathbf{r}, \omega)$ $=\mu^{-1}(\mathbf{r}, \omega)$. Here and in the following, transverse and longitudinal vector fields are denoted by $\perp$ and $\|$, respectively, e.g.,

$$
\underline{\hat{\mathbf{E}}}^{\perp(\|)}(\mathbf{r}, \omega)=\int \mathrm{d}^{3} r^{\prime} \boldsymbol{\delta}^{\perp(\|)}\left(\mathbf{r}-\mathbf{r}^{\prime}\right) \underline{\hat{\mathbf{E}}}\left(\mathbf{r}^{\prime}, \omega\right),
$$

with

$$
\delta_{i j}^{\|}(\mathbf{r})=-\partial_{i} \partial_{j}\left(\frac{1}{4 \pi r}\right)
$$

and

$$
\delta_{i j}^{\perp}(\mathbf{r})=\delta(\mathbf{r}) \delta_{i j}-\delta_{i j}^{\|}(\mathbf{r})
$$

being the longitudinal and transverse dyadic $\delta$ functions, respectively.

In Eqs. (9) and (10), $\boldsymbol{G}\left(\mathbf{r}, \mathbf{r}^{\prime}, \omega\right)$ is the (classical) Green tensor, which in the case of magnetodielectric matter obeys the equation

$$
\left[\boldsymbol{\nabla} \times \kappa(\mathbf{r}, \omega) \boldsymbol{\nabla} \times-\frac{\omega^{2}}{c^{2}} \varepsilon(\mathbf{r}, \omega)\right] \boldsymbol{G}\left(\mathbf{r}, \mathbf{r}^{\prime}, \omega\right)=\boldsymbol{\delta}\left(\mathbf{r}-\mathbf{r}^{\prime}\right)
$$

together with the boundary condition

$$
\boldsymbol{G}\left(\mathbf{r}, \mathbf{r}^{\prime}, \omega\right) \rightarrow 0 \text { for }\left|\mathbf{r}-\mathbf{r}^{\prime}\right| \rightarrow \infty
$$

Note that the (relative) permittivity $\varepsilon(\mathbf{r}, \omega)$ and permeability $\mu(\mathbf{r}, \omega)$ of the (inhomogeneous) medium are complex functions of frequency, whose real and imaginary parts satisfy the Kramers-Kronig relations. Since for absorbing media we have $\operatorname{Im} \varepsilon(\mathbf{r}, \omega)>0$ and $\operatorname{Im} \mu(\mathbf{r}, \omega)>0$ $\Rightarrow \operatorname{Im} \kappa(\mathbf{r}, \omega)<0$, the expressions under the square roots in Eqs. (9) and (10) are positive. It should be pointed out that the whole space is assumed to be filled with some (absorbing) media, in which case the aforementioned conditions for $\operatorname{Im} \varepsilon(\mathbf{r}, \omega)$ and $\operatorname{Im} \mu(\mathbf{r}, \omega)$ ensure that the differential equation (14) together with the boundary condition (15) presents a well-defined problem. However, as this assumption allows for both $\varepsilon(\mathbf{r}, \omega)$ and $\mu(\mathbf{r}, \omega)$ to be arbitrarily close to unity (i.e., for arbitrarily dilute matter), it is naturally possible to include vacuum regions in the theory, by performing the limit $\varepsilon(\mathbf{r}, \omega) \rightarrow 1, \mu(\mathbf{r}, \omega)$ $\rightarrow 1$ in these regions after having calculated the desired expectation values of the relevant quantities as functions of $\varepsilon(\mathbf{r}, \omega)$ and $\mu(\mathbf{r}, \omega)$. [36]:

The Green tensor has the following useful properties

$$
\boldsymbol{G}^{*}\left(\mathbf{r}, \mathbf{r}^{\prime}, \omega\right)=\boldsymbol{G}\left(\mathbf{r}, \mathbf{r}^{\prime},-\omega^{*}\right)
$$

$$
\begin{gathered}
\boldsymbol{G}\left(\mathbf{r}, \mathbf{r}^{\prime}, \omega\right)=\boldsymbol{G}^{\top}\left(\mathbf{r}^{\prime}, \mathbf{r}, \omega\right), \\
\int \mathrm{d}^{3} s\left\{\operatorname{Im} \kappa(\mathbf{s}, \omega)\left[\boldsymbol{G}(\mathbf{r}, \mathbf{s}, \omega) \times \overleftarrow{\nabla}_{\mathbf{s}}\right]\left[\boldsymbol{\nabla}_{\mathbf{s}} \times \boldsymbol{G}^{*}\left(\mathbf{s}, \mathbf{r}^{\prime}, \omega\right)\right]\right. \\
\left.+\frac{\omega^{2}}{c^{2}} \operatorname{Im} \varepsilon(\mathbf{s}, \omega) \boldsymbol{G}(\mathbf{r}, \mathbf{s}, \omega) \boldsymbol{G}^{*}\left(\mathbf{s}, \mathbf{r}^{\prime}, \omega\right)\right\}=\operatorname{Im} \boldsymbol{G}\left(\mathbf{r}, \mathbf{r}^{\prime}, \omega\right)
\end{gathered}
$$

Combining Eq. (18) with Eqs. (9) and (10) yields

$$
\begin{gathered}
\sum_{\lambda=e, m} \int \mathrm{d}^{3} s G_{\lambda i k}(\mathbf{r}, \mathbf{s}, \omega) G_{\lambda j k}^{*}\left(\mathbf{r}^{\prime}, \mathbf{s}, \omega\right) \\
=\frac{\hbar \mu_{0}}{\pi} \omega^{2} \operatorname{Im} G_{i j}\left(\mathbf{r}, \mathbf{r}^{\prime}, \omega\right) .
\end{gathered}
$$

Note that in Eq. (19) and throughout the remaining part of this paper, summation over repeated vector indices is understood.

The total electric field is given by

$$
\hat{\vec{E}}(\mathbf{r})=\hat{\mathbf{E}}(\mathbf{r})-\nabla \hat{\varphi}_{\mathrm{A}}(\mathbf{r})
$$

where

$$
\hat{\mathbf{E}}(\mathbf{r})=\int_{0}^{\infty} \mathrm{d} \omega \underline{\hat{\mathbf{E}}}(\mathbf{r}, \omega)+\text { H.c. },
$$

with $\underline{\hat{\mathbf{E}}}(\mathbf{r}, \omega)$ from Eq. (8) . Accordingly, the total induction field reads

$$
\hat{\vec{B}}(\mathbf{r})=\hat{\mathbf{B}}(\mathbf{r})=\int_{0}^{\infty} \mathrm{d} \omega \underline{\hat{\mathbf{B}}}(\mathbf{r}, \omega)+\text { H.c. },
$$

where

$$
\underline{\hat{\mathbf{B}}}(\mathbf{r}, \omega)=(i \omega)^{-1} \nabla \times \underline{\hat{\mathbf{E}}}(\mathbf{r}, \omega) .
$$

Finally, the displacement and magnetic fields are given by

$$
\begin{aligned}
\hat{\vec{D}}(\mathbf{r}) & =\hat{\mathbf{D}}(\mathbf{r})-\varepsilon_{0} \boldsymbol{\nabla} \hat{\varphi}_{\mathrm{A}}(\mathbf{r}) \\
& =\int_{0}^{\infty} \mathrm{d} \omega[\underline{\hat{\mathbf{D}}}(\mathbf{r}, \omega)+\text { H.c. }]-\varepsilon_{0} \boldsymbol{\nabla} \hat{\varphi}_{\mathrm{A}}(\mathbf{r}), \\
\hat{\vec{H}}(\mathbf{r}) & =\hat{\mathbf{H}}(\mathbf{r})=\int_{0}^{\infty} \mathrm{d} \omega \underline{\hat{\mathbf{H}}}(\mathbf{r}, \omega)+\text { H.c. },
\end{aligned}
$$

where

$$
\begin{aligned}
\underline{\hat{\mathbf{D}}}(\mathbf{r}, \omega)= & \varepsilon_{0} \varepsilon(\mathbf{r}, \omega) \underline{\hat{\mathbf{E}}}(\mathbf{r}, \omega) \\
& +i \sqrt{\frac{\hbar \varepsilon_{0}}{\pi} \operatorname{Im} \varepsilon(\mathbf{r}, \omega) \hat{\mathbf{f}}_{e}(\mathbf{r}, \omega),} \\
\underline{\hat{\mathbf{H}}}(\mathbf{r}, \omega)= & \kappa_{0} \kappa(\mathbf{r}, \omega) \underline{\hat{\mathbf{B}}}(\mathbf{r}, \omega) \\
& -\sqrt{-\frac{\hbar \kappa_{0}}{\pi} \operatorname{Im} \kappa(\mathbf{r}, \omega)} \hat{\mathbf{f}}_{m}(\mathbf{r}, \omega) .
\end{aligned}
$$

Assuming that the atomic system is sufficiently localized, and introducing shifted particle coordinates

$$
\hat{\overline{\mathbf{r}}}_{\alpha}=\hat{\mathbf{r}}_{\alpha}-\hat{\mathbf{r}}_{\mathrm{A}}
$$


relative to the center of mass

$$
\hat{\mathbf{r}}_{\mathrm{A}}=\sum_{\alpha} \frac{m_{\alpha}}{m_{\mathrm{A}}} \hat{\mathbf{r}}_{\alpha}
$$

$\left(m_{\mathrm{A}}=\sum_{\alpha} m_{\alpha}\right)$, we can apply the long-wavelength approximation by expanding the fields $\hat{\mathbf{A}}(\mathbf{r})$ and $\hat{\varphi}(\mathbf{r})$ around the center of mass and keeping only the leading nonvanishing terms of the respective field operators. For a neutral atomic system,

$$
q_{\mathrm{A}}=\sum_{\alpha} q_{\alpha}=0
$$

this is just the familiar electric dipole approximation, and the Hamiltonian (1) simplifies to

$$
\hat{H}=\hat{H}_{\mathrm{F}}+\hat{H}_{\mathrm{A}}+\hat{H}_{\mathrm{AF}},
$$

where

$$
\begin{gathered}
\hat{H}_{\mathrm{F}} \equiv \sum_{\lambda=e, m} \int \mathrm{d}^{3} r \int_{0}^{\infty} \mathrm{d} \omega \hbar \omega \hat{\mathbf{f}}_{\lambda}^{\dagger}(\mathbf{r}, \omega) \hat{\mathbf{f}}_{\lambda}(\mathbf{r}, \omega) \\
\hat{H}_{\mathrm{A}} \equiv \sum_{\alpha} \frac{\hat{\mathbf{p}}_{\alpha}^{2}}{2 m_{\alpha}}+\frac{1}{2} \int \mathrm{d}^{3} r \hat{\rho}_{\mathrm{A}}(\mathbf{r}) \hat{\varphi}_{\mathrm{A}}(\mathbf{r}) \\
\left.\hat{H}_{\mathrm{AF}} \equiv \hat{\mathbf{d}} \boldsymbol{\nabla} \hat{\varphi}(\mathbf{r})\right|_{\mathbf{r}=\hat{\mathbf{r}}_{\mathrm{A}}}-\sum_{\alpha} \frac{q_{\alpha}}{m_{\alpha}} \hat{\mathbf{p}}_{\alpha} \hat{\mathbf{A}}\left(\hat{\mathbf{r}}_{\mathrm{A}}\right) \\
+\sum_{\alpha} \frac{q_{\alpha}^{2}}{2 m_{\alpha}} \hat{\mathbf{A}}^{2}\left(\hat{\mathbf{r}}_{\mathrm{A}}\right)
\end{gathered}
$$

with

$$
\hat{\mathbf{d}}=\sum_{\alpha} q_{\alpha} \hat{\mathbf{r}}_{\alpha}=\sum_{\alpha} q_{\alpha} \hat{\overline{\mathbf{r}}}_{\alpha}
$$

being the total electric dipole moment.

\section{B. Multipolar coupling}

Let us turn to the multipolar coupling scheme widely used for studying the interaction of electromagnetic fields with atoms and molecules. Just as in standard QED, so in the present formalism [36, 37], the multipolar Hamiltonian can be obtained from the minimal-coupling Hamiltonian by means of a Power-Zienau transformation,

$$
\hat{U}=\exp \left[\frac{i}{\hbar} \int \mathrm{d}^{3} r \hat{\mathbf{P}}_{\mathrm{A}}(\mathbf{r}) \hat{\mathbf{A}}(\mathbf{r})\right],
$$

where

$$
\hat{\mathbf{P}}_{\mathrm{A}}(\mathbf{r})=\sum_{\alpha} q_{\alpha} \hat{\overline{\mathbf{r}}}_{\alpha} \int_{0}^{1} \mathrm{~d} \lambda \delta\left(\mathbf{r}-\hat{\mathbf{r}}_{\mathrm{A}}-\lambda \hat{\overline{\mathbf{r}}}_{\alpha}\right) .
$$

For a neutral atomic system, the multipolar Hamiltonian [which is obtained by expressing the Hamiltonian (11) in terms of the transformed variables] can be given in the form of (see Appendix A)

$$
\begin{aligned}
\hat{H}= & \sum_{\lambda=e, m} \int \mathrm{d}^{3} r \int_{0}^{\infty} \mathrm{d} \omega \hbar \omega \hat{\mathbf{f}}_{\lambda}^{\prime \dagger}(\mathbf{r}, \omega) \hat{\mathbf{f}}_{\lambda}^{\prime}(\mathbf{r}, \omega) \\
& +\frac{1}{2 \varepsilon_{0}} \int \mathrm{d}^{3} r \hat{\mathbf{P}}_{\mathrm{A}}^{2}(\mathbf{r})-\int \mathrm{d}^{3} r \hat{\mathbf{P}}_{\mathrm{A}}(\mathbf{r}) \hat{\mathbf{E}}^{\prime}(\mathbf{r}) \\
& +\sum_{\alpha} \frac{1}{2 m_{\alpha}}\left[\hat{\mathbf{p}}_{\alpha}^{\prime}+\int \mathrm{d}^{3} r \hat{\boldsymbol{\Xi}}_{\alpha}(\mathbf{r}) \times \hat{\mathbf{B}}^{\prime}(\mathbf{r})\right]^{2},
\end{aligned}
$$

where

$$
\hat{\boldsymbol{\Xi}}_{\alpha}(\mathbf{r})=q_{\alpha} \hat{\boldsymbol{\Theta}}_{\alpha}(\mathbf{r})-\frac{m_{\alpha}}{m_{\mathrm{A}}} \sum_{\beta} q_{\beta} \hat{\boldsymbol{\Theta}}_{\beta}(\mathbf{r})+\frac{m_{\alpha}}{m_{\mathrm{A}}} \hat{\mathbf{P}}_{\mathrm{A}}(\mathbf{r})
$$

and

$$
\hat{\boldsymbol{\Theta}}_{\alpha}(\mathbf{r})=\hat{\overline{\mathbf{r}}}_{\alpha} \int_{0}^{1} \mathrm{~d} \lambda \lambda \delta\left(\mathbf{r}-\hat{\mathbf{r}}_{\mathrm{A}}-\lambda \hat{\overline{\mathbf{r}}}_{\alpha}\right)
$$

Note that due to the unitarity of the transformation (36), the transformed variables of the atomic system $\hat{\mathbf{r}}_{\alpha}^{\prime}=\hat{\mathbf{r}}_{\alpha}$ and $\hat{\mathbf{p}}_{\alpha}^{\prime}$ and the transformed field variables $\hat{\mathbf{f}}_{\lambda}^{\prime}(\mathbf{r}, \omega)$ and $\hat{\mathbf{f}}_{\lambda}^{\prime \dagger}(\mathbf{r}, \omega)$ obey the same commutation relations as the untransformed ones. Needless to say that the transformed fields $\hat{\mathbf{E}}^{\prime}(\mathbf{r})$ and $\hat{\mathbf{B}}^{\prime}(\mathbf{r})$ are related to the transformed fields $\hat{\mathbf{f}}_{\lambda}^{\prime}(\mathbf{r}, \omega)$ and $\hat{\mathbf{f}}_{\lambda}^{\prime \dagger}(\mathbf{r}, \omega)$ according to Eq. (8) and Eqs. (21)(23), with primed quantities instead of the unprimed ones. The Hamiltonian (38) can be regarded as the generalization of the multipolar Hamiltonian obtained earlier for moving atoms in vacuum [31, 32, 33, 34, 35] to the case where dispersing and absorbing magnetodielectric bodies are present. In particular, it can be used to describe effects specifically due to the translational motion of an atomic system such as Doppler and recoil effects.

Applying the long-wavelength approximation to the fields $\hat{\mathbf{E}}^{\prime}(\mathbf{r})$ and $\hat{\mathbf{B}}^{\prime}(\mathbf{r})$ in Eq. (38), which is equivalent to approximating $\delta\left(\mathbf{r}-\hat{\mathbf{r}}_{\mathrm{A}}-\lambda \hat{\mathbf{r}}_{\alpha}\right)$ by $\delta\left(\mathbf{r}-\hat{\mathbf{r}}_{\mathrm{A}}\right)$ in Eqs. (37) and (40), respectively, i.e.,

$$
\begin{gathered}
\hat{\mathbf{P}}_{\mathrm{A}}(\mathbf{r})=\hat{\mathbf{d}} \delta\left(\mathbf{r}-\hat{\mathbf{r}}_{\mathrm{A}}\right), \\
\hat{\boldsymbol{\Theta}}_{\alpha}(\mathbf{r})=\frac{1}{2} \hat{\overline{\mathbf{r}}}_{\alpha} \delta\left(\mathbf{r}-\hat{\mathbf{r}}_{\mathrm{A}}\right),
\end{gathered}
$$

thus

$$
\hat{\boldsymbol{\Xi}}_{\alpha}(\mathbf{r})=\frac{1}{2} q_{\alpha} \hat{\overline{\mathbf{r}}}_{\alpha} \delta\left(\mathbf{r}-\hat{\mathbf{r}}_{\mathrm{A}}\right)+\frac{m_{\alpha}}{2 m_{\mathrm{A}}} \hat{\mathbf{d}} \delta\left(\mathbf{r}-\hat{\mathbf{r}}_{\mathrm{A}}\right),
$$

we obtain the multipolar Hamiltonian in long-wavelength approximation,

$$
\hat{H}=\hat{H}_{\mathrm{F}}^{\prime}+\hat{H}_{\mathrm{A}}^{\prime}+\hat{H}_{\mathrm{AF}}^{\prime}
$$


with

$$
\begin{aligned}
\hat{H}_{\mathrm{F}}^{\prime} \equiv \sum_{\lambda=e, m} \int \mathrm{d}^{3} r \int_{0}^{\infty} \mathrm{d} \omega \hbar \omega \hat{\mathbf{f}}_{\lambda}^{\prime \dagger}(\mathbf{r}, \omega) \hat{\mathbf{f}}_{\lambda}^{\prime}(\mathbf{r}, \omega) \\
\hat{H}_{\mathrm{A}}^{\prime} \equiv \sum_{\alpha} \frac{\hat{\mathbf{p}}_{\alpha}^{\prime 2}}{2 m_{\alpha}}+\frac{1}{2 \varepsilon_{0}} \int \mathrm{d}^{3} r \hat{\mathbf{P}}_{\mathrm{A}}^{2}(\mathbf{r}) \\
\hat{H}_{\mathrm{AF}}^{\prime} \equiv-\hat{\mathbf{d}} \hat{\mathbf{E}}^{\prime}\left(\hat{\mathbf{r}}_{\mathrm{A}}\right)+\sum_{\alpha} \frac{q_{\alpha}}{2 m_{\alpha}} \hat{\overline{\mathbf{p}}}_{\alpha}^{\prime} \hat{\overline{\mathbf{r}}}_{\alpha} \times \hat{\mathbf{B}}^{\prime}\left(\hat{\mathbf{r}}_{\mathrm{A}}\right) \\
+\sum_{\alpha} \frac{q_{\alpha}^{2}}{8 m_{\alpha}}\left[\hat{\mathbf{r}}_{\alpha} \times \hat{\mathbf{B}}^{\prime}\left(\hat{\mathbf{r}}_{\mathrm{A}}\right)\right]^{2}+\frac{3}{8 m_{\mathrm{A}}}\left[\hat{\mathbf{d}} \times \hat{\mathbf{B}}^{\prime}\left(\hat{\mathbf{r}}_{\mathrm{A}}\right)\right]^{2} \\
+\frac{1}{m_{\mathrm{A}}} \hat{\mathbf{p}}_{\mathrm{A}}^{\prime} \hat{\mathbf{d}} \times \hat{\mathbf{B}}^{\prime}\left(\hat{\mathbf{r}}_{\mathrm{A}}\right),
\end{aligned}
$$

where

$$
\hat{\mathbf{p}}_{\mathrm{A}}^{\prime}=\sum_{\alpha} \hat{\mathbf{p}}_{\alpha}^{\prime}
$$

is the (canonical) momentum of the center of mass, and

$$
\hat{\overline{\mathbf{p}}}_{\alpha}^{\prime}=\hat{\mathbf{p}}_{\alpha}^{\prime}-\frac{m_{\alpha}}{m_{\mathrm{A}}} \hat{\mathbf{p}}_{\mathrm{A}}^{\prime}
$$

denote shifted momenta of the particles relative to the center of mass. The first two terms on the right-hand side of Eq. (47) represent electric and magnetic dipole interactions, respectively, the next two terms describe the (generalized) diamagnetic interaction of the charged particles with the medium-assisted electromagnetic fields, while the last term describes the Röntgen interaction due to the translational motion of the center of mass. In particular, in (generalized) electric dipole approximation, Eq. (47) reads

$$
\hat{H}_{\mathrm{AF}}^{\prime}=-\hat{\mathbf{d}} \hat{\mathbf{E}}^{\prime}\left(\hat{\mathbf{r}}_{\mathrm{A}}\right)+\frac{1}{m_{\mathrm{A}}} \hat{\mathbf{p}}_{\mathrm{A}}^{\prime} \hat{\mathbf{d}} \times \hat{\mathbf{B}}^{\prime}\left(\hat{\mathbf{r}}_{\mathrm{A}}\right) .
$$

Recall that the transformed medium-assisted electric field $\hat{\mathbf{E}}^{\prime}(\hat{\mathbf{r}})$ is related to the physical one, $\hat{\mathbf{E}}(\hat{\mathbf{r}})$, according to Eq. (A4).

If the center-of-mass coordinate is treated as a (classical) parameter $\left(\hat{\mathbf{r}}_{\mathrm{A}} \mapsto \mathbf{r}_{\mathrm{A}}\right)$, then Eq. (39) reduces to

$$
\hat{\boldsymbol{\Xi}}_{\alpha}(\mathbf{r})=q_{\alpha} \hat{\boldsymbol{\Theta}}_{\alpha}(\mathbf{r}),
$$

which corresponds to the limit $m_{\alpha} / m_{\mathrm{A}} \rightarrow 0$. Hence Eq. (47) becomes

$$
\begin{aligned}
\hat{H}_{\mathrm{AF}}^{\prime}= & -\hat{\mathbf{d}} \hat{\mathbf{E}}^{\prime}\left(\mathbf{r}_{\mathrm{A}}\right)+\sum_{\alpha} \frac{q_{\alpha}}{2 m_{\alpha}} \hat{\mathbf{p}}_{\alpha}^{\prime} \hat{\mathbf{r}}_{\alpha} \times \hat{\mathbf{B}}^{\prime}\left(\mathbf{r}_{\mathrm{A}}\right) \\
& +\sum_{\alpha} \frac{q_{\alpha}^{2}}{8 m_{\alpha}}\left[\hat{\mathbf{r}}_{\alpha} \times \hat{\mathbf{B}}^{\prime}\left(\mathbf{r}_{\mathrm{A}}\right)\right]^{2} .
\end{aligned}
$$

If the paramagnetic and diamagnetic terms are omitted, the interaction Hamiltonian simply reduces to the first term on the right-hand side of Eq. (52).

\section{VAN DER WAALS POTENTIAL}

According to Casimir's and Polder's pioneering concept [12], the CP force on an atomic system near macroscopic bodies is commonly regarded as being a conservative force. In particular, it is assumed that for an atom in an eigenstate $|l\rangle$ of the atomic Hamiltonian the positiondependent shift of the corresponding eigenvalue due to the (electric-dipole) interaction of the atomic system with the body-assisted electromagnetic field is the potential, also referred to as van der Waals (vdW) potential, from which the CP force can be derived, where the calculations are usually performed within the frame of lowest-order perturbation theory. In this picture, the center-of-mass coordinate is a parameter rather than a dynamical variable $\left(\hat{\mathbf{r}}_{\mathrm{A}} \mapsto \mathbf{r}_{\mathrm{A}}\right)$. Following this line, we first extend previous results [20], and show that minimal and multipolar coupling schemes yield essentially the same expression for the force.

\section{A. Minimal coupling}

We start from the minimal-coupling Hamiltonian in electric dipole approximation as given by Eqs. (31)-(34) together with Eq. (35) $\left(\hat{\mathbf{r}}_{\mathrm{A}} \mapsto \mathbf{r}_{\mathrm{A}}\right)$. Let $|n\rangle$ denote the eigenstates of the multilevel atomic system and write $\hat{H}_{\mathrm{A}}$ [Eq. (33)] as

$$
\hat{H}_{\mathrm{A}}=\sum_{n} E_{n}|n\rangle\langle n| .
$$

To calculate the leading-order correction to the unperturbed eigenvalue of a state $|l\rangle|\{0\}\rangle$ due to the perturbation Hamiltonian (34) $[|\{0\}\rangle$, ground state of the fundamental fields $\hat{\mathbf{f}}_{\lambda}(\mathbf{r}, \omega)$ ], we first note that the first two terms have no diagonal elements. Thus they start to contribute in second order,

$$
\begin{aligned}
\Delta_{2} E_{l}= & -\frac{1}{\hbar} \sum_{k} \sum_{\lambda=e, m} \mathcal{P} \int_{0}^{\infty} \frac{\mathrm{d} \omega}{\omega_{k l}+\omega} \int \mathrm{d}^{3} r \\
& \times \mid\langle l|\left\langle\{0\}|\hat{\mathbf{d}} \boldsymbol{\nabla} \hat{\varphi}(\mathbf{r})|_{\mathbf{r}=\mathbf{r}_{\mathrm{A}}}-\sum_{\alpha} \frac{q_{\alpha}}{m_{\alpha}} \hat{\mathbf{p}}_{\alpha} \hat{\mathbf{A}}\left(\mathbf{r}_{\mathrm{A}}\right)\right. \\
& \times\left.\left|\left\{\mathbf{1}_{\lambda}(\mathbf{r}, \omega)\right\}\right\rangle|k\rangle\right|^{2}
\end{aligned}
$$

$(\mathcal{P}$, principal part), whereas the third term starts to contribute in first order,

$$
\Delta_{1} E_{l}=\left\langle l\left|\left\langle\{0\}\left|\sum_{\alpha} \frac{q_{\alpha}^{2}}{2 m_{\alpha}} \hat{\mathbf{A}}^{2}\left(\mathbf{r}_{\mathrm{A}}\right)\right|\{0\}\right\rangle\right| l\right\rangle .
$$

Here, $\left|\left\{\mathbf{1}_{\lambda}(\mathbf{r}, \omega)\right\}\right\rangle \equiv \hat{\mathbf{f}}_{\lambda}^{\dagger}(\mathbf{r}, \omega)|\{0\}\rangle$ denotes single-quantum Fock states of the fundamental fields, and

$$
\omega_{k l} \equiv\left(E_{k}-E_{l}\right) / \hbar
$$

are the atomic transition frequencies. Since $\Delta_{1} E_{l}$ and $\Delta_{2} E_{l}$ are quadratic in the coupling constant [Eqs. (B9) 
and (B10) in Appendix $\mathrm{B}$, thus being of the same order of magnitude, the leading-order correction to the eigenvalue is given by

$$
\Delta E_{l}=\Delta_{1} E_{l}+\Delta_{2} E_{l} .
$$

A straightforward but somewhat lengthy calculation yields (see Appendix C)

$$
\begin{aligned}
\Delta E_{l}= & \frac{\mu_{0}}{\pi} \sum_{k} \mathcal{P} \int_{0}^{\infty} \frac{\mathrm{d} \omega}{\omega_{k l}+\omega} \mathbf{d}_{l k}\left\{\omega_{k l} \omega\right. \\
& \times\left[\operatorname{Im} \boldsymbol{G}\left(\mathbf{r}_{\mathrm{A}}, \mathbf{r}_{\mathrm{A}}, \omega\right)-\operatorname{Im}{ }^{\|} \boldsymbol{G}^{\|}\left(\mathbf{r}_{\mathrm{A}}, \mathbf{r}_{\mathrm{A}}, \omega\right)\right] \\
& \left.-\omega^{2} \operatorname{Im}{ }^{\|} \boldsymbol{G}^{\|}\left(\mathbf{r}_{\mathrm{A}}, \mathbf{r}_{\mathrm{A}}, \omega\right)\right\} \mathbf{d}_{k l},
\end{aligned}
$$

with

$$
\mathbf{d}_{l k}=\langle l|\hat{\mathbf{d}}| k\rangle
$$

being the dipole matrix elements.

Since the atomic system should be located in a freespace region, the Green tensor in this region is a linear superposition of the (translationally invariant) vacuum Green tensor $\boldsymbol{G}^{(0)}$ and the scattering Green tensor $\boldsymbol{G}^{(1)}$ that accounts for the spatial variation of the permittivity and permeability,

$$
\boldsymbol{G}\left(\mathbf{r}, \mathbf{r}^{\prime}, \omega\right)=\boldsymbol{G}^{(0)}\left(\mathbf{r}, \mathbf{r}^{\prime}, \omega\right)+\boldsymbol{G}^{(1)}\left(\mathbf{r}, \mathbf{r}^{\prime}, \omega\right)
$$

As a consequence, the eigenvalue correction $\Delta E_{l}$ can be decomposed into two parts,

$$
\Delta E_{l}=\Delta E_{l}^{(0)}+\Delta E_{l}^{(1)}\left(\mathbf{r}_{\mathrm{A}}\right) .
$$

The $\mathbf{r}_{\mathrm{A}}$-independent term $\Delta E_{l}^{(0)}$ associated with the vacuum Green tensor gives rise to the vacuum Lamb shift and is not of interest here. The $\mathbf{r}_{\mathrm{A}}$-dependent term $\Delta E_{l}^{(1)}\left(\mathbf{r}_{\mathrm{A}}\right)$, associated with the scattering Green tensor, is just the vdW potential sought,

$$
U_{l}\left(\mathbf{r}_{\mathrm{A}}\right)=\Delta E_{l}^{(1)}\left(\mathbf{r}_{\mathrm{A}}\right)=\Delta_{1} E_{l}^{(1)}\left(\mathbf{r}_{\mathrm{A}}\right)+\Delta_{2} E_{l}^{(1)}\left(\mathbf{r}_{\mathrm{A}}\right) .
$$

Hence from Eq. (58) $\left[\boldsymbol{G}\left(\mathbf{r}_{\mathrm{A}}, \mathbf{r}_{\mathrm{A}}, \omega\right) \mapsto \boldsymbol{G}^{(1)}\left(\mathbf{r}_{\mathrm{A}}, \mathbf{r}_{\mathrm{A}}, \omega\right)\right]$ we derive, on recalling Eq. (16) and changing the integration variable from $-\omega$ to $\omega$,

$$
\begin{aligned}
& U_{l}\left(\mathbf{r}_{\mathrm{A}}\right)=\frac{\mu_{0}}{2 i \pi} \sum_{k} \mathbf{d}_{l k}\left[\mathcal{P} \int_{0}^{\infty} \frac{\mathrm{d} \omega}{\omega_{k l}+\omega}\right. \\
& \times\left\{\omega_{k l} \omega\left[\boldsymbol{G}^{(1)}\left(\mathbf{r}_{\mathrm{A}}, \mathbf{r}_{\mathrm{A}}, \omega\right)-{ }^{\boldsymbol{G}^{(1)} \|}\left(\mathbf{r}_{\mathrm{A}}, \mathbf{r}_{\mathrm{A}}, \omega\right)\right]\right. \\
& \left.\quad-\omega^{2 \|} \boldsymbol{G}^{(1) \|}\left(\mathbf{r}_{\mathrm{A}}, \mathbf{r}_{\mathrm{A}}, \omega\right)\right\}-\mathcal{P} \int_{0}^{-\infty} \frac{\mathrm{d} \omega}{\omega_{k l}-\omega} \\
& \times\left\{\omega_{k l} \omega\left[\boldsymbol{G}^{(1)}\left(\mathbf{r}_{\mathrm{A}}, \mathbf{r}_{\mathrm{A}}, \omega\right)-{ }^{\boldsymbol{G}^{(1) \|}}\left(\mathbf{r}_{\mathrm{A}}, \mathbf{r}_{\mathrm{A}}, \omega\right)\right]\right. \\
& \left.\left.\quad+\omega^{2 \|} \boldsymbol{G}^{(1) \|}\left(\mathbf{r}_{\mathrm{A}}, \mathbf{r}_{\mathrm{A}}, \omega\right)\right\}\right] \mathbf{d}_{k l} .
\end{aligned}
$$

This equation can be greatly simplified by using contour-integral techniques. $\boldsymbol{G}^{(1)}\left(\mathbf{r}_{\mathrm{A}}, \mathbf{r}_{\mathrm{A}}, \omega\right)$ is an analytic function in the upper half of the complex frequency plane, including the real axis (apart from a possible pole at $\omega=0$ ). Furthermore, knowing the asymptotic behaviour of the Green tensor in the limit $\omega \rightarrow 0$ (cf. Ref. 37]), one can verify that all integrands in Eq. (63) remain finite in this limit. We may therefore apply Cauchy's theorem, and replace the principal value integral over the positive (negative) real half axis by a contour integral along the positive imaginary half axis (introducing the purely imaginary coordinate $\omega=i u$ ) and along a quarter circle with infinitely large radius in the first (second) quadrant of the complex frequency plane plus, in the case of $\omega_{l k}>0$, a contour integral along an infinitesimally small half circle around $\omega=\omega_{l k}\left(\omega=-\omega_{l k}\right)$ in the first (second) quadrant of the complex frequency plane. The integrals along the infinitely large quarter circles vanish due to the asymptotic property

$$
\lim _{|\omega| \rightarrow \infty} \frac{\omega^{2}}{c^{2}} \boldsymbol{G}^{(1)}(\mathbf{r}, \mathbf{r}, \omega)=0
$$

(cf. Ref. [37]), so we finally arrive at

$$
U_{l}\left(\mathbf{r}_{\mathrm{A}}\right)=U_{l}^{\mathrm{or}}\left(\mathbf{r}_{\mathrm{A}}\right)+U_{l}^{\mathrm{r}}\left(\mathbf{r}_{\mathrm{A}}\right),
$$

where

$$
\begin{aligned}
U_{l}^{\mathrm{or}}\left(\mathbf{r}_{\mathrm{A}}\right)= & \frac{\mu_{0}}{\pi} \sum_{k} \int_{0}^{\infty} \mathrm{d} u \frac{\omega_{k l} u^{2}}{\omega_{k l}^{2}+u^{2}} \\
& \times \mathbf{d}_{l k} \boldsymbol{G}^{(1)}\left(\mathbf{r}_{\mathrm{A}}, \mathbf{r}_{\mathrm{A}}, i u\right) \mathbf{d}_{k l}
\end{aligned}
$$

is the off-resonant part of the vdW potential, and

$$
\begin{aligned}
U_{l}^{\mathrm{r}}\left(\mathbf{r}_{\mathrm{A}}\right)= & -\mu_{0} \sum_{k} \Theta\left(\omega_{l k}\right) \omega_{l k}^{2} \\
& \times \mathbf{d}_{l k} \operatorname{Re} \boldsymbol{G}^{(1)}\left(\mathbf{r}_{\mathrm{A}}, \mathbf{r}_{\mathrm{A}}, \omega_{l k}\right) \mathbf{d}_{k l}
\end{aligned}
$$

$[\Theta(z)$, unit step function] is the resonant part arising from the contribution from the residua at the poles. Note that $U_{l}^{\mathrm{r}}\left(\mathbf{r}_{\mathrm{A}}\right)$ vanishes when the atomic system is in the ground state. For an atomic system in an excited state, $U_{l}^{\mathrm{r}}\left(\mathbf{r}_{\mathrm{A}}\right)$ may dominate $U_{l}^{\text {or }}\left(\mathbf{r}_{\mathrm{A}}\right)$.

The CP force can be derived from Eq. (65) according to

$$
\mathbf{F}_{l}\left(\mathbf{r}_{\mathrm{A}}\right)=-\nabla_{\mathrm{A}} U_{l}\left(\mathbf{r}_{\mathrm{A}}\right)
$$

$\left(\nabla_{\mathrm{A}} \equiv \nabla_{\mathbf{r}_{\mathrm{A}}}\right)$. A formula of the type of Eq. (65) together with Eqs. (66) and (67) was first given in Ref. 23] within the frame of LRT.

To give Eq. (66) in a more compact form, we introduce the generalized atomic polarizability tensor

$$
\begin{aligned}
\boldsymbol{\alpha}_{m n}(\omega)= & \frac{1}{\hbar} \sum_{k}\left[\frac{\mathbf{d}_{m k} \otimes \mathbf{d}_{k n}}{\tilde{\omega}_{k n}-\omega-i\left(\Gamma_{k}+\Gamma_{m}\right) / 2}\right. \\
& \left.+\frac{\mathbf{d}_{k n} \otimes \mathbf{d}_{m k}}{\tilde{\omega}_{k m}+\omega+i\left(\Gamma_{k}+\Gamma_{n}\right) / 2}\right],
\end{aligned}
$$


where $\tilde{\omega}_{k m}$ are the shifted (renormalized) transition frequencies and $\Gamma_{k}$ are the excited-state widths. Following Ref. [39], we may regard

$$
\boldsymbol{\alpha}_{l}(\omega)=\boldsymbol{\alpha}_{l l}(\omega)
$$

as being the ordinary (Kramers-Kronig-consistent) polarizability tensor of an atom in state $|l\rangle$. Hence we may rewrite Eq. (66) as

$$
U_{l}^{\mathrm{or}}\left(\mathbf{r}_{\mathrm{A}}\right)=\frac{\hbar \mu_{0}}{2 \pi} \int_{0}^{\infty} \mathrm{d} u u^{2} \operatorname{Tr}\left[\boldsymbol{\alpha}_{l}^{(0)}(i u) \boldsymbol{G}^{(1)}\left(\mathbf{r}_{\mathrm{A}}, \mathbf{r}_{\mathrm{A}}, i u\right)\right]
$$

where

$$
\boldsymbol{\alpha}_{l}^{(0)}(\omega)=\lim _{\epsilon \rightarrow 0} \frac{2}{\hbar} \sum_{k} \frac{\omega_{k l}}{\omega_{k l}^{2}-\omega^{2}-i \omega \epsilon} \mathbf{d}_{l k} \otimes \mathbf{d}_{k l}
$$

is the polarizability tensor in lowest-order perturbation theory, which can be obtained from Eq. (70) together with Eq. (69) by ignoring both the level shifts and broadenings. In particular for an atom in a spherically symmetric state, we have

$$
\boldsymbol{\alpha}_{l}^{(0)}(\omega)=\alpha_{l}^{(0)}(\omega) \boldsymbol{I}=\lim _{\epsilon \rightarrow 0} \frac{2}{3 \hbar} \sum_{k} \frac{\omega_{k l}}{\omega_{k l}^{2}-\omega^{2}-i \omega \epsilon}\left|\mathbf{d}_{l k}\right|^{2} \boldsymbol{I}
$$

(I, unit tensor), so that Eq. (71) reduces to

$$
U_{l}^{\mathrm{or}}\left(\mathbf{r}_{\mathrm{A}}\right)=\frac{\hbar \mu_{0}}{2 \pi} \int_{0}^{\infty} \mathrm{d} u u^{2} \alpha_{l}^{(0)}(i u) \operatorname{Tr} \boldsymbol{G}^{(1)}\left(\mathbf{r}_{\mathrm{A}}, \mathbf{r}_{\mathrm{A}}, i u\right)
$$

and Eq. (67) simplifies to

$$
\begin{aligned}
U_{l}^{\mathrm{r}}\left(\mathbf{r}_{\mathrm{A}}\right)= & -\frac{\mu_{0}}{3} \sum_{k} \Theta\left(\omega_{l k}\right) \omega_{l k}^{2}\left|\mathbf{d}_{l k}\right|^{2} \\
& \times \operatorname{Tr}\left[\operatorname{Re} \boldsymbol{G}^{(1)}\left(\mathbf{r}_{\mathrm{A}}, \mathbf{r}_{\mathrm{A}}, \omega_{l k}\right)\right] .
\end{aligned}
$$

Note that

$$
\boldsymbol{\alpha}_{l}(i u) \simeq \boldsymbol{\alpha}_{l}^{(0)}(i u)
$$

is typically valid for an atomic system in free space, because of the smallness of the level shifts and broadenings that result from the interaction of the atomic system with the vacuum electromagnetic field.

Equation (65) together with Eqs. (66) and (67) can be regarded as being the natural extension of the QED results obtained on the basis of the familiar normal-mode formalism, which ignores material absorption. Moreover, it does not only apply to arbitrary causal dielectric bodies, but, to our knowledge, it first proves applicable to magnetodielectric matter such as left-handed material, for which standard quantization concepts run into difficulties. Note that all information about the electric and magnetic properties of the matter is contained in the scattering Green tensor.
Finally, let us briefly comment on the ground-state potential as given by Eq. (171) for $l=0$. In terms of an integral along the positive frequency axis, it reads

$$
\begin{aligned}
& U_{0}\left(\mathbf{r}_{\mathrm{A}}\right)=-\frac{\hbar \mu_{0}}{2 \pi} \int_{0}^{\infty} \mathrm{d} \omega \omega^{2} \\
& \quad \times \operatorname{Im}\left\{\operatorname{Tr}\left[\boldsymbol{\alpha}_{0}^{(0)}(\omega) \boldsymbol{G}^{(1)}\left(\mathbf{r}_{\mathrm{A}}, \mathbf{r}_{\mathrm{A}}, \omega\right)\right]\right\} .
\end{aligned}
$$

An expression of this type can also be obtained by using the methods of LRT [23, 25]. It allows for a simple physical interpretation for the ground-state CP force as being due to correlations of the fluctuating electromagnetic field with the corresponding induced electric dipole of the atomic system plus the correlations of the fluctuating electric dipole moment with its induced electric field [28].

\section{B. Multipolar coupling}

Let us now consider the multipolar Hamiltonian in long-wavelength approximation as given by Eqs. (44)(46) together with Eq. (152), and write $\hat{H}_{\mathrm{A}}^{\prime}$ [Eq. (46)] in the form of Eq. (53). In contrast to the electric dipole approximation considered in the minimal coupling scheme, the present Hamiltonian also includes magnetic interactions. One might therefore expect that the leading-order corrections to the unperturbed eigenvalues are given by the second-order corrections due to the dipole interactions (linear in the field variables) plus the first-order correction due to the diamagnetic interaction (quadratic in the field variables), all of these contributions being quadratic in the coupling constant. However, one can show [Eqs. (B16)-(B18) in Appendix B] that the secondorder eigenvalue correction due to magnetic dipole interaction is smaller than that due to the electric dipole interaction by a factor of $\left(Z_{\text {eff }} \alpha_{0}\right)^{2}$, where $Z_{\text {eff }}$ is the effective nucleus charge felt by the electrons giving the main contribution to the energy shift, and $\alpha_{0}$ is the fine-structure constant. The current formalism based on Hamiltonian (11) only treats nonrelativistic atomic systems, which are characterized by $Z_{\text {eff }} \alpha_{0} \ll 1$ [40], so we can safely neglect the correction arising from the magnetic dipole interaction. Furthermore, the first-order correction arising from the diamagnetic term can be shown to be smaller than the second-order correction due to the electric dipole interaction by the same factor $\left(Z_{\text {eff }} \alpha_{0}\right)^{2}$, so we can disregard it for the same reason.

In summary, the main contribution to the eigenvalue shift of a state $|l\rangle\left|\left\{0^{\prime}\right\}\right\rangle\left[\left|\left\{0^{\prime}\right\}\right\rangle\right.$, ground state of the transformed fundamental fields $\left.\hat{\mathbf{f}}_{\lambda}^{\prime}(\mathbf{r}, \omega)\right]$ is the secondorder correction due to the electric dipole interaction in 
Eq. (52), i.e.,

$$
\begin{aligned}
& \Delta E_{l}=\Delta_{2} E_{l}=-\frac{1}{\hbar} \sum_{k} \sum_{\lambda=e, m} \mathcal{P} \int_{0}^{\infty} \frac{\mathrm{d} \omega}{\omega_{k l}+\omega} \\
& \quad \times \int \mathrm{d}^{3} r\left|\left\langle l\left|\left\langle\left\{0^{\prime}\right\}\left|-\hat{\mathbf{d}} \hat{\mathbf{E}}^{\prime}\left(\mathbf{r}_{\mathrm{A}}\right)\right|\left\{\mathbf{1}_{\lambda}^{\prime}(\mathbf{r}, \omega)\right\}\right\rangle\right| k\right\rangle\right|^{2}
\end{aligned}
$$

$\left[\left|\left\{\mathbf{1}_{\lambda}^{\prime}(\mathbf{r}, \omega)\right\}\right\rangle \equiv \hat{\mathbf{f}}_{\lambda}^{\prime \dagger}(\mathbf{r}, \omega)\left|\left\{0^{\prime}\right\}\right\rangle\right]$. After some algebra it can be found that (see Appendix [C)

$$
\begin{array}{r}
\Delta E_{l}=-\frac{\mu_{0}}{\pi} \sum_{k} \mathcal{P} \int_{0}^{\infty} \mathrm{d} \omega \frac{\omega^{2}}{\omega_{k l}+\omega} \\
\times \mathbf{d}_{l k} \operatorname{Im} \boldsymbol{G}\left(\mathbf{r}_{\mathrm{A}}, \mathbf{r}_{\mathrm{A}}, \omega\right) \mathbf{d}_{k l} .
\end{array}
$$

We now apply the same procedure as in Sec. IIIA below Eq. (58). Replacing the Green tensor by its scattering part and transforming the frequency integral to imaginary frequencies using contour integral techniques, we arrive at exactly the same form of the vdW potential as given in Eq. (65) together with Eqs. (66) and (67). It is worth noting that the two schemes lead to equivalent results only if in the minimal-coupling scheme the $\hat{\mathbf{A}}^{2}$ coupling term is properly taken into account.

\section{CENTER-OF-MASS MOTION AND LORENTZ FORCE}

Atomic quantities that are related to the atom-field interaction can drastically change when the atomic system comes close to a macroscopic body, the spontaneous decay thus becoming purely radiationless, with decay rates and level shifts being inversely proportional to the atomsurface separation to the third power [29]. Clearly, in this case approximations of the type (76) cannot be made in general and the perturbative approach to the calculation of the CP force becomes questionable. Moreover, when the atomic system is not in the ground state, then dynamical effects can no longer be disregarded. To go beyond perturbation theory, let us first consider the centerof-mass Newtonian equation of motion and the Lorentz force therein.

\section{A. Minimal coupling}

As has been shown [37], the Heisenberg equations of motion governed by the minimal-coupling Hamiltonian (11),

$$
\ddot{\hat{\mathbf{r}}}_{\alpha}=\left(\frac{1}{i \hbar}\right)^{2}\left[\left[\hat{\mathbf{r}}_{\alpha}, \hat{H}\right], \hat{H}\right]
$$

lead to the well-known Newtonian equations of motion for the individual charged particles,

$$
m_{\alpha} \ddot{\hat{\mathbf{r}}}_{\alpha}=q_{\alpha}\left\{\hat{\vec{E}}\left(\mathbf{r}_{\alpha}\right)+\frac{1}{2}\left[\dot{\hat{\mathbf{r}}}_{\alpha} \times \hat{\vec{B}}\left(\mathbf{r}_{\alpha}\right)-\hat{\vec{B}}\left(\mathbf{r}_{\alpha}\right) \times \dot{\hat{\mathbf{r}}}_{\alpha}\right]\right\} .
$$

Summing Eq. (81) over $\alpha$, recalling definition (29), and using Eqs. (20) and (22) together with the relationship

$$
\sum_{\alpha} q_{\alpha} \nabla_{\alpha} \hat{\varphi}_{\mathrm{A}}\left(\hat{\mathbf{r}}_{\alpha}\right)=0
$$

$\left(\nabla_{\alpha} \equiv \nabla_{\hat{\mathbf{r}}_{\alpha}}\right)$, we derive

$$
m_{\mathrm{A}} \ddot{\hat{\mathbf{r}}}_{\mathrm{A}}=\hat{\mathbf{F}}
$$

where the Lorentz force takes the form

$$
\hat{\mathbf{F}}=\int \mathrm{d}^{3} r\left[\hat{\rho}_{\mathrm{A}}(\mathbf{r}) \hat{\mathbf{E}}(\mathbf{r})+\hat{\mathbf{j}}_{\mathrm{A}}(\mathbf{r}) \times \hat{\mathbf{B}}(\mathbf{r})\right],
$$

with charge density $\hat{\rho}_{\mathrm{A}}(\mathbf{r})$ and current density $\hat{\mathbf{j}}_{\mathrm{A}}(\mathbf{r})$ being defined by Eq. (2) and

$$
\hat{\mathbf{j}}_{\mathrm{A}}(\mathbf{r})=\frac{1}{2} \sum_{\alpha} q_{\alpha}\left[\dot{\hat{\mathbf{r}}}_{\alpha} \delta\left(\mathbf{r}-\hat{\mathbf{r}}_{\alpha}\right)+\delta\left(\mathbf{r}-\hat{\mathbf{r}}_{\alpha}\right) \dot{\hat{\mathbf{r}}}_{\alpha}\right],
$$

respectively. It can be shown [31, 36, 41] that for neutral atoms the atomic charge and current densities can be expressed in terms of atomic polarization and magnetization according to

$$
\hat{\rho}_{\mathrm{A}}(\mathbf{r})=-\nabla \hat{\mathbf{P}}_{\mathrm{A}}(\mathbf{r})
$$

and

$$
\hat{\mathbf{j}}_{\mathrm{A}}(\mathbf{r})=\dot{\hat{\mathbf{P}}}_{\mathrm{A}}(\mathbf{r})+\nabla \times \hat{\mathbf{M}}_{\mathrm{A}}(\mathbf{r})+\nabla \times \hat{\mathbf{M}}_{\mathrm{R}}(\mathbf{r}),
$$

respectively, where

$$
\begin{gathered}
\hat{\mathbf{M}}_{\mathrm{A}}(\mathbf{r})=\frac{1}{2} \sum_{\alpha} q_{\alpha}\left[\hat{\boldsymbol{\Theta}}_{\alpha}(\mathbf{r}) \times \dot{\overline{\mathbf{r}}}_{\alpha}-\dot{\overline{\mathbf{r}}}_{\alpha} \times \hat{\boldsymbol{\Theta}}_{\alpha}(\mathbf{r})\right], \\
\hat{\mathbf{M}}_{\mathrm{R}}(\mathbf{r})=\frac{1}{2}\left[\hat{\mathbf{P}}_{\mathrm{A}}(\mathbf{r}) \times \dot{\hat{\mathbf{r}}}_{\mathrm{A}}-\dot{\hat{\mathbf{r}}}_{\mathrm{A}} \times \hat{\mathbf{P}}_{\mathrm{A}}(\mathbf{r})\right]
\end{gathered}
$$

with $\hat{\mathbf{P}}_{\mathrm{A}}(\mathbf{r})$ and $\hat{\boldsymbol{\Theta}}_{\alpha}(\mathbf{r})$ from Eqs. (37) and (40), respectively. Note that the last term in Eq. (87) represents the so-called Röntgen current [41, 42], which is a feature of the overall translational motion of any aggregate of charges.

Inspection of Eqs. (37), (40), (88), and (89) shows that the relations

$$
\begin{gathered}
\nabla \otimes \hat{\mathbf{P}}_{\mathrm{A}}(\mathbf{r})=-\nabla_{\mathrm{A}} \otimes \hat{\mathbf{P}}_{\mathrm{A}}(\mathbf{r}), \\
\nabla \otimes \hat{\mathbf{M}}_{\mathrm{A}(\mathrm{R})}(\mathbf{r})=-\nabla_{\mathrm{A}} \otimes \hat{\mathbf{M}}_{\mathrm{A}(\mathrm{R})}(\mathbf{r})
\end{gathered}
$$

$\left(\nabla_{\mathrm{A}} \equiv \nabla_{\mathbf{r}_{\mathrm{A}}}\right)$ are valid. We therefore may write, on recalling Maxwell's equations,

$$
\begin{gathered}
-\int \mathrm{d}^{3} r\left[\boldsymbol{\nabla} \hat{\mathbf{P}}_{\mathrm{A}}(\mathbf{r})\right] \hat{\mathbf{E}}(\mathbf{r}) \\
=\nabla_{\mathrm{A}} \int \mathrm{d}^{3} r\left[\hat{\mathbf{P}}_{\mathrm{A}}(\mathbf{r}) \hat{\mathbf{E}}(\mathbf{r})\right]+\int \mathrm{d}^{3} r \hat{\mathbf{P}}_{\mathrm{A}}(\mathbf{r}) \times \dot{\hat{\mathbf{B}}}(\mathbf{r}), \\
\int \mathrm{d}^{3} r\left[\boldsymbol{\nabla} \times \hat{\mathbf{M}}_{\mathrm{A}(\mathrm{R})}(\mathbf{r})\right] \times \hat{\mathbf{B}}(\mathbf{r}) \\
=\nabla_{\mathrm{A}} \int \mathrm{d}^{3} r\left[\hat{\mathbf{M}}_{\mathrm{A}(\mathrm{R})}(\mathbf{r}) \hat{\mathbf{B}}(\mathbf{r})\right] .
\end{gathered}
$$


Substituting Eqs. (86) and (87) into Eq. (84) and using Eqs. (92) and (931), we may equivalently express the Lorentz force as

$$
\begin{aligned}
& \hat{\mathbf{F}}=\nabla_{\mathrm{A}}\left\{\int \mathrm{d}^{3} r \hat{\mathbf{P}}_{\mathrm{A}}(\mathbf{r}) \hat{\mathbf{E}}(\mathbf{r})\right. \\
&\left.+\int \mathrm{d}^{3} r\left[\hat{\mathbf{M}}_{\mathrm{A}}(\mathbf{r})+\hat{\mathbf{M}}_{\mathrm{R}}(\mathbf{r})\right] \hat{\mathbf{B}}(\mathbf{r})\right\} \\
&+\frac{\mathrm{d}}{\mathrm{d} t} \int \mathrm{d}^{3} r \hat{\mathbf{P}}_{\mathrm{A}}(\mathbf{r}) \times \hat{\mathbf{B}}(\mathbf{r}) .
\end{aligned}
$$

In long-wavelength approximation, Eqs. (88) and (89) simplify to [recall Eqs. (41) and (42)]

$$
\begin{aligned}
\hat{\mathbf{M}}_{\mathrm{A}}(\mathbf{r})=\frac{1}{4} \sum_{\alpha} q_{\alpha}[ & \delta\left(\mathbf{r}-\hat{\mathbf{r}}_{\mathrm{A}}\right) \hat{\overline{\mathbf{r}}}_{\alpha} \times \dot{\overline{\mathbf{r}}}_{\alpha} \\
& \left.-\dot{\overline{\mathbf{r}}}_{\alpha} \times \hat{\overline{\mathbf{r}}}_{\alpha} \delta\left(\mathbf{r}-\hat{\mathbf{r}}_{\mathrm{A}}\right)\right]
\end{aligned}
$$

and

$$
\hat{\mathbf{M}}_{\mathrm{R}}(\mathbf{r})=\frac{1}{2}\left[\delta\left(\mathbf{r}-\hat{\mathbf{r}}_{\mathrm{A}}\right) \hat{\mathbf{d}} \times \dot{\hat{\mathbf{r}}}_{\mathrm{A}}-\dot{\hat{\mathbf{r}}}_{\mathrm{A}} \times \hat{\mathbf{d}} \delta\left(\mathbf{r}-\hat{\mathbf{r}}_{\mathrm{A}}\right)\right],
$$

respectively, so that the Lorentz force (94) can be written as

$$
\begin{aligned}
\hat{\mathbf{F}} & =\nabla_{\mathrm{A}}\left\{\hat{\mathbf{d}} \hat{\mathbf{E}}\left(\hat{\mathbf{r}}_{\mathrm{A}}\right)+\frac{1}{2} \sum_{\alpha} q_{\alpha} \dot{\hat{\mathbf{r}}}_{\alpha} \hat{\mathbf{B}}\left(\hat{\mathbf{r}}_{\mathrm{A}}\right) \times \hat{\mathbf{r}}_{\alpha}\right. \\
& \left.+\frac{1}{2} \dot{\mathbf{r}}_{\mathrm{A}} \hat{\mathbf{B}}\left(\hat{\mathbf{r}}_{\mathrm{A}}\right) \times \hat{\mathbf{d}}\right\}+\frac{\mathrm{d}}{\mathrm{d} t}\left[\hat{\mathbf{d}} \times \hat{\mathbf{B}}\left(\hat{\mathbf{r}}_{\mathrm{A}}\right)\right] .
\end{aligned}
$$

Further, we calculate

$$
\begin{aligned}
& \frac{\mathrm{d}}{\mathrm{d} t}\left[\hat{\mathbf{d}} \times \hat{\mathbf{B}}\left(\hat{\mathbf{r}}_{\mathrm{A}}\right)\right]=\frac{i}{\hbar}\left[\hat{H}, \hat{\mathbf{d}} \times \hat{\mathbf{B}}\left(\hat{\mathbf{r}}_{\mathrm{A}}\right)\right] \\
& =\dot{\hat{\mathbf{d}}} \times \hat{\mathbf{B}}\left(\hat{\mathbf{r}}_{\mathrm{A}}\right)+\hat{\mathbf{d}} \times\left.\dot{\hat{\mathbf{B}}}(\mathbf{r})\right|_{\mathbf{r}=\hat{\mathbf{r}}_{\mathrm{A}}} \\
& \quad+\hat{\mathbf{d}} \times \frac{1}{2}\left[\dot{\mathbf{r}}_{\mathrm{A}} \nabla_{\mathrm{A}} \otimes \hat{\mathbf{B}}\left(\hat{\mathbf{r}}_{\mathrm{A}}\right)+\hat{\mathbf{B}}\left(\hat{\mathbf{r}}_{\mathrm{A}}\right) \otimes \overleftarrow{\nabla}_{\mathrm{A}} \dot{\hat{\mathbf{r}}}_{\mathrm{A}}\right] .
\end{aligned}
$$

Comparing the different terms in Eq. (97), one can show [Eqs. (B19), (B20), and (B22)-(B24) in Appendix B that the second term in curly brackets is typically smaller than the first one by a factor of $v / c+Z_{\text {eff }} \alpha_{0}(v$, velocity of the center of mass), while the third term is smaller than the first one by a factor of $v / c$. Similarly, we find [Eqs. (B25)(B27) in Appendix B that the third term in Eq. (98) is smaller than the first two terms by a factor of $v / c$. Thus in the nonrelativistic limit considered throughout the current work [cf. Hamiltonian (10) we can set

$$
\hat{\mathbf{F}}=\left\{\nabla[\hat{\mathbf{d}} \hat{\mathbf{E}}(\mathbf{r})]+\frac{\mathrm{d}}{\mathrm{d} t}[\hat{\mathbf{d}} \times \hat{\mathbf{B}}(\mathbf{r})]\right\}_{\mathbf{r}=\hat{\mathbf{r}}_{\mathrm{A}}} .
$$

In the absense of magnetodielectric bodies, Eq. (99) reduces to earlier results derived within the multipolar coupling scheme for an atom interacting with the electromagnetic field in free space [32, 33]. However, it should be pointed out that here the electric and magnetic fields $\hat{\mathbf{E}}(\mathbf{r})$ and $\hat{\mathbf{B}}(\mathbf{r})$, respectively, are the medium-assisted fields as defined by Eqs. (21) and (22) [together with Eqs. (8) and (23)]. Thus Eq. (94) or, in electric dipole approximation, Eq. (99) determine the force acting on an atomic system in the very general case of dispersing and absorbing magnetodielectric bodies being present a result that has not yet been derived elsewhere.

\section{B. Multipolar coupling}

Using the multipolar Hamiltonian (38), we obtain, on recalling that $\hat{\mathbf{r}}_{\alpha}^{\prime}=\hat{\mathbf{r}}_{\alpha}$,

$$
m_{\alpha} \dot{\hat{\mathbf{r}}}_{\alpha}=\frac{i}{\hbar}\left[\hat{H}, m_{\alpha} \hat{\mathbf{r}}_{\alpha}\right]=\hat{\mathbf{p}}_{\alpha}^{\prime}+\int \mathrm{d}^{3} r \hat{\boldsymbol{\Xi}}_{\alpha}(\mathbf{r}) \times \hat{\mathbf{B}}^{\prime}(\mathbf{r}) .
$$

Summing Eq. (100) over $\alpha$ and taking into account Eqs. (29) and (48) yields

$$
m_{\mathrm{A}} \dot{\hat{\mathbf{r}}}_{\mathrm{A}}=\hat{\mathbf{p}}_{\mathrm{A}}^{\prime}+\int \mathrm{d}^{3} r \hat{\mathbf{P}}_{\mathrm{A}}(\mathbf{r}) \times \hat{\mathbf{B}}^{\prime}(\mathbf{r}) .
$$

Equation (101) leads to

$$
\begin{aligned}
m_{\mathrm{A}} \ddot{\hat{\mathbf{r}}_{\mathrm{A}}} & =\hat{\mathbf{F}}=\frac{i}{\hbar}\left[\hat{H}, \hat{\mathbf{p}}_{\mathrm{A}}^{\prime}+\int \mathrm{d}^{3} r \hat{\mathbf{P}}_{\mathrm{A}}(\mathbf{r}) \times \hat{\mathbf{B}}^{\prime}(\mathbf{r})\right] \\
& =\frac{i}{\hbar}\left[\hat{H}, \hat{\mathbf{p}}_{\mathrm{A}}^{\prime}\right]+\frac{\mathrm{d}}{\mathrm{d} t} \int \mathrm{d}^{3} r \hat{\mathbf{P}}_{\mathrm{A}}(\mathbf{r}) \times \hat{\mathbf{B}}^{\prime}(\mathbf{r}) .
\end{aligned}
$$

To evaluate the different contributions to the first term in Eq. (102), we first recall Eq. (90) and note that

$$
\frac{i}{\hbar}\left[\frac{1}{2 \varepsilon_{0}} \int \mathrm{d}^{3} r \hat{\mathbf{P}}_{\mathrm{A}}^{2}(\mathbf{r}), \hat{\mathbf{p}}_{\mathrm{A}}^{\prime}\right]=\frac{1}{2 \varepsilon_{0}} \int \mathrm{d}^{3} r \boldsymbol{\nabla} \hat{\mathbf{P}}_{\mathrm{A}}^{2}(\mathbf{r})=0 .
$$

Further, we derive, on recalling Eq. (100),

$$
\begin{aligned}
\frac{i}{\hbar} & {\left[\sum_{\alpha} \frac{1}{2 m_{\alpha}}\left(\hat{\mathbf{p}}_{\alpha}^{\prime}+\int \mathrm{d}^{3} r \hat{\boldsymbol{\Xi}}_{\alpha}(\mathbf{r}) \times \hat{\mathbf{B}}^{\prime}(\mathbf{r})\right)^{2}, \hat{\mathbf{p}}_{\mathrm{A}}^{\prime}\right] } \\
& =-\nabla_{\mathrm{A}} \int \mathrm{d}^{3} r \frac{1}{2} \sum_{\alpha}\left[\dot{\hat{\mathbf{r}}}_{\alpha} \times \hat{\boldsymbol{\Xi}}_{\alpha}(\mathbf{r})-\hat{\boldsymbol{\Xi}}_{\alpha}(\mathbf{r}) \times \dot{\hat{\mathbf{r}}}_{\alpha}\right] \hat{\mathbf{B}}^{\prime}(\mathbf{r}) .
\end{aligned}
$$

Substituting Eqs. (103) and (104) into Eq. (102), with $\hat{H}$ as given in Eq. (38), we eventually obtain

$$
\begin{aligned}
\hat{\mathbf{F}} & =\nabla_{\mathrm{A}}\left\{\int \mathrm{d}^{3} r \hat{\mathbf{P}}_{\mathrm{A}}(\mathbf{r}) \hat{\mathbf{E}}^{\prime}(\mathbf{r})\right. \\
& \left.+\frac{1}{2} \int \mathrm{d}^{3} r \sum_{\alpha}\left[\hat{\boldsymbol{\Xi}}_{\alpha}(\mathbf{r}) \times \dot{\mathbf{r}}_{\alpha}-\dot{\hat{\mathbf{r}}}_{\alpha} \times \hat{\mathbf{\Xi}}_{\alpha}(\mathbf{r})\right] \hat{\mathbf{B}}^{\prime}(\mathbf{r})\right\} \\
& +\frac{\mathrm{d}}{\mathrm{d} t} \int \mathrm{d}^{3} r \hat{\mathbf{P}}_{\mathrm{A}}(\mathbf{r}) \times \hat{\mathbf{B}}^{\prime}(\mathbf{r}) .
\end{aligned}
$$

It can be shown (see Appendix D) that Eq. (105) is identical to Eq. (94).

It is not difficult to see [recall Eqs. (41) and (43)] that in long-wavelength approximation Eq. (105) takes the 
form of Eq. (97), but with $\hat{\mathbf{E}}^{\prime}\left(\hat{\mathbf{r}}_{\mathrm{A}}\right)$ and $\hat{\mathbf{B}}^{\prime}\left(\hat{\mathbf{r}}_{\mathrm{A}}\right)$ in place of $\hat{\mathbf{E}}\left(\hat{\mathbf{r}}_{\mathrm{A}}\right)$ and $\hat{\mathbf{B}}\left(\hat{\mathbf{r}}_{\mathrm{A}}\right)$, respectively. The time derivative $\mathrm{d}\left[\hat{\mathbf{d}} \times \hat{\mathbf{B}}^{\prime}\left(\hat{\mathbf{r}}_{\mathrm{A}}\right)\right] / \mathrm{d} t$ can then be calculated to give an expression of the form of Eq. (98) with $\hat{\mathbf{B}}\left(\hat{\mathbf{r}}_{\mathrm{A}}\right)$ replaced by $\hat{\mathbf{B}}^{\prime}\left(\hat{\mathbf{r}}_{\mathrm{A}}\right)$. Obviously, in the nonrelativistic limit we are left with an expression similar to Eq. (99). It should be pointed out that Eqs. (97) and (99) with $\hat{\mathbf{E}}\left(\hat{\mathbf{r}}_{\mathrm{A}}\right)$ and $\hat{\mathbf{B}}\left(\hat{\mathbf{r}}_{\mathrm{A}}\right)$ replaced by $\hat{\mathbf{E}}^{\prime}\left(\hat{\mathbf{r}}_{\mathrm{A}}\right)$ and $\hat{\mathbf{B}}^{\prime}\left(\hat{\mathbf{r}}_{\mathrm{A}}\right)$, respectively, yield exactly the same force as the equations with the unprimed quantities, although the physical meaning of $\hat{\mathbf{E}}^{\prime}\left(\hat{\mathbf{r}}_{\mathrm{A}}\right)$ is different from that of $\hat{\mathbf{E}}\left(\hat{\mathbf{r}}_{\mathrm{A}}\right)$ [recall that $\hat{\mathbf{B}}^{\prime}\left(\hat{\mathbf{r}}_{\mathrm{A}}\right)$ $\left.=\hat{\mathbf{B}}\left(\hat{\mathbf{r}}_{\mathrm{A}}\right)\right]$.

It is worth noting that the results of this section can serve as an example to illustrate that the electric dipole approximation has to be employed with great care. If in electric dipole approximation the Röntgen interaction primarily related to the induction field had been disregarded and Eq. (50) without the second term on the right-hand side had been used, then in the resulting expression for the force the time-derivative term, i.e., the magnetic part of the force, would have been lost. Note that the pressure exerted by external laser fields on macroscopic bodies can be dominated by this magnetic force [43, 44], which contrasts with arguments [32, 45] that the contribution of this term to the radiation force on atoms can be neglected.

\section{AVERAGE LORENTZ FORCE}

Let us now turn to the problem of determining the electromagnetic force acting on an atomic system that is initially prepared in an arbitrary internal (electronic) quantum state. For convenience, we shall employ the multipolar formalism. On recalling Eqs. (21) and (22) together with Eq. (23), we find that Eq. (99) [with $\hat{\mathbf{E}}\left(\hat{\mathbf{r}}_{\mathrm{A}}\right)$ and $\hat{\mathbf{B}}\left(\hat{\mathbf{r}}_{\mathrm{A}}\right)$ replaced by $\hat{\mathbf{E}}^{\prime}\left(\hat{\mathbf{r}}_{\mathrm{A}}\right)$ and $\hat{\mathbf{B}}^{\prime}\left(\hat{\mathbf{r}}_{\mathrm{A}}\right)$, respectively] can be rewritten as

$$
\begin{aligned}
\hat{\mathbf{F}}=\{ & \left\{\int_{0}^{\infty} \mathrm{d} \omega \boldsymbol{\nabla}\left[\hat{\mathbf{d}} \underline{\hat{\mathbf{E}}}^{\prime}(\mathbf{r}, \omega)\right]\right. \\
& \left.+\frac{1}{i \omega} \frac{\mathrm{d}}{\mathrm{d} t} \hat{\mathbf{d}} \times\left[\boldsymbol{\nabla} \times \underline{\hat{\mathbf{E}}}^{\prime}(\mathbf{r}, \omega)\right]\right\}_{\mathbf{r}=\hat{\mathbf{r}}_{\mathrm{A}}}+\text { H.c. },
\end{aligned}
$$

where $\underline{\mathbf{E}}^{\prime}(\mathbf{r}, \omega)$ is defined according to Eq. (8). Decomposing $\hat{\mathbf{F}}$ into an average component $\langle\hat{\mathbf{F}}\rangle$ (where the expectation value $\langle\ldots\rangle$ is taken with respect to the internal atomic motion and the medium-assisted electromagnetic field only) and a fluctuating component

$$
\Delta \hat{\mathbf{F}}=\hat{\mathbf{F}}-\langle\hat{\mathbf{F}}\rangle,
$$

we may write

$$
\hat{\mathbf{F}}=\langle\hat{\mathbf{F}}\rangle+\Delta \hat{\mathbf{F}} .
$$

In the following, we will only consider the average force $\langle\hat{\mathbf{F}}\rangle$ (for a discussion of the force fluctuation $\left\langle\Delta \hat{\mathbf{F}}^{2}\right\rangle$, see, e.g., Ref. [19]). Note that we are free to choose a convenient operator ordering in Eq. (106), because $\underline{\mathbf{E}}^{\prime}(\mathbf{r}, \omega)$ commutes with $\hat{\mathbf{d}}$.

\section{A. General case}

In order to calculate the average force as a function of time, we first formally integrate the Heisenberg equations of motion for the fundamental fields $\hat{\mathbf{f}}_{\lambda}^{\prime}(\mathbf{r}, \omega, t)$ to obtain the source-quantity representation of $\underline{\mathbf{E}}^{\prime}(\mathbf{r}, \omega, t)$. The result reads (see Appendix E)

$$
\underline{\hat{\mathbf{E}}}^{\prime}(\mathbf{r}, \omega, t)=\underline{\hat{\mathbf{E}}}_{\text {free }}^{\prime}(\mathbf{r}, \omega, t)+\underline{\hat{\mathbf{E}}}_{\text {source }}^{\prime}(\mathbf{r}, \omega, t),
$$

where

$$
\underline{\hat{\mathbf{E}}}_{\text {free }}^{\prime}(\mathbf{r}, \omega, t)=\underline{\hat{\mathbf{E}}}^{\prime}(\mathbf{r}, \omega) e^{-i \omega t}
$$

and

$$
\begin{aligned}
& \underline{\mathbf{E}}_{\text {source }}^{\prime}(\mathbf{r}, \omega, t) \\
& \quad=\frac{i \mu_{0}}{\pi} \omega^{2} \int_{0}^{t} \mathrm{~d} t^{\prime} e^{-i \omega\left(t-t^{\prime}\right)} \operatorname{Im} \boldsymbol{G}\left[\mathbf{r}, \hat{\mathbf{r}}_{\mathrm{A}}\left(t^{\prime}\right), \omega\right] \hat{\mathbf{d}}\left(t^{\prime}\right) .
\end{aligned}
$$

Substituting Eq. (109) together with Eqs. (110) and (111) into Eq. (106), we arrive at

$$
\langle\hat{\mathbf{F}}(t)\rangle=\left\langle\hat{\mathbf{F}}_{\text {free }}(t)\right\rangle+\left\langle\hat{\mathbf{F}}_{\text {source }}(t)\right\rangle,
$$

where

$$
\begin{aligned}
& \left\langle\hat{\mathbf{F}}_{\text {free }}(t)\right\rangle=\left\{\int_{0}^{\infty} \mathrm{d} \omega \boldsymbol{\nabla}\left\langle\hat{\mathbf{d}}(t) \underline{\hat{\mathbf{E}}}_{\text {free }}^{\prime}(\mathbf{r}, \omega, t)\right\rangle\right. \\
& \left.\quad+\frac{1}{i \omega} \frac{\mathrm{d}}{\mathrm{d} t}\left\langle\hat{\mathbf{d}}(t) \times\left[\boldsymbol{\nabla} \times \underline{\hat{\mathbf{E}}}_{\text {free }}^{\prime}(\mathbf{r}, \omega, t)\right]\right\rangle\right\}_{\mathbf{r}=\hat{\mathbf{r}}_{\mathrm{A}}(t)}+\text { H.c. }
\end{aligned}
$$

and

$$
\left\langle\hat{\mathbf{F}}_{\text {source }}(t)\right\rangle=\left\langle\hat{\mathbf{F}}_{\text {source }}^{\text {el }}(t)\right\rangle+\left\langle\hat{\mathbf{F}}_{\text {source }}^{\mathrm{mag}}(t)\right\rangle .
$$

Here,

$$
\begin{aligned}
& \left\langle\hat{\mathbf{F}}_{\text {source }}^{\mathrm{el}}(t)\right\rangle=\left\{\frac{i \mu_{0}}{\pi} \int_{0}^{\infty} \mathrm{d} \omega \omega^{2} \int_{0}^{t} \mathrm{~d} t^{\prime} e^{-i \omega\left(t-t^{\prime}\right)}\right. \\
& \left.\times \nabla\left\langle\hat{\mathbf{d}}(t) \operatorname{Im} \boldsymbol{G}\left[\mathbf{r}, \hat{\mathbf{r}}_{\mathrm{A}}\left(t^{\prime}\right), \omega\right] \hat{\mathbf{d}}\left(t^{\prime}\right)\right\rangle\right\}_{\mathbf{r}=\hat{\mathbf{r}}_{\mathrm{A}}(t)}+\text { H.c. }
\end{aligned}
$$

is the electric part of the average force associated with the source-field part of the medium-assisted electromagnetic field, and

$$
\begin{aligned}
& \left\langle\hat{\mathbf{F}}_{\text {source }}^{\operatorname{mag}}(t)\right\rangle=\left\{\frac{\mu_{0}}{\pi} \int_{0}^{\infty} \mathrm{d} \omega \omega \frac{\mathrm{d}}{\mathrm{d} t} \int_{0}^{t} \mathrm{~d} t^{\prime} e^{-i \omega\left(t-t^{\prime}\right)}\right. \\
& \left.\times\left\langle\hat{\mathbf{d}}(t) \times\left\{\boldsymbol{\nabla} \times \operatorname{Im} \boldsymbol{G}\left[\mathbf{r}, \hat{\mathbf{r}}_{\mathrm{A}}\left(t^{\prime}\right), \omega\right]\right\} \hat{\mathbf{d}}\left(t^{\prime}\right)\right\rangle\right\}_{\mathbf{r}=\hat{\mathbf{r}}_{\mathrm{A}}(t)}+\text { H.c. }
\end{aligned}
$$


is the respective magnetic part. Equations (112)-(116) are still general in the sense that they apply to both driven and nondriven atomic systems and to both weak and strong atom-field coupling.

\section{B. Nondriven atom in the weak-coupling regime}

When the atomic system is not driven, i.e.,

$$
\left\langle\ldots \underline{\hat{\mathbf{E}}}_{\text {free }}^{\prime}\left[\hat{\mathbf{r}}_{\mathrm{A}}(t), \omega, t\right]\right\rangle=\left\langle\underline{\hat{\mathbf{E}}}_{\text {free }}^{\prime \dagger}\left[\hat{\mathbf{r}}_{\mathrm{A}}(t), \omega, t\right] \ldots\right\rangle=0
$$

then $\left\langle\hat{\mathbf{F}}_{\text {free }}(t)\right\rangle=0$. Consequently, the average force, referred to as CP force, is determined by the source-field part only,

$$
\langle\hat{\mathbf{F}}(t)\rangle=\left\langle\hat{\mathbf{F}}_{\text {source }}(t)\right\rangle .
$$

Even more specifically, we assume that the density operator of the initial quantum state of the field and the internal (electronic) motion of the atomic system reads

$$
\hat{\varrho}=\left|\left\{0^{\prime}\right\}\right\rangle\left\langle\left\{0^{\prime}\right\}\right| \otimes \hat{\sigma},
$$

where the density operator of the internal motion of the atomic system $\hat{\sigma}$ can be written as

$$
\hat{\sigma}=\sum_{m, n} \sigma_{m n} \hat{A}_{m n}
$$

$\left(\hat{A}_{m n}=|m\rangle\langle n|\right.$, with $|n\rangle,|m\rangle$ being the internal atomic energy eigenstates). In order to calculate the dipoledipole correlation function appearing in Eqs. (115) and (116), we make use of the expansion

$$
\hat{\mathbf{d}}(t)=\sum_{m, n} \mathbf{d}_{m n} \hat{A}_{m n}(t)
$$

and write

$$
\begin{aligned}
& \left\langle\hat{\mathbf{d}}(t) \otimes \hat{\mathbf{d}}\left(t^{\prime}\right)\right\rangle \\
& \quad=\sum_{m, n} \sum_{m^{\prime}, n^{\prime}} \mathbf{d}_{m n} \otimes \mathbf{d}_{m^{\prime} n^{\prime}}\left\langle\hat{A}_{m n}(t) \hat{A}_{m^{\prime} n^{\prime}}\left(t^{\prime}\right)\right\rangle .
\end{aligned}
$$

In the weak-coupling regime, the Markov approximation can be exploited and the correlation functions $\left\langle\hat{A}_{m n}(t) \hat{A}_{m^{\prime} n^{\prime}}\left(t^{\prime}\right)\right\rangle$ can be calculated by means of the quantum regression theorem (see, e.g., Ref. [46]). For this purpose, the (intra-atomic) master equation has to be solved for arbitray initial conditions, which in general requires knowledge of the specific level structure of the atomic system under consideration. Only if the relevant atomic transition frequencies are well separated from each other, one can go a step forward constructing a general solution. In this case, the off-diagonal densitymatrix elements can be regarded as being decoupled from each other and from the diagonal elements. We find (see Appendix F

$$
\begin{aligned}
& \left\langle\hat{A}_{m n}(t) \hat{A}_{m^{\prime} n^{\prime}}\left(t^{\prime}\right)\right\rangle=\delta_{n m^{\prime}}\left\langle\hat{A}_{m n^{\prime}}\left(t^{\prime}\right)\right\rangle \\
& \quad \times e^{\left\{i \tilde{\omega}_{m n}\left(\hat{\mathbf{r}}_{\mathrm{A}}\right)-\left[\Gamma_{m}\left(\hat{\mathbf{r}}_{\mathrm{A}}\right)+\Gamma_{n}\left(\hat{\mathbf{r}}_{\mathrm{A}}\right)\right] / 2\right\}\left(t-t^{\prime}\right)}
\end{aligned}
$$

$\left(t \geq t^{\prime}, m \neq n\right)$. Here,

$$
\tilde{\omega}_{m n}\left(\hat{\mathbf{r}}_{\mathrm{A}}\right)=\omega_{m n}+\delta \omega_{m}\left(\hat{\mathbf{r}}_{\mathrm{A}}\right)-\delta \omega_{n}\left(\hat{\mathbf{r}}_{\mathrm{A}}\right)
$$

are the body-induced position-dependent shifted transition frequencies $\left[\hat{\mathbf{r}}_{\mathrm{A}}=\hat{\mathbf{r}}_{\mathrm{A}}(t)\right]$, where

$$
\delta \omega_{m}\left(\hat{\mathbf{r}}_{\mathrm{A}}\right)=\sum_{k} \delta \omega_{m}^{k}\left(\hat{\mathbf{r}}_{\mathrm{A}}\right)
$$

with

$$
\delta \omega_{m}^{k}\left(\hat{\mathbf{r}}_{\mathrm{A}}\right)=\frac{\mu_{0}}{\pi \hbar} \mathcal{P} \int_{0}^{\infty} \mathrm{d} \omega \omega^{2} \frac{\mathbf{d}_{k m} \operatorname{Im} \boldsymbol{G}^{(1)}\left(\hat{\mathbf{r}}_{\mathrm{A}}, \hat{\mathbf{r}}_{\mathrm{A}}, \omega\right) \mathbf{d}_{m k}}{\tilde{\omega}_{m k}\left(\hat{\mathbf{r}}_{\mathrm{A}}\right)-\omega}
$$

and

$$
\Gamma_{m}\left(\hat{\mathbf{r}}_{\mathrm{A}}\right)=\sum_{k} \Gamma_{m}^{k}\left(\hat{\mathbf{r}}_{\mathrm{A}}\right)
$$

are the position-dependent level widths, with

$$
\begin{aligned}
\Gamma_{m}^{k}\left(\hat{\mathbf{r}}_{\mathrm{A}}\right)= & \frac{2 \mu_{0}}{\hbar} \Theta\left[\tilde{\omega}_{m k}\left(\hat{\mathbf{r}}_{\mathrm{A}}\right)\right]\left[\tilde{\omega}_{m k}\left(\hat{\mathbf{r}}_{\mathrm{A}}\right)\right]^{2} \\
& \times \mathbf{d}_{k m} \operatorname{Im} \boldsymbol{G}\left[\hat{\mathbf{r}}_{\mathrm{A}}, \hat{\mathbf{r}}_{\mathrm{A}}, \tilde{\omega}_{m k}\left(\hat{\mathbf{r}}_{\mathrm{A}}\right)\right] \mathbf{d}_{m k} .
\end{aligned}
$$

One should point out that the position-independent (infinite) Lamb-shift terms resulting from $\boldsymbol{G}^{(0)}\left(\hat{\mathbf{r}}_{\mathrm{A}}, \hat{\mathbf{r}}_{\mathrm{A}}, \omega\right)$ [recall Eq. (60)] have been thought to be absorbed in the transitions frequencies $\omega_{m n}$. Equation (126) can be rewritten by changing to imaginary frequencies [cf. the discussion below Eq. [63] ], resulting in

$$
\begin{aligned}
& \delta \omega_{m}^{k}\left(\hat{\mathbf{r}}_{\mathrm{A}}\right)=-\frac{\mu_{0}}{\hbar} \Theta\left[\tilde{\omega}_{m k}\left(\hat{\mathbf{r}}_{\mathrm{A}}\right)\right]\left[\tilde{\omega}_{m k}\left(\hat{\mathbf{r}}_{\mathrm{A}}\right)\right]^{2} \\
& \quad \times \mathbf{d}_{k m} \operatorname{Re} \boldsymbol{G}^{(1)}\left[\hat{\mathbf{r}}_{\mathrm{A}}, \hat{\mathbf{r}}_{\mathrm{A}}, \tilde{\omega}_{m k}\left(\hat{\mathbf{r}}_{\mathrm{A}}\right)\right] \mathbf{d}_{m k} \\
& +\frac{\mu_{0}}{\pi \hbar} \int_{0}^{\infty} \mathrm{d} u u^{2} \tilde{\omega}_{k m}\left(\hat{\mathbf{r}}_{\mathrm{A}}\right) \frac{\mathbf{d}_{k m} \boldsymbol{G}^{(1)}\left(\hat{\mathbf{r}}_{\mathrm{A}}, \hat{\mathbf{r}}_{\mathrm{A}}, i u\right) \mathbf{d}_{m k}}{\left[\tilde{\omega}_{k m}\left(\hat{\mathbf{r}}_{\mathrm{A}}\right)\right]^{2}+u^{2}} .
\end{aligned}
$$

Recall that in the perturbative treatment the vdW potential of an atomic system in a state $|m\rangle$ is identified with the energy shift $\hbar \delta \omega_{m}$, so it is not surprising that Eq. (125) together with Eq. (129) corresponds to Eq. (65) together with Eqs. (66) and (67), if in Eq. (129) the $\tilde{\omega}_{m k}$ are replaced with $\omega_{m k}$. The calculation of

$$
\left\langle\hat{A}_{m n}(t)\right\rangle=\sigma_{n m}(t)
$$

$\left[\sigma_{n m}(0)=\sigma_{n m}\right]$ then leads (under the assumptions made) to

$$
\sigma_{n m}(t)=e^{\left\{i \tilde{\omega}_{m n}\left(\hat{\mathbf{r}}_{\mathrm{A}}\right)-\left[\Gamma_{m}\left(\hat{\mathbf{r}}_{\mathrm{A}}\right)+\Gamma_{n}\left(\hat{\mathbf{r}}_{\mathrm{A}}\right)\right] / 2\right\} t} \sigma_{n m}
$$

for $m \neq n$ [cf. Eq. (123)], so the remaining task consists in solving the balance equations

$$
\dot{\sigma}_{m m}(t)=-\Gamma_{m}\left(\hat{\mathbf{r}}_{\mathrm{A}}\right) \sigma_{m m}(t)+\sum_{n} \Gamma_{n}^{m}\left(\hat{\mathbf{r}}_{\mathrm{A}}\right) \sigma_{n n}(t) .
$$

With these preparations at hand, the CP force can be calculated in the following steps. We first substitute Eq. (122) together with Eqs. (123) and (130) into 
Eqs. (115) and (116) and perform the time derivative in Eq. (116). Introducing slowly varying density-matrix elements $\tilde{\sigma}_{n m}(t)=e^{i \tilde{\omega}_{n m} t} \sigma_{n m}(t)$, we then perform the time integrals in the spirit of the Markov approximation, by making the replacements $\tilde{\sigma}_{n m}\left(t^{\prime}\right) \mapsto \tilde{\sigma}_{n m}(t)$ as well as $\hat{\mathbf{r}}_{\mathrm{A}}\left(t^{\prime}\right) \mapsto \hat{\mathbf{r}}_{\mathrm{A}}(t)$ and letting the upper limit of integration tend to infinity. Recalling Eq. (118) together with Eq. (114), we derive

$$
\begin{gathered}
\langle\hat{\mathbf{F}}(t)\rangle=\sum_{m, n} \sigma_{n m}(t) \mathbf{F}_{m n}\left(\hat{\mathbf{r}}_{\mathrm{A}}\right), \\
\mathbf{F}_{m n}\left(\hat{\mathbf{r}}_{\mathrm{A}}\right)=\mathbf{F}_{m n}^{\mathrm{el}}\left(\hat{\mathbf{r}}_{\mathrm{A}}\right)+\mathbf{F}_{m n}^{\operatorname{mag}}\left(\hat{\mathbf{r}}_{\mathrm{A}}\right),
\end{gathered}
$$

where

$$
\begin{aligned}
& \mathbf{F}_{m n}^{\mathrm{el}}\left(\hat{\mathbf{r}}_{\mathrm{A}}\right)=\left\{\frac{\mu_{0}}{\pi} \sum_{k} \int_{0}^{\infty} \mathrm{d} \omega \omega^{2}\right. \\
& \left.\times \frac{\nabla \otimes \mathbf{d}_{m k} \operatorname{Im} \boldsymbol{G}^{(1)}\left(\mathbf{r}, \hat{\mathbf{r}}_{\mathrm{A}}, \omega\right) \mathbf{d}_{k n}}{\omega+\tilde{\omega}_{k n}\left(\hat{\mathbf{r}}_{\mathrm{A}}\right)-i\left[\Gamma_{k}\left(\hat{\mathbf{r}}_{\mathrm{A}}\right)+\Gamma_{m}\left(\hat{\mathbf{r}}_{\mathrm{A}}\right)\right] / 2}\right\}_{\mathbf{r}=\hat{\mathbf{r}}_{\mathrm{A}}}+\text { H.c. },
\end{aligned}
$$

and

$$
\begin{aligned}
& \mathbf{F}_{m n}^{\operatorname{mag}}\left(\hat{\mathbf{r}}_{\mathrm{A}}\right)=\left\{\frac{\mu_{0}}{\pi} \sum_{k} \int_{0}^{\infty} \mathrm{d} \omega \omega \tilde{\omega}_{m n}\left(\hat{\mathbf{r}}_{\mathrm{A}}\right)\right. \\
&\left.\times \frac{\mathbf{d}_{m k} \times\left[\boldsymbol{\nabla} \times \operatorname{Im} \boldsymbol{G}^{(1)}\left(\mathbf{r}, \hat{\mathbf{r}}_{\mathrm{A}}, \omega\right)\right] \mathbf{d}_{k n}}{\omega+\tilde{\omega}_{k n}\left(\hat{\mathbf{r}}_{\mathrm{A}}\right)-i\left[\Gamma_{k}\left(\hat{\mathbf{r}}_{\mathrm{A}}\right)+\Gamma_{m}\left(\hat{\mathbf{r}}_{\mathrm{A}}\right)\right] / 2}\right\}_{\mathbf{r}=\hat{\mathbf{r}}_{\mathrm{A}}}+\text { H.c. }
\end{aligned}
$$

This result requires two comments. First, in Eqs. (135) and (136) the replacement $\boldsymbol{G}\left(\mathbf{r}, \hat{\mathbf{r}}_{\mathrm{A}}, \omega\right) \mapsto \boldsymbol{G}^{(1)}\left(\mathbf{r}, \hat{\mathbf{r}}_{\mathrm{A}}, \omega\right)$ has again been made, which can be justified by similar arguments as in Sec. III] [cf. the discussion preceding Eq. (62)]. Second, from the derivation of Eqs. (133)-(136) it is clear that these equations are valid provided that the center-of-mass motion can be regarded as being sufficiently slow. More precisely, they hold if the condition

$$
\boldsymbol{G}\left[\mathbf{r}, \hat{\mathbf{r}}_{\mathrm{A}}(t+\Delta t), \omega\right] \approx \boldsymbol{G}\left[\mathbf{r}, \hat{\mathbf{r}}_{\mathrm{A}}(t), \omega\right] \text { for } \Delta t \leq \Gamma_{\mathrm{C}}^{-1}
$$

is satisfied, where $\Gamma_{\mathrm{C}}$ is a characteristic intra-atomic decay rate. Under this condition, the internal (electronic) and external (center-of-mass) motion of the atomic system decouple in the spirit of a Born-Oppenheimer approximation. As a result, $\hat{\mathbf{r}}_{\mathrm{A}}$ effectively enters the equations as a parameter, so that the caret will be removed in the following $\left(\hat{\mathbf{r}}_{\mathrm{A}} \mapsto \mathbf{r}_{\mathrm{A}}\right)$.

We finally rewrite Eqs. (135) and (136), by using contour integration and going over to imaginary frequencies [cf. the discussion below Eq. (63)]. Recalling the definition of $\boldsymbol{\alpha}_{m n}(\omega)=\boldsymbol{\alpha}_{m n}\left(\mathbf{r}_{\mathrm{A}}, \omega\right)$ as given in Eq. (69) and introducing the abbreviating notation

$$
\Omega_{m n k}\left(\mathbf{r}_{\mathrm{A}}\right)=\tilde{\omega}_{n k}\left(\mathbf{r}_{\mathrm{A}}\right)+i\left[\Gamma_{m}\left(\mathbf{r}_{\mathrm{A}}\right)+\Gamma_{k}\left(\mathbf{r}_{\mathrm{A}}\right)\right] / 2,
$$
we derive

$$
\begin{aligned}
\mathbf{F}_{m n}^{\mathrm{el}}\left(\mathbf{r}_{\mathrm{A}}\right) & =\mathbf{F}_{m n}^{\mathrm{el}, \mathrm{or}}\left(\mathbf{r}_{\mathrm{A}}\right)+\mathbf{F}_{m n}^{\mathrm{el}, \mathrm{r}}\left(\mathbf{r}_{\mathrm{A}}\right) \\
\mathbf{F}_{m n}^{\operatorname{mag}}\left(\mathbf{r}_{\mathrm{A}}\right) & =\mathbf{F}_{m n}^{\operatorname{mag}, \mathrm{or}}\left(\mathbf{r}_{\mathrm{A}}\right)+\mathbf{F}_{m n}^{\operatorname{mag}, \mathrm{r}}\left(\mathbf{r}_{\mathrm{A}}\right),
\end{aligned}
$$

where

$$
\begin{gathered}
\mathbf{F}_{m n}^{\mathrm{el}, \mathrm{or}}\left(\mathbf{r}_{\mathrm{A}}\right)=-\left\{\frac{\hbar \mu_{0}}{2 \pi} \int_{0}^{\infty} \mathrm{d} u u^{2}\left[\left(\alpha_{m n}\right)_{i j}\left(\mathbf{r}_{\mathrm{A}}, i u\right)+\left(\alpha_{m n}\right)_{i j}\left(\mathbf{r}_{\mathrm{A}},-i u\right)\right] \nabla G_{i j}^{(1)}\left(\mathbf{r}, \mathbf{r}_{\mathrm{A}}, i u\right)\right\}_{\mathbf{r}=\mathbf{r}_{\mathrm{A}}} \\
\mathbf{F}_{m n}^{\mathrm{el}, \mathrm{r}}\left(\mathbf{r}_{\mathrm{A}}\right)=\left\{\mu_{0} \sum_{k} \Theta\left(\tilde{\omega}_{n k}\right) \Omega_{m n k}^{2}\left(\mathbf{r}_{\mathrm{A}}\right) \boldsymbol{\nabla} \otimes \mathbf{d}_{m k} \boldsymbol{G}^{(1)}\left[\mathbf{r}, \mathbf{r}_{\mathrm{A}}, \Omega_{m n k}\left(\mathbf{r}_{\mathrm{A}}\right)\right] \mathbf{d}_{k n}\right\}_{\mathbf{r}=\mathbf{r}_{\mathrm{A}}}+\text { H.c. }
\end{gathered}
$$

and

$$
\begin{aligned}
& \mathbf{F}_{m n}^{\text {mag,or }}\left(\mathbf{r}_{\mathrm{A}}\right)=\left\{\frac{\hbar \mu_{0}}{2 \pi} \int_{0}^{\infty} \mathrm{d} u u^{2} \operatorname{Tr}\left(\left[\frac{\tilde{\omega}_{m n}\left(\mathbf{r}_{\mathrm{A}}\right)}{i u} \boldsymbol{\alpha}_{m n}^{\top}\left(\mathbf{r}_{\mathrm{A}}, i u\right)-\frac{\tilde{\omega}_{m n}\left(\mathbf{r}_{\mathrm{A}}\right)}{i u} \boldsymbol{\alpha}_{m n}^{\top}\left(\mathbf{r}_{\mathrm{A}},-i u\right)\right] \times\left[\boldsymbol{\nabla} \times \boldsymbol{G}^{(1)}\left(\mathbf{r}, \mathbf{r}_{\mathrm{A}}, i u\right)\right]\right)\right\}_{\mathbf{r}=\mathbf{r}_{\mathrm{A}}}, \\
& \mathbf{F}_{m n}^{\mathrm{mag}, \mathrm{r}}\left(\mathbf{r}_{\mathrm{A}}\right)=\left\{\mu_{0} \sum_{k} \Theta\left(\tilde{\omega}_{n k}\right) \tilde{\omega}_{m n}\left(\mathbf{r}_{\mathrm{A}}\right) \Omega_{m n k}\left(\mathbf{r}_{\mathrm{A}}\right) \mathbf{d}_{m k} \times\left(\boldsymbol{\nabla} \times \boldsymbol{G}^{(1)}\left[\mathbf{r}, \mathbf{r}_{\mathrm{A}}, \Omega_{m n k}\left(\mathbf{r}_{\mathrm{A}}\right)\right] \mathbf{d}_{k n}\right)\right\}_{\mathbf{r}=\mathbf{r}_{\mathrm{A}}}+\mathrm{H} . \mathrm{c} .
\end{aligned}
$$


Eq. (68) together with Eqs. (65), (67), and (71). The above result is the first nonperturbative expression for the $\mathrm{CP}$ force that incorporates its time dependence in case of excited atoms and correctly accounts for bodyinduced shifting and broadening of atomic transition lines.

In the short-time limit, $\Gamma_{C} t \ll 1$, Eq. (133) reads

$$
\langle\hat{\mathbf{F}}(t)\rangle \simeq\langle\hat{\mathbf{F}}(0)\rangle=\sum_{m, n} \sigma_{n m}(0) \mathbf{F}_{m n}\left(\mathbf{r}_{\mathrm{A}}\right),
$$

which for $\sigma_{n m}(0)=\delta_{n l} \delta_{m l}$ reduces to

$$
\langle\hat{\mathbf{F}}(t)\rangle \simeq\langle\hat{\mathbf{F}}(0)\rangle=\mathbf{F}_{l l}^{\mathrm{el}}\left(\mathbf{r}_{\mathrm{A}}\right) .
$$

For the nonrelativistic Hamiltonian (46), we can always choose real dipole matrix elements $\left(\mathbf{d}_{m n}=\mathbf{d}_{n m}\right)$, revealing that $\mathbf{d}_{m n} \otimes \mathbf{d}_{n m}$ is a symmetric tensor so that, recalling Eq. (17), we may exploit the rule

$$
S_{i j} \nabla G_{i j}^{(1)}(\mathbf{r}, \mathbf{r}, \omega)=\left.2 S_{i j} \nabla_{\mathbf{s}} G_{i j}^{(1)}(\mathbf{s}, \mathbf{r}, \omega)\right|_{\mathbf{s}=\mathbf{r}},
$$

which is valid for any symmetric tensor $\boldsymbol{S}$. Hence, Eqs. (141) and (142) [together with Eq. (70)] lead to

$$
\begin{gathered}
\mathbf{F}_{l l}^{\mathrm{el}, \mathrm{or}}\left(\mathbf{r}_{\mathrm{A}}\right)=-\frac{\hbar \mu_{0}}{4 \pi} \int_{0}^{\infty} \mathrm{d} u u^{2}\left[\left(\alpha_{l}\right)_{i j}\left(\mathbf{r}_{\mathrm{A}}, i u\right)\right. \\
\left.+\left(\alpha_{l}\right)_{i j}\left(\mathbf{r}_{\mathrm{A}},-i u\right)\right] \nabla_{\mathrm{A}} G_{i j}^{(1)}\left(\mathbf{r}_{\mathrm{A}}, \mathbf{r}_{\mathrm{A}}, i u\right)
\end{gathered}
$$

and

$$
\begin{aligned}
& \mathbf{F}_{l l}^{\mathrm{el}, \mathrm{r}}\left(\mathbf{r}_{\mathrm{A}}\right)=\frac{\mu_{0}}{2} \sum_{k} \Theta\left(\tilde{\omega}_{l k}\right) \Omega_{l k}^{2}\left(\mathbf{r}_{\mathrm{A}}\right)\{\boldsymbol{\nabla} \\
& \left.\otimes \mathbf{d}_{l k} \boldsymbol{G}^{(1)}\left[\mathbf{r}, \mathbf{r}, \Omega_{l k}\left(\mathbf{r}_{\mathrm{A}}\right)\right] \mathbf{d}_{k l}\right\}_{\mathbf{r}=\mathbf{r}_{\mathrm{A}}}+\text { H.c. }
\end{aligned}
$$

$\left[\Omega_{l k}\left(\mathbf{r}_{\mathrm{A}}\right) \equiv \Omega_{l l k}\left(\mathbf{r}_{\mathrm{A}}\right)\right]$. Ignoring the position-dependent shifts and broadenings of the atomic energy levels, i.e., disregarding the position dependence of the atomic polarizability $\left[\boldsymbol{\alpha}_{l}\left(\mathbf{r}_{\mathrm{A}}, i u\right) \mapsto \boldsymbol{\alpha}_{l}^{(0)}(i u)\right]$, Eqs. (148) and (149) reduce to the perturbative result in Eq. (68) together with Eqs. (65), (67), and (71) $\left[\mathbf{F}_{l l}^{\mathrm{el}}\left(\mathbf{r}_{\mathrm{A}}\right) \mapsto \mathbf{F}_{l}\left(\mathbf{r}_{\mathrm{A}}\right)\right]$. Note that this result can be obtained without choosing real dipole matrix elements $\left[\boldsymbol{\alpha}_{l}(i u)+\boldsymbol{\alpha}_{l}(-i u)\right.$ being symmetric in this case]. In the long-time limit, $\Gamma_{C} t \gg 1$, Eq. (133) obviously reduces to ground-state force

$$
\langle\hat{\mathbf{F}}(t)\rangle \simeq \sum_{m, n} \sigma_{n m}(\infty) \mathbf{F}_{m n}\left(\mathbf{r}_{\mathrm{A}}\right)=\mathbf{F}_{00}^{\mathrm{el}, \mathrm{or}}\left(\mathbf{r}_{\mathrm{A}}\right)
$$

$\left[\mathbf{F}_{00}^{\mathrm{el}, \mathrm{r}}\left(\mathbf{r}_{\mathrm{A}}\right)=0\right]$, because of $\sigma_{n m}(\infty)=\delta_{n 0} \delta_{m 0}$.

As already mentioned, the expression for the groundstate CP force $\mathbf{F}_{00}\left(\mathbf{r}_{\mathrm{A}}\right)$ obtained in lowest-order perturbation theory, Eq. (77), agrees with the expression obtained from LRT. However, its naive extrapolation in the sense of the replacement $\boldsymbol{\alpha}_{0}^{(0)}(\omega) \mapsto \boldsymbol{\alpha}_{0}\left(\mathbf{r}_{\mathrm{A}}, \omega\right)$ in Eq. (77) [25] is wrong, because it results in Eq. (148) with $2\left(\alpha_{0}\right)_{i j}\left(\mathbf{r}_{\mathrm{A}}, i u\right)$ instead of $\left(\alpha_{0}\right)_{i j}\left(\mathbf{r}_{\mathrm{A}}, i u\right)+\left(\alpha_{0}\right)_{i j}\left(\mathbf{r}_{\mathrm{A}},-i u\right)$. As a result, a noticeable influence of the level broadening on the off-resonant part of the CP force is erroneously predicted in Ref. 25] (cf. Sec. VC), thus demonstrating that body-induced level broadening is a nonperturbative effect which lies beyond the scope of the LRT approach to the problem.

Equation (148) reveals that even the ground-state CP force cannot be derived from a potential in the usual way, because of the position dependence of the atomic polarizability. Nevertheless, it is a potential force, provided that it is an irrotational vector, i.e.,

$$
\begin{aligned}
\nabla_{\mathrm{A}} & \times \mathbf{F}_{00}\left(\mathbf{r}_{\mathrm{A}}\right)=\int_{0}^{\infty} \mathrm{d} u u^{2} \sum_{k}\left\{\left[\nabla_{\mathrm{A}} \tilde{\omega}_{k 0}\left(\mathbf{r}_{\mathrm{A}}\right)\right] \frac{\partial}{\partial \tilde{\omega}_{k 0}}\right. \\
+ & {\left.\left[\nabla_{\mathrm{A}} \Gamma_{k}\left(\mathbf{r}_{\mathrm{A}}\right)\right] \frac{\partial}{\partial \Gamma_{k}}\right\}\left[\left(\alpha_{0}\right)_{i j}\left(\mathbf{r}_{\mathrm{A}}, i u\right)\right.} \\
+ & \left.\left(\alpha_{0}\right)_{i j}\left(\mathbf{r}_{\mathrm{A}},-i u\right)\right] \times \nabla_{\mathrm{A}} G_{i j}^{(1)}\left(\mathbf{r}_{\mathrm{A}}, \mathbf{r}_{\mathrm{A}}, i u\right)=0 .
\end{aligned}
$$

While for effectively one-dimensional problems (e.g., for an atom in the presence of planarly, spherically, or cylindrically multilayered media) this condition is satisfied, there are of course situations where it is violated, implying that Eq. (148) is inaccessible to perturbative methods in principle.

When the atomic system is initially prepared in a coherent superposition of states such that $\sigma_{n m}(0) \neq 0$ is valid for certain values $n$ and $m$ with $n \neq m$, then according to Eq. (133) - the corresponding off-diagonal force components $\sigma_{n m}(t) \mathbf{F}_{m n}\left(\mathbf{r}_{\mathrm{A}}\right)$ can also contribute to the total force acting on the atomic system. Interestingly, such transient off-diagonal force components contain contributions not only from the electric part of the Lorentz force but also from the magnetic part, as can be easily seen from inspection of Eqs. (143) and (144). Thus an atomic qubit $|\psi\rangle=(|0\rangle+|1\rangle) / \sqrt{2}$ (cf., e.g., Ref. [47]) near a body feels, in electric dipole approximation, both an electric and a magnetic force in general.

Let us briefly comment on atomic systems displaying (quasi)degeneracies, i.e., systems exhibiting transitions with $\omega_{m n} \simeq \omega_{m^{\prime} n^{\prime}}\left(m \neq m^{\prime}\right.$ and/or $\left.n \neq n^{\prime}\right)$. In such a case, the assumption that the (relevant) off-diagonal densitymatrix elements decouple from each other as well as from the diagonal ones can no longer be made. Let us assume that the degenerate sublevels are not connected via electric dipole transitions $\left(\mathbf{d}_{m m^{\prime}}=0\right.$ if $\left.\omega_{m m^{\prime}} \simeq 0\right)$. The degeneracy related to the different possible projections of the angular momentum of an atom (in free space) onto a chosen direction is a typical example. Taking into account that the degeneracy is removed when the atom is close to a body, it may be advantageous to change the basis within each degenerate sublevel accordingly and consider the master equation in the new basis. An equation of the form of Eq. (123) is then valid in the new basis. Note that the new basis will in general depend on the position of the atom, thus introducing an additional position dependence of the CP force. While Eq. (131) also remains valid in the new basis for $\omega_{m n} \neq 0$, this is not in general true for the temporal evolution of the density-matrix elements 
with $\omega_{m m^{\prime}} \simeq 0$ so that, instead of the balance equations (132), a system of equations has to be solved in which diagonal density-matrix elements and off-diagonal elements with $\omega_{m m^{\prime}} \simeq 0$ are coupled to each other.

\section{Example: Excited atom near an interface}

To illustrate the effects of body-induced level shifting and broadening, let us consider a two-level atom with (real) transition dipole matrix element $\mathbf{d}_{\mathrm{A}} \equiv \mathbf{d}_{10}=$ $d_{\mathrm{A}}\left(\cos \phi \sin \theta \mathbf{e}_{x}+\sin \phi \sin \theta \mathbf{e}_{y}+\cos \theta \mathbf{e}_{z}\right)\left(\mathbf{d}_{00}=\mathbf{d}_{11}=0\right)$, which is situated at position $z_{\mathrm{A}}$ very close above $(z>0)$ a semi-infinite half space $(z<0)$ containing a homogeneous dispersing and absorbing magnetodielectric medium. Let $\delta \omega=\delta \omega_{1}-\delta \omega_{0}$ denote the (position-dependent) shift of the transition frequency. Using the Green tensor in the short-distance limit, from Eqs. (124), (125), and (129) we derive (see Appendix G)

$$
\begin{gathered}
\delta \omega\left(z_{\mathrm{A}}\right)=\delta \omega_{\mathrm{r}}\left(z_{\mathrm{A}}\right)+\delta \omega_{\mathrm{or}}\left(z_{\mathrm{A}}\right), \\
\delta \omega_{\mathrm{r}}\left(z_{\mathrm{A}}\right)=-\frac{C}{\hbar z_{\mathrm{A}}^{3}} \frac{\left|\varepsilon\left[\tilde{\omega}_{10}\left(z_{\mathrm{A}}\right)\right]\right|^{2}-1}{\left|\varepsilon\left[\tilde{\omega}_{10}\left(z_{\mathrm{A}}\right)\right]+1\right|^{2}}, \\
\delta \omega_{\mathrm{or}}\left(z_{\mathrm{A}}\right)=\frac{2 C \tilde{\omega}_{10}\left(z_{\mathrm{A}}\right)}{\hbar \pi z_{\mathrm{A}}^{3}} \int_{0}^{\infty} \frac{\mathrm{d} u}{\tilde{\omega}_{10}^{2}\left(z_{\mathrm{A}}\right)+u^{2}} \frac{\varepsilon(i u)-1}{\varepsilon(i u)+1},
\end{gathered}
$$

where

$$
C=\frac{d_{\mathrm{A}}^{2}\left(1+\cos ^{2} \theta\right)}{32 \pi \varepsilon_{0}} .
$$

Note that in the short-distance limit the medium effectively acts like a dielectric one. Since the relation $\tilde{\omega}_{10}=$ $\omega_{10}+\delta \omega$ is valid, Eq. (152) together with Eqs. (153) and (154) is a highly transcendental equation for the determination of $\delta \omega$. To solve it, we first note that the offresonant term $\delta \omega_{\text {or }}$ may be neglected in most practical situations. For example, for a single-resonance medium of Drude-Lorentz type,

$$
\varepsilon(\omega)=1+\frac{\omega_{\mathrm{P}}^{2}}{\omega_{\mathrm{T}}^{2}-\omega^{2}-i \gamma \omega},
$$

and the parameter values in Fig. 1, one can easily verify the inequality

$$
\frac{\delta \omega_{\text {or }}\left(z_{\mathrm{A}}\right)}{\tilde{\omega}_{10}\left(z_{\mathrm{A}}\right)} \leq \frac{C \omega_{\mathrm{P}}^{2}}{2 \hbar z_{\mathrm{A}}^{3} \omega_{\mathrm{T}}^{2} \tilde{\omega}_{10}\left(z_{\mathrm{A}}\right)} \lesssim 10^{-4} .
$$

Thus, keeping only the resonant part of the frequency shift, we may set

$$
\delta \omega\left(z_{\mathrm{A}}\right)=-\frac{C}{\hbar z_{\mathrm{A}}^{3}} \frac{\left|\varepsilon\left[\tilde{\omega}_{10}\left(z_{\mathrm{A}}\right)\right]\right|^{2}-1}{\left|\varepsilon\left[\tilde{\omega}_{10}\left(z_{\mathrm{A}}\right)\right]+1\right|^{2}} .
$$

For $\varepsilon\left(\tilde{\omega}_{10}\right)$ from Eq. (156), Eq. (158) is a fifth-order poly-
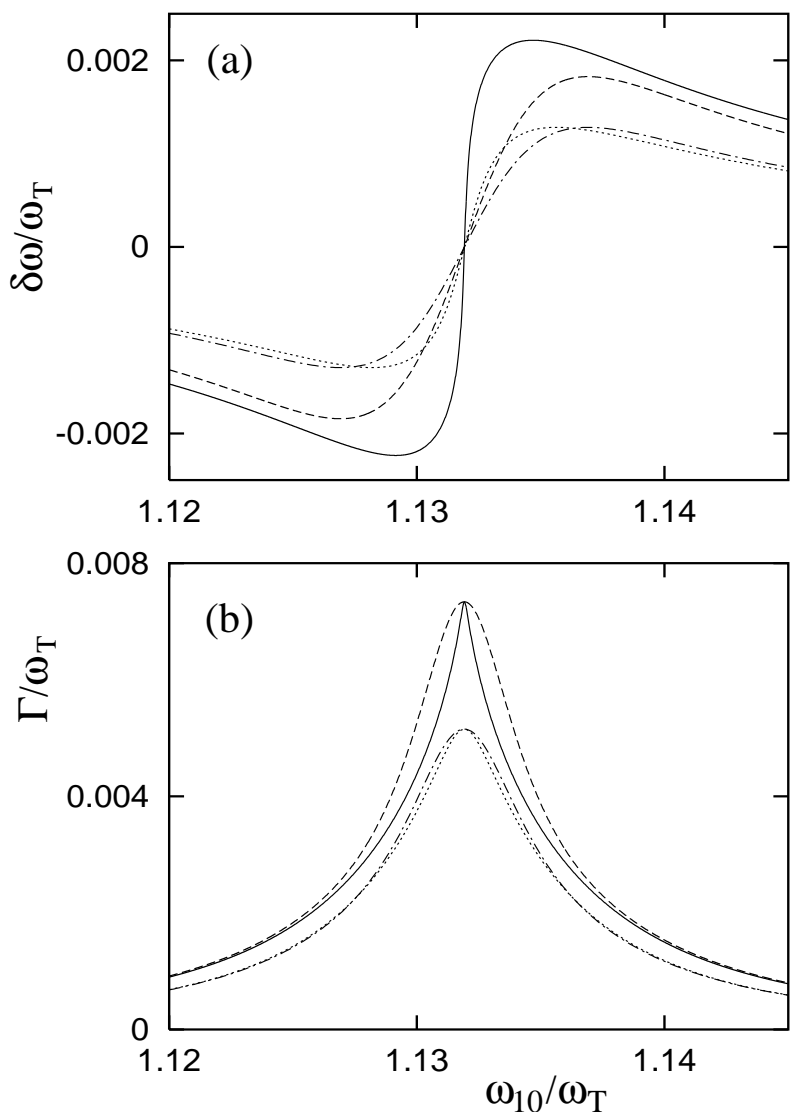

FIG. 1: (a) Transition frequency shift (solid and dotted lines) and (b) decay rate (solid and dotted lines) versus bare transition frequency for a two-level atom that is situated at distance $z_{\mathrm{A}}$ from a semi-infinite half space medium of complex permittivity according to Eq. (156) and whose transition dipole moment is perpendicular to the interface $\left[\omega_{\mathrm{P}} / \omega_{\mathrm{T}}=0.75, \gamma / \omega_{\mathrm{T}}\right.$ $=0.01 ; \omega_{\mathrm{T}}^{2} d_{\mathrm{A}}^{2} /\left(3 \pi \hbar \varepsilon_{0} c^{3}\right)=10^{-7} ; z_{\mathrm{A}} / \lambda_{\mathrm{T}}=0.0075$ (solid and dashed lines), $z_{\mathrm{A}} / \lambda_{\mathrm{T}}=0.009$ (dotted and dot-dashed lines)]. For comparison, the approximate results obtained by using the bare frequencies in Eqs. (158) and (159) are also displayed (dashed and dot-dashed lines).

nomial conditional equation for $\delta \omega$, which may be solved numerically. Having calculated $\delta \omega$, we may calculate the (position-dependent) decay rate $\Gamma \equiv \Gamma_{1}$. Neglecting the small free-space decay rate, we replace the Green tensor by its scattering part as given by Eq. (G4), hence from Eqs. (127) and (128) we obtain

$$
\Gamma\left(z_{\mathrm{A}}\right)=\frac{4 C}{\hbar z_{\mathrm{A}}^{3}} \frac{\operatorname{Im} \varepsilon\left[\tilde{\omega}_{10}\left(z_{\mathrm{A}}\right)\right]}{\left|\varepsilon\left[\tilde{\omega}_{10}\left(z_{\mathrm{A}}\right)\right]+1\right|^{2}} .
$$

The resonant part of the $\mathrm{CP}$ force on the excited atom in the short-distance limit can be found by taking the derivative of the scattering part of the Green tensor [Eq. (G4)] with respect to $z_{\mathrm{A}}$ and substituting the re- 
sult into Eq. (149) $(l=1)$. We derive $\left(\mathbf{F}_{11}^{\mathrm{r}}=F_{11}^{\mathrm{r}} \mathbf{e}_{z}\right)$

$$
F_{11}^{\mathrm{r}}\left(z_{\mathrm{A}}\right)=-\frac{3 C}{z_{\mathrm{A}}^{4}} \frac{\left|\varepsilon\left[\Omega_{10}\left(z_{\mathrm{A}}\right)\right]\right|^{2}-1}{\left|\varepsilon\left[\Omega_{10}\left(z_{\mathrm{A}}\right)\right]+1\right|^{2}},
$$

where, according to Eq. (138),

$$
\Omega_{10}\left(z_{\mathrm{A}}\right)=\tilde{\omega}_{10}\left(z_{\mathrm{A}}\right)+i \Gamma\left(z_{\mathrm{A}}\right) / 2 .
$$

Using Eq. (156), we see that $\left(\gamma, \Gamma \ll \omega_{\mathrm{T}}\right)$

$$
\varepsilon\left[\Omega_{10}\left(z_{\mathrm{A}}\right)\right]=1+\frac{\omega_{\mathrm{P}}^{2}}{\omega_{\mathrm{T}}^{2}-\tilde{\omega}_{10}^{2}\left(z_{\mathrm{A}}\right)-i\left[\Gamma\left(z_{\mathrm{A}}\right)+\gamma\right] \tilde{\omega}_{10}\left(z_{\mathrm{A}}\right)} .
$$

Equation (160) differs from the perturbative result in two respects. First, the bare atomic transition frequency $\omega_{10}$ is replaced with the (position-dependent) shifted frequency $\tilde{\omega}_{10}$. Second, the absorption parameter $\gamma$ of the
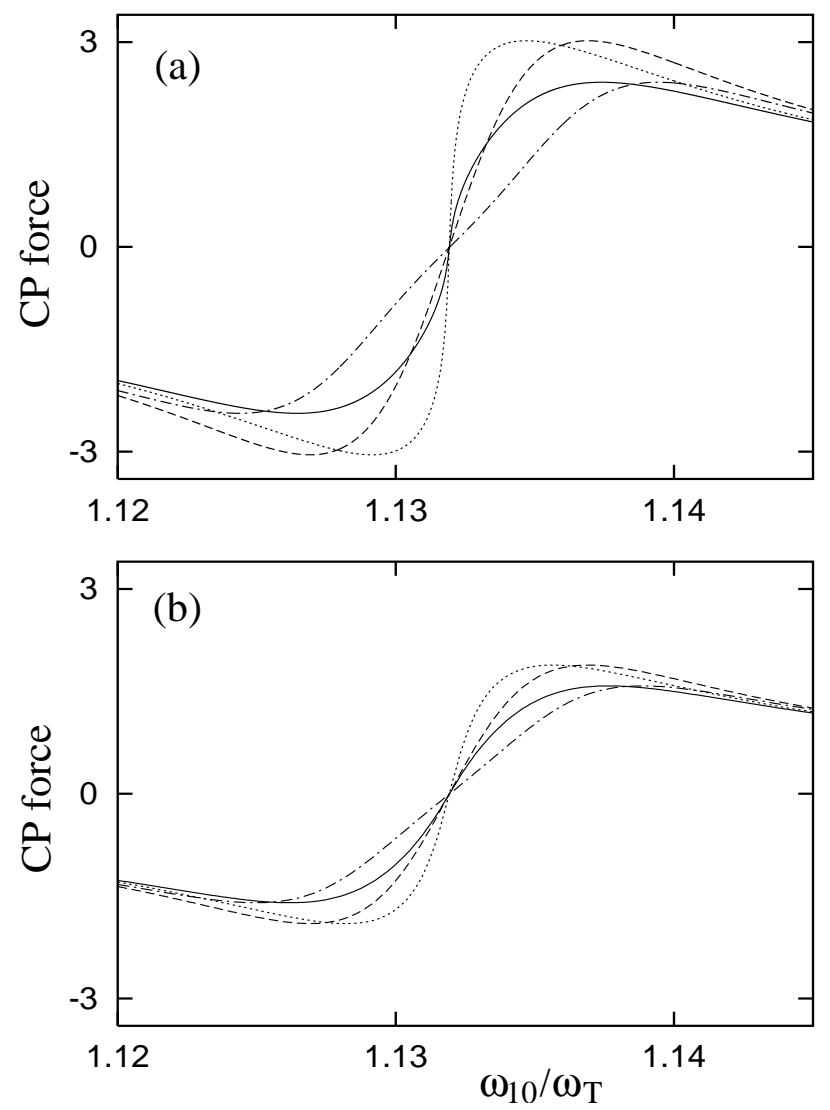

FIG. 2: The resonant part of the CP force $F_{11}^{\mathrm{r}} \lambda_{\mathrm{T}}^{4} \times 10^{-9} /(3 C)$ on a two-level atom that is situated at distance (a) $z_{\mathrm{A}} / \lambda_{\mathrm{T}}=$ 0.0075 and (b) $z_{\mathrm{A}} / \lambda_{\mathrm{T}}=0.009$ of a semi-infinite half space medium of complex permittivity according to Eq. (156) and whose transition dipole moment is perpendicular to the interface (solid lines). The parameters are the same as in Fig. 1 For comparison, both the perturbative result (dashed lines) and the separate effects of level shifting (dotted lines) and level broadening (dash-dotted lines) are shown. medium is replaced with the sum of $\gamma$ and the (positiondependent) atomic decay rate $\Gamma$. The sum $\gamma+\Gamma$ obviously plays the role of the total absorption parameter.

The dependence of $\delta \omega$ and $\Gamma$ on $\omega_{10}$ in the shortdistance limit is shown in Figs. 1(a) and (b), respectively, and Fig. 2 displays the resonant part of the $\mathrm{CP}$ force as a function of $\omega_{10}$. From Fig. [2] it is seen that in the vicinity of the (surface-plasmon induced) frequency $\omega_{\mathrm{S}}=\sqrt{\omega_{\mathrm{T}}^{2}+\omega_{\mathrm{P}}^{2} / 2}$ an enhanced force is observed, which is attractive (repulsive) for red (blue) detuned atomic transition frequencies $\omega_{10}<\omega_{\mathrm{S}}\left(\omega_{10}>\omega_{\mathrm{S}}\right)-$ a result already known from perturbation theory (dashed curves in the figure). However, it is also seen that due to bodyinduced level shifting and broadening the absolute value of the force can be noticeably reduced (solid curves in the figure). Interestingly, the positions of the extrema of the force remain nearly unchanged, because level shifting and broadening give rise to competing effects that almost cancel.

In order to calculate the off-resonant part of the $\mathrm{CP}$ force on the excited atom in the short-distance limit, we first note that, according to Eq. (69),

$$
\begin{gathered}
\boldsymbol{\alpha}_{1}\left(z_{\mathrm{A}}, i u\right)+\boldsymbol{\alpha}_{1}\left(z_{\mathrm{A}},-i u\right)=-\frac{4 \mathbf{d}_{\mathrm{A}} \otimes \mathbf{d}_{\mathrm{A}}}{\hbar} \\
\times \frac{\tilde{\omega}_{10}\left(z_{\mathrm{A}}\right)}{\tilde{\omega}_{10}^{2}\left(z_{\mathrm{A}}\right)+\left[u+\Gamma\left(z_{\mathrm{A}}\right) / 2\right]^{2}} \\
\times \frac{\tilde{\omega}_{10}^{2}\left(z_{\mathrm{A}}\right)+u^{2}+\Gamma^{2}\left(z_{\mathrm{A}}\right) / 4}{\tilde{\omega}_{10}^{2}\left(z_{\mathrm{A}}\right)+\left[u-\Gamma\left(z_{\mathrm{A}}\right) / 2\right]^{2}} .
\end{gathered}
$$

Substituing Eq. (163) into Eq. (148) and making use of Eq. (G7) [where $f(u)$ is given by $u^{2}$ times Eq. (163)], we derive $\left(\mathbf{F}_{11}^{\text {or }}=F_{11}^{\text {or }} \mathbf{e}_{z}\right)$

$$
\begin{aligned}
F_{11}^{\mathrm{or}}\left(z_{\mathrm{A}}\right)= & \frac{3 C}{\pi z_{\mathrm{A}}^{4}} \int_{0}^{\infty} \mathrm{d} u \frac{\varepsilon(i u)-1}{\varepsilon(i u)+1} \\
& \times \frac{\tilde{\omega}_{10}\left(z_{\mathrm{A}}\right)}{\tilde{\omega}_{10}^{2}\left(z_{\mathrm{A}}\right)+\left[u+\Gamma\left(z_{\mathrm{A}}\right) / 2\right]^{2}} \\
& \times \frac{\tilde{\omega}_{10}^{2}\left(z_{\mathrm{A}}\right)+u^{2}+\Gamma^{2}\left(z_{\mathrm{A}}\right) / 4}{\tilde{\omega}_{10}^{2}\left(z_{\mathrm{A}}\right)+\left[u-\Gamma\left(z_{\mathrm{A}}\right) / 2\right]^{2}} .
\end{aligned}
$$

Note that for a two-level atom the relation

$$
F_{00}^{\text {or }}\left(z_{\mathrm{A}}\right)=-F_{11}^{\mathrm{or}}\left(z_{\mathrm{A}}\right)
$$

is valid.

In Fig. 3, the off-resonant part of the CP force is shown as a function of the bare atomic transition frequency. Obviously, the shift of the transition frequency has the effect of raising and lowering the perturbative values of the force (dashed curves) for $\omega_{10}<\omega_{\mathrm{S}}$ and $\omega_{10}>\omega_{\mathrm{S}}$, respectively, which is in full agreement with the frequency response of the frequency shift shown in Fig. 1(a). The influence of the decay rate on the $\mathrm{CP}$ force is extremely weak, as it can be seen from the insets in the figure. This may be understood by the fact that in contrast to the case of the resonant part of the $\mathrm{CP}$ force, where the 

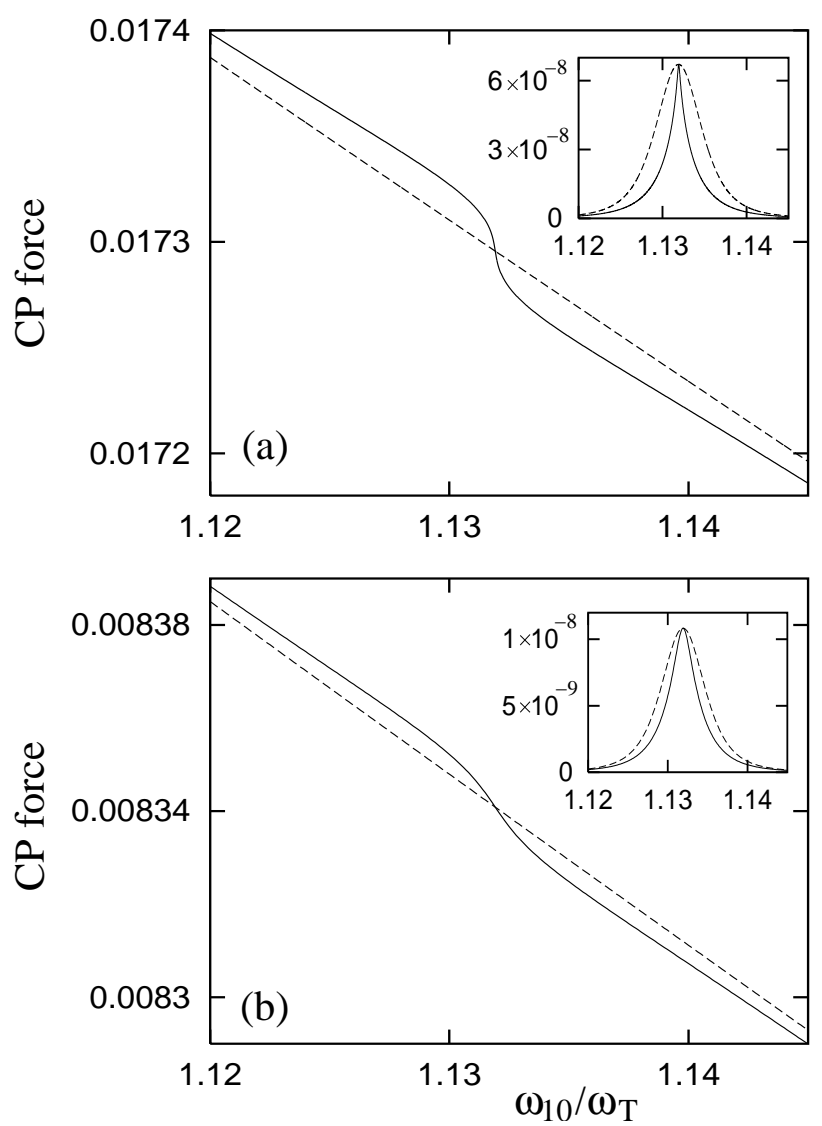

FIG. 3: The off-resonant part of the CP force $F_{11}^{\mathrm{r}} \lambda_{\mathrm{T}}^{4} \times 10^{-9}$ $/(3 C)$ on a two-level atom that is situated at distance (a) $z_{\mathrm{A}} / \lambda_{\mathrm{T}}=0.0075$ and (b) $z_{\mathrm{A}} / \lambda_{\mathrm{T}}=0.009$ of a semi-infinite half space medium of complex permittivity according to Eq. (156) and whose transition dipole moment is perpendicular to the interface (solid lines). The parameters are the same as in Fig. 1. For comparison, the perturbative result (dashed lines) is shown. The insets display the difference between the force with and without consideration of the level broadening (solid lines). For comparison, we show this difference in the case where the level shifts are ignored (dashed lines).

decay rate enters directly via the Green tensor, the influence on the off-resonant part is more indirect via the atomic polarizability. Due to the specific dependence on the atomic polarizability, the leading-order dependence is quadratic in $\Gamma$ and not linear in $\Gamma$ as erroneously predicted from LRT [25]. Physically, the weak influence of the level broadening on the off-resonant part of the $\mathrm{CP}$ force may be regarded as being a consequence of the fact that this part corresponds to energy nonconserving processes (the energy denominators being nonzero), which implies that they happen on (extremely short) time scales where real photon emission does not play a role.

Comparing the magnitudes of the resonant and offresonant components of the $\mathrm{CP}$ force, we see that the off-resonant component is smaller than the resonant one by about two orders of magnitude. However, this observation should be considered with great care. While the two-level atom is a good model for calculating the resonant part of an atom in an excited state, such a simplification is not justified in general when all higher levels can contribute to the off-resonant force component. However, provided that the convergence of the corresponding sum is sufficiently fast, we can still conclude that the resonant part of the $\mathrm{CP}$ force is dominant.

\section{SUMMARY}

Basing on electromagnetic-field quantization that allows for the presence of dispersing and absorbing linear media, and starting with the Lorentz force acting on a neutral atom, we have extended the concept of $\mathrm{CP}$ force beyond the well-known results derived on the basis of normal-mode quantization or LRT in leading order of pertubation theory to allow for (i) magnetodielectric bodies, (ii) an atom that is initially prepared in an arbitrary internal (electronic) quantum state, thereby being subjected to a time-dependent force, (iii) the position dependence of the force via the atomic response, and (iv) arbitrary strength of the atom-field coupling. The basic formulas also apply to the calculation of the radiation forces arising from excited fields such as the force acting on a driven atom.

For a first analysis, we have restricted our attention to a nondriven atom in the weak-coupling regime, so that the internal atomic dynamics can be treated in Markov approximation. It turns out that the force is a superposition of force components weighted by the time-dependent intra-atomic density-matrix elements that solve the intraatomic master equation. Each force component is expressed in terms of the Green tensor of the electromagnetic field and the atomic polarizability, which - through the position-dependent energy level shifts and broadenings - now depends on the position of the atomic system. In consequence even the force components resulting from the electric part of the Lorentz force cannot be derived from potentials in the usual way. Clearly, the position dependence of the atomic polarizability become noticeable only for very small atom-body separations. In order to illustrate the effect, we have considered a two-level atom in the vicinity of a planar semi-infinite medium.

When the atomic system is initially prepared in an eigenstate of its internal Hamiltonian, then only force components associated with diagonal density-matrix elements appear. They solely result from the electric part of the Lorentz force and reduce to the CP forces obtained in lowest-order perturbation theory if the atomic polarizability is replaced with its position-independent perturbative expression. Force components that are associated with excited intra-atomic energy levels are of course transient. As in the course of time an initially excited level is depopulated and lower lying levels are populated, the force that initially acts on the atomic system in the ex- 
cited state changes with time to the force that acts on the atomic system in the ground state.

The results further show that when the atomic system is initially prepared in an intra-atomic quantum state that is a coherent superposition of energy eigenstates, then additional force components associated with the corresponding off-diagonal density-matrix elements are observed. Thus an atomic qubit would typically feel such off-diagonal force components. It should be pointed out that not only the electric but also the magnetic part of the Lorentz force can contribute to the off-diagonal force components, with the magnetic contributions being proportional to the transition frequencies. Clearly, off-diagonal force components are transient.

In contrast to the transient force components that are associated with excited energy levels, off-diagonal force components carry an additional harmonic time dependence. Clearly, if the oscillations are too fast, it can be difficult to detect them experimentally, since they may effectively average to zero. In this case it may be advisable to assign them to the fluctuating part of the force rather than to the average force. The situation may be different in cases where strong atom-field coupling (not considered here) gives rise to Rabi oscillations.

\section{Acknowledgments}

S.Y.B. acknowledges valuable discussions with O. P. Sushkov as well as M.-P. Gorza. This work was supported by the Deutsche Forschungsgemeinschaft. S.Y.B. is grateful for being granted a Thüringer Landesgraduiertenstipendium.

\section{APPENDIX A: DERIVATION OF THE MULTIPOLAR HAMILTONIAN (38)}

To perform transformations of the type

$$
\hat{O}^{\prime}=\hat{U} \hat{O} \hat{U}^{\dagger}
$$

with $\hat{U}$ being given by Eq. (36) together with Eq. (37), we apply the operator identity

$$
e^{\hat{S}} \hat{O} e^{-\hat{S}}=\hat{O}+[\hat{S}, \hat{O}]+\frac{1}{2 !}[\hat{S},[\hat{S}, \hat{O}]]+\ldots
$$

Recalling the commutation relations (4) and (5), it is not difficult to prove that the basic fields $\hat{\mathbf{f}}(\mathbf{r}, \omega)$ are transformed as

$$
\hat{\mathbf{f}}_{\lambda}^{\prime}(\mathbf{r}, \omega)=\hat{\mathbf{f}}_{\lambda}(\mathbf{r}, \omega)+\frac{1}{\hbar \omega} \int \mathrm{d}^{3} r^{\prime} \hat{\mathbf{P}}_{\mathrm{A}}^{\perp}\left(\mathbf{r}^{\prime}\right) \boldsymbol{G}_{\lambda}^{*}\left(\mathbf{r}^{\prime}, \mathbf{r}, \omega\right) .
$$

Using Eq. (A2) together with the commutation relation $\left[\varepsilon_{0} \hat{E}_{k}(\mathbf{r}), \hat{A}_{l}(\mathbf{r})\right]=i \hbar \delta_{k l}^{\perp}\left(\mathbf{r}-\mathbf{r}^{\prime}\right)$, cf. Ref. [37], we find that

$$
\hat{\mathbf{E}}^{\prime}(\mathbf{r})=\hat{\mathbf{E}}(\mathbf{r})+\frac{1}{\varepsilon_{0}} \hat{\mathbf{P}}_{\mathrm{A}}^{\perp}(\mathbf{r}) .
$$

To transform the momenta of the charged particles, the identities

$$
\begin{aligned}
& \nabla_{\alpha} \delta\left(\mathbf{r}-\hat{\mathbf{r}}_{\mathrm{A}}-\lambda \hat{\overline{\mathbf{r}}}_{\beta}\right) \\
& =\left[(\lambda-1) \frac{m_{\alpha}}{m_{\mathrm{A}}}-\lambda \delta_{\alpha \beta}\right] \boldsymbol{\nabla} \delta\left(\mathbf{r}-\hat{\mathbf{r}}_{\mathrm{A}}-\lambda \hat{\overline{\mathbf{r}}}_{\beta}\right), \\
& \int_{0}^{1} \mathrm{~d} \lambda \hat{\mathbf{r}}_{\alpha} \boldsymbol{\nabla} \delta\left(\mathbf{r}-\hat{\mathbf{r}}_{\mathrm{A}}-\lambda \hat{\mathbf{r}}_{\alpha}\right)=\delta\left(\mathbf{r}-\hat{\mathbf{r}}_{\mathrm{A}}\right)-\delta\left(\mathbf{r}-\hat{\mathbf{r}}_{\alpha}\right), \\
& \int_{0}^{1} \mathrm{~d} \lambda \lambda \hat{\mathbf{r}}_{\alpha} \boldsymbol{\nabla} \delta\left(\mathbf{r}-\hat{\mathbf{r}}_{\mathrm{A}}-\lambda \hat{\mathbf{r}}_{\alpha}\right) \\
& =-\delta\left(\mathbf{r}-\hat{\mathbf{r}}_{\alpha}\right)+\int_{0}^{1} \mathrm{~d} \lambda \delta\left(\mathbf{r}-\hat{\mathbf{r}}_{\mathrm{A}}-\lambda \hat{\overline{\mathbf{r}}}_{\alpha}\right)
\end{aligned}
$$

are helpful. They can be proved with the aid of the definitions (28) and (29), and via (partial) integration with respect to $\lambda$. Using Eqs. (A5)-(A7) we derive

$$
\hat{\mathbf{p}}_{\alpha}^{\prime}=\hat{\mathbf{p}}_{\alpha}-q_{\alpha} \hat{\mathbf{A}}\left(\hat{\mathbf{r}}_{\alpha}\right)-\int \mathrm{d}^{3} r \hat{\boldsymbol{\Xi}}_{\alpha}(\mathbf{r}) \times \hat{\mathbf{B}}(\mathbf{r}),
$$

where $\hat{\boldsymbol{\Xi}}_{\alpha}(\mathbf{r})$ is defined as in Eq. (39). Further, the following quantities remain unchanged under the transformation (A1), because they commute with both $\hat{\mathbf{A}}(\mathbf{r})$ (cf. Ref. [37]) and $\hat{\mathbf{r}}_{\alpha}$,

$$
\begin{aligned}
& \hat{\mathbf{A}}^{\prime}(\mathbf{r})=\hat{\mathbf{A}}(\mathbf{r}), \hat{\mathbf{B}}^{\prime}(\mathbf{r})=\hat{\mathbf{B}}(\mathbf{r}), \hat{\varphi}^{\prime}(\mathbf{r})=\hat{\varphi}(\mathbf{r}) \\
& \hat{\mathbf{r}}_{\alpha}^{\prime}=\hat{\mathbf{r}}_{\alpha}, \hat{\mathbf{r}}_{\mathrm{A}}^{\prime}=\hat{\mathbf{r}}_{\mathrm{A}}, \hat{\rho}_{\mathrm{A}}^{\prime}(\mathbf{r})=\hat{\rho}_{\mathrm{A}}(\mathbf{r}), \hat{\varphi}_{\mathrm{A}}^{\prime}(\mathbf{r})=\hat{\varphi}_{\mathrm{A}}(\mathbf{r}), \\
& \hat{\mathbf{P}}_{\mathrm{A}}^{\prime}(\mathbf{r})=\hat{\mathbf{P}}_{\mathrm{A}}(\mathbf{r}), \hat{\mathbf{\Theta}}_{\alpha}^{\prime}(\mathbf{r})=\hat{\boldsymbol{\Theta}}_{\alpha}(\mathbf{r}), \hat{\mathbf{\Xi}}_{\alpha}^{\prime}(\mathbf{r})=\hat{\mathbf{\Xi}}_{\alpha}(\mathbf{r})
\end{aligned}
$$

Applying the transformation rules (A3), (A8), and (A9) - (A11), we may now express the minimal-coupling Hamiltonian (11) in terms of the transformed variables. Recalling Eq. (21) together with Eqs. (8)-(10) and making use of the relations (19) and

$$
\int_{0}^{\infty} \mathrm{d} \omega \frac{\omega}{c^{2}} \operatorname{Im} \boldsymbol{G}\left(\mathbf{r}, \mathbf{r}^{\prime}, \omega\right)=\frac{\pi}{2} \delta\left(\mathbf{r}-\mathbf{r}^{\prime}\right)
$$

(cf. Ref. [36]), we derive

$$
\begin{aligned}
\hat{H}= & \sum_{\lambda=e, m} \int \mathrm{d}^{3} r \int_{0}^{\infty} \mathrm{d} \omega \hbar \omega \hat{\mathbf{f}}_{\lambda}^{\prime \dagger}(\mathbf{r}, \omega) \hat{\mathbf{f}}_{\lambda}^{\prime}(\mathbf{r}, \omega) \\
& +\frac{1}{2 \varepsilon_{0}} \int \mathrm{d}^{3} r \hat{\mathbf{P}}_{\mathrm{A}}^{\perp \perp}(\mathbf{r}) \hat{\mathbf{P}}_{\mathrm{A}}^{\prime \perp}(\mathbf{r})-\int \mathrm{d}^{3} r \hat{\mathbf{P}}_{\mathrm{A}}^{\prime \perp}(\mathbf{r}) \hat{\mathbf{E}}^{\prime \perp}(\mathbf{r}) \\
& +\sum_{\alpha} \frac{1}{2 m_{\alpha}}\left[\hat{\mathbf{p}}_{\alpha}^{\prime}+\int \mathrm{d}^{3} r \hat{\mathbf{\Xi}}_{\alpha}^{\prime}(\mathbf{r}) \times \hat{\mathbf{B}}^{\prime}(\mathbf{r})\right]^{2} \\
& +\frac{1}{2} \int \mathrm{d}^{3} r \hat{\rho}_{\mathrm{A}}^{\prime}(\mathbf{r}) \hat{\varphi}_{\mathrm{A}}^{\prime}(\mathbf{r})+\int \mathrm{d}^{3} r \hat{\rho}_{\mathrm{A}}^{\prime}(\mathbf{r}) \hat{\varphi}^{\prime}(\mathbf{r}) . \quad(\mathrm{A} 13)
\end{aligned}
$$

In order to simplify the last two terms of Eq. (A13), we recall Eq. (86) as well as $\hat{\mathbf{P}}_{\mathrm{A}}^{\prime \|}(\mathbf{r})=\varepsilon_{0} \boldsymbol{\nabla} \hat{\varphi}_{\mathrm{A}}^{\prime}(\mathbf{r})$ and $\hat{\mathbf{E}}^{\prime \|}(\mathbf{r})$ $=-\nabla \hat{\varphi}^{\prime}(\mathbf{r})$, obtaining with the aid of partial integration

$$
\begin{aligned}
& \frac{1}{2} \int \mathrm{d}^{3} r \hat{\rho}_{\mathrm{A}}^{\prime}(\mathbf{r}) \hat{\varphi}_{\mathrm{A}}^{\prime}(\mathbf{r})+\int \mathrm{d}^{3} r \hat{\rho}_{\mathrm{A}}^{\prime}(\mathbf{r}) \hat{\varphi}^{\prime}(\mathbf{r}) \\
& =\frac{1}{2} \int \mathrm{d}^{3} r \hat{\mathbf{P}}_{\mathrm{A}}^{\prime}(\mathbf{r}) \boldsymbol{\nabla} \hat{\varphi}_{\mathrm{A}}^{\prime}(\mathbf{r})+\int \mathrm{d}^{3} r \hat{\mathbf{P}}_{\mathrm{A}}^{\prime}(\mathbf{r}) \boldsymbol{\nabla} \hat{\varphi}^{\prime}(\mathbf{r}) \\
& =\frac{1}{2 \varepsilon_{0}} \int \mathrm{d}^{3} r \hat{\mathbf{P}}_{\mathrm{A}}^{\prime}(\mathbf{r}) \hat{\mathbf{P}}_{\mathrm{A}}^{\prime \|}(\mathbf{r})-\int \mathrm{d}^{3} r \hat{\mathbf{P}}_{\mathrm{A}}^{\prime}(\mathbf{r}) \hat{\mathbf{E}}^{\prime \prime \prime}(\mathbf{r}) .
\end{aligned}
$$


Combining Eqs. A13 and (A14), and noting that integrals containing mixed products of transverse and longitudinal vector fields vanish, we obtain Eq. (38), where we have made use of Eqs. A10 and A11 and hence dropped the primes of all quantities containing the particle coordinates only.

In the simpler case in which the center-of-mass coordinate is treated as a parameter, the transformation law (A8) changes to

$$
\hat{\mathbf{p}}_{\alpha}^{\prime}=\hat{\mathbf{p}}_{\alpha}-q_{\alpha} \hat{\mathbf{A}}\left(\hat{\mathbf{r}}_{\alpha}\right)-\int \mathrm{d}^{3} r \hat{\boldsymbol{\Theta}}_{\alpha}(\mathbf{r}) \times \hat{\mathbf{B}}(\mathbf{r})
$$

Equations (A3), (A4), and A9 - A11 remain formally the same, provided that the replacement $\hat{\mathbf{r}}_{\mathrm{A}} \mapsto \mathbf{r}_{\mathrm{A}}$ is made.

\section{APPENDIX B: ORDERS OF MAGNITUDE OF INTERACTION TERMS}

To estimate the order of magnitude of atom-field interactions, let us introduce the typical atomic length and energy scales

$$
\begin{aligned}
& a_{0}=\frac{a_{\mathrm{B}}}{Z_{\mathrm{eff}}}=\frac{\hbar}{Z_{\mathrm{eff}} \alpha_{0} m_{e} c} \\
& E_{0}=Z_{\mathrm{eff}}^{2} E_{\mathrm{R}}=\frac{Z_{\mathrm{eff}}^{2} \hbar^{2}}{2 m_{e} a_{\mathrm{B}}^{2}} \approx Z_{\mathrm{eff}}^{2} 13.6 \mathrm{eV}
\end{aligned}
$$

( $a_{\mathrm{B}}$, Bohr radius; $E_{\mathrm{R}}$, Rydberg energy), where $m_{e}$ and $-e$ are the electron mass and charge, respectively, $Z_{\text {eff }} e$ is the typical effective nucleus charge felt by the electrons giving the main contributions to the interaction terms to be calculated, and $\alpha_{0}=e^{2} /\left(4 \pi \varepsilon_{0} \hbar c\right)$ is the fine-structure constant. As a rough estimate we can then make the replacements

$$
\begin{aligned}
& q_{\alpha} \rightarrow e, \quad m_{\alpha} \rightarrow m_{e}, \quad \omega_{k l} \rightarrow E_{0} / \hbar \\
& \hat{\overline{\mathbf{r}}}_{\alpha} \rightarrow a_{0}, \quad \dot{\hat{\mathbf{r}}}_{\mathrm{A}} \rightarrow v, \quad \hat{\overline{\mathbf{p}}}_{\alpha}^{(\prime)} \rightarrow p=m_{e} E_{0} a_{0} / \hbar
\end{aligned}
$$

[for the last replacement, see Eq. (C7)]. With regard to the length scale of variation of the medium-assisted electromagnetic field we may make the replacements

$$
\begin{aligned}
& \nabla \rightarrow \lambda^{-1} \sim \omega / c, \quad \nabla \hat{\varphi} \rightarrow \nabla \varphi \sim \omega A, \\
& \hat{\mathbf{E}}^{(\prime)} \rightarrow E \sim \omega A, \hat{\mathbf{B}}^{(\prime)} \rightarrow B \sim(\omega / c) A
\end{aligned}
$$

$\left(\hat{\mathbf{A}}^{(\prime)} \rightarrow A\right)$. Noting that materials typically become transparent for frequencies that are greater than $20 \mathrm{eV}$ (cf. Ref. [48]),

$$
\varepsilon(\mathbf{r}, \omega) \approx 1 \Rightarrow \boldsymbol{G}^{(1)}\left(\mathbf{r}, \mathbf{r}^{\prime}, \omega\right) \approx 0 \text { for } \hbar \omega \gtrsim 20 \mathrm{eV},
$$

we should require that

$$
\hbar \omega \lesssim 20 \mathrm{eV} \quad \Rightarrow \quad \frac{\hbar \omega}{E_{0}} \lesssim 1
$$

With these approximations at hand, the orders of magnitude of $\Delta_{1} E$ defined by Eq. (55) and $\Delta_{2} E$ defined by Eq. (54) in Sec. III A can be estimated to be

$$
\Delta_{1} E \sim \frac{e^{2} A^{2}}{2 m_{e}}=\frac{e^{2} a_{0}^{2} A^{2}}{\hbar^{2}} E_{0}=g^{2} E_{0}=O\left(g^{2}\right)
$$

and

$$
\begin{array}{r}
\Delta_{2} E \sim \frac{1}{E_{0}+\hbar \omega}\left(\frac{e^{2} p^{2} A^{2}}{m_{e}^{2}}+2 \frac{e a_{0} \nabla \varphi e p A}{m_{e}}+e^{2} a_{0}^{2} \nabla \varphi^{2}\right) \\
=g^{2}\left[1+2\left(\frac{\hbar \omega}{E_{0}}\right)+\left(\frac{\hbar \omega}{E_{0}}\right)^{2}\right] \frac{E_{0}}{1+\hbar \omega / E_{0}}=O\left(g^{2}\right),
\end{array}
$$

where the dimensionless coupling constant

$$
g \equiv e a_{0} A / \hbar
$$

has been introduced. Note that in Eq. (B10) we have approximated $\hat{\mathbf{p}}_{\alpha} \rightarrow p$, because in Sec. III we treat an atom at rest, hence relative and absolute momenta are identical.

In order to give a rough idea of the magnitude of the coupling constant $g$, we need to estimate the magnitude of the field strength $A$. In the context of the current work we consider interactions of an atomic system with the vacuum electromagnetic field, so the relevant quantity is the vacuum fluctuation of the field strength. Recalling Eqs. (8) and (21) and making use of the commutation relations (4) and (5) as well as the integral relation (19), we find

$$
\begin{gathered}
\left\langle\left[\Delta \hat{\mathbf{E}}\left(\mathbf{r}_{\mathrm{A}}\right)\right]^{2}\right\rangle=\left\langle\{0\}\left|\hat{\mathbf{E}}^{2}\left(\mathbf{r}_{\mathrm{A}}\right)\right|\{0\}\right\rangle-\left\langle\{0\}\left|\hat{\mathbf{E}}\left(\mathbf{r}_{\mathrm{A}}\right)\right|\{0\}\right\rangle^{2} \\
=\frac{\hbar}{\pi \varepsilon_{0}} \int_{0}^{\infty} \mathrm{d} \omega^{\prime} \frac{\omega^{\prime 2}}{c^{2}} \operatorname{Im} \operatorname{Tr} \boldsymbol{G}\left(\mathbf{r}_{\mathrm{A}}, \mathbf{r}_{\mathrm{A}}, \omega^{\prime}\right) .
\end{gathered}
$$

When the atomic system is placed sufficiently far away from all macroscopic bodies, a good estimate for the integral can be given by using the vacuum Green tensor $\operatorname{Im} \boldsymbol{G}^{(0)}(\mathbf{r}, \mathbf{r}, \omega)=\omega /(6 \pi c) \boldsymbol{I}$, leading to

$$
\left\langle\left[\Delta \hat{\mathbf{E}}\left(\mathbf{r}_{\mathrm{A}}\right)\right]^{2}\right\rangle \sim \frac{\hbar \omega^{4}}{6 \pi^{2} \varepsilon_{0} c^{3}},
$$

where $\omega$ is a characteristic frequency contributing to the interaction, cf. Eq. (B8). Hence making the replacement

$$
A \sim \sqrt{\frac{\hbar \omega^{2}}{6 \pi^{2} \varepsilon_{0} c^{3}}}
$$

[cf. Eq. (B6)], we find

$$
g \sim Z_{\mathrm{eff}} \sqrt{\frac{\alpha_{0}}{6 \pi}}\left(\frac{\hbar \omega}{E_{0}}\right) \alpha_{0} \sim 10^{-2}
$$

depending on the specific atomic system considered and the characteristic frequencies of the medium. When the atom is situated close to some macroscopic body, the 
scattering Green tensor becomes much larger than the vacuum Green tensor, and the approximation leading to Eq. (B13) is not valid anymore. The increased value of the coupling constant $g$ is reflected by the failure of the perturbative result for small atom-surface separations.

The orders of magnitude of the contributions of the three terms in Eq. (52) to the eigenvalue shift in Sec. III B can be estimated according to

$$
\begin{gathered}
\frac{\left|\hat{\mathbf{d}} \hat{\mathbf{E}}^{\prime}\left(\mathbf{r}_{\mathrm{A}}\right)\right|^{2}}{\hbar\left(\omega_{k l}+\omega\right)} \sim\left(e a_{0} E\right)^{2} \frac{E_{0}}{1+\hbar \omega / E_{0}} \\
=g^{2}\left(\frac{\hbar \omega}{E_{0}}\right)^{2} \frac{E_{0}}{1+\hbar \omega / E_{0}}=O\left(g^{2}\right), \\
\frac{\left|\sum_{\alpha} \frac{q_{\alpha}}{2 m_{\alpha}} \hat{\mathbf{p}}_{\alpha}^{\prime} \times \hat{\overline{\mathbf{r}}}_{\alpha} \hat{\mathbf{B}}^{\prime}\left(\mathbf{r}_{\mathrm{A}}\right)\right|^{2}}{\hbar\left(\omega_{k l}+\omega\right)} \\
\sim\left(\frac{e a_{0} p B}{2 m_{e}}\right)^{2} \frac{E_{0}}{1+\hbar \omega / E_{0}}=\left(Z_{\mathrm{eff}} \alpha_{0} g\right)^{2} \frac{1}{4}\left(\frac{\hbar \omega}{E_{0}}\right)^{2} \\
\times \frac{E_{0}}{1+\hbar \omega / E_{0}}=O\left[\left(Z_{\mathrm{eff}} \alpha_{0} g\right)^{2}\right], \\
\sum_{\alpha} \frac{q_{\alpha}^{2}}{8 m_{\alpha}}\left|\hat{\overline{\mathbf{r}}}_{\alpha} \times \hat{\mathbf{B}}^{\prime}\left(\mathbf{r}_{\mathrm{A}}\right)\right|^{2} \sim \frac{\left(e a_{0} B\right)^{2}}{8 m_{e}} \\
=\left(Z_{\mathrm{eff}} \alpha_{0} g\right)^{2} \frac{1}{8}\left(\frac{\hbar \omega}{E_{0}}\right)^{2} E_{0}=O\left[\left(Z_{\mathrm{eff}} \alpha_{0} g\right)^{2}\right] . \quad(\mathrm{B} 18)
\end{gathered}
$$

Next, let us estimate the orders of magnitude of the various contributions to the Lorentz force given in Sec. IVA The magnitudes of the first and third terms in curly brackets in Eq. (97) can be approximated according to

$$
\begin{aligned}
& \left|\hat{\mathbf{d}} \hat{\mathbf{E}}\left(\hat{\mathbf{r}}_{\mathrm{A}}\right)\right| \sim e a_{0} E=g(\hbar \omega)=O(g), \\
& \frac{1}{2}\left|\dot{\mathbf{r}}_{\mathrm{A}} \hat{\mathbf{B}}^{(\prime)}\left(\hat{\mathbf{r}}_{\mathrm{A}}\right) \times \hat{\mathbf{d}}\right| \\
& \quad \sim \frac{1}{2} e a_{0} B v=\frac{1}{2}\left(\frac{v}{c} g\right)(\hbar \omega)=O(g v / c) .
\end{aligned}
$$

In order to estimate the magnitude of the second term, we make use of the relation

$$
m_{\alpha} \dot{\hat{\mathbf{r}}}_{\alpha}=\hat{\mathbf{p}}_{\alpha}-q_{\alpha} \hat{\mathbf{A}}\left(\hat{\mathbf{r}}_{\alpha}\right)
$$

in order to introduce relative momenta [recall Eq. (49)], leading to

$$
\begin{aligned}
& \sum_{\alpha} \frac{q_{\alpha}}{2} \dot{\hat{\mathbf{r}}}_{\alpha} \hat{\mathbf{B}}\left(\mathbf{r}_{\mathrm{A}}\right) \times \hat{\overline{\mathbf{r}}}_{\alpha}=\sum_{\alpha} \frac{q_{\alpha}}{2 m_{\alpha}} \hat{\overline{\mathbf{p}}}_{\alpha} \hat{\mathbf{B}}\left(\hat{\mathbf{r}}_{\mathrm{A}}\right) \times \hat{\overline{\mathbf{r}}}_{\alpha} \\
& +\sum_{\alpha} \frac{q_{\alpha}^{2}}{2 m_{\alpha}} \hat{\mathbf{r}}_{\alpha} \hat{\mathbf{B}}\left(\hat{\mathbf{r}}_{\mathrm{A}}\right) \times \hat{\mathbf{A}}\left(\hat{\mathbf{r}}_{\mathrm{A}}\right)+\frac{1}{2} \dot{\hat{\mathbf{r}}}_{\mathrm{A}} \hat{\mathbf{B}}\left(\hat{\mathbf{r}}_{\mathrm{A}}\right) \times \hat{\mathbf{d}} .
\end{aligned}
$$

Combining this with

$$
\begin{aligned}
& \left|\sum_{\alpha} \frac{q_{\alpha}}{2 m_{\alpha}} \hat{\overline{\mathbf{p}}}_{\alpha} \hat{\mathbf{B}}\left(\hat{\mathbf{r}}_{\mathrm{A}}\right) \times \hat{\mathbf{r}}_{\alpha}\right| \\
& \sim \frac{e B a_{0} p}{2 m_{e}}=\left(Z_{\mathrm{eff}} \alpha_{0} g\right) \frac{1}{4}(\hbar \omega)=O\left(Z_{\mathrm{eff}} \alpha_{0} g\right) \\
& \left|\sum_{\alpha} \frac{q_{\alpha}^{2}}{2 m_{\alpha}} \hat{\mathbf{r}}_{\alpha} \hat{\mathbf{B}}\left(\hat{\mathbf{r}}_{\mathrm{A}}\right) \times \hat{\mathbf{A}}\left(\hat{\mathbf{r}}_{\mathrm{A}}\right)\right| \\
& \sim \frac{e^{2} a_{0} A B}{2 m_{e}}=\left(Z_{\mathrm{eff}} \alpha_{0} g^{2}\right) \frac{1}{2}(\hbar \omega)=O\left(Z_{\mathrm{eff}} \alpha_{0} g^{2}\right),
\end{aligned}
$$

and Eq. (B20), we see that the magnitude of the second term in curly brackets in Eq. (97) is $O\left(Z_{\mathrm{eff}} \alpha_{0} g+\right.$ $\left.Z_{\text {eff }} \alpha_{0} g^{2}+g v / c\right)=O\left[\left(Z_{\text {eff }} \alpha_{0}+v / c\right) g\right]$. The magnitudes of the different contributions to Eq. (98) are

$$
\begin{gathered}
\left|\dot{\hat{\mathbf{d}}} \times \hat{\mathbf{B}}\left(\hat{\mathbf{r}}_{\mathrm{A}}\right)\right|=\left|\sum_{\alpha} \frac{q_{\alpha}}{m_{\alpha}}\left[\hat{\overline{\mathbf{p}}}_{\alpha}-q_{\alpha} \hat{\mathbf{A}}\left(\hat{\mathbf{r}}_{\mathrm{A}}\right)\right] \times \hat{\mathbf{B}}\left(\hat{\mathbf{r}}_{\mathrm{A}}\right)\right| \\
\sim\left(\frac{e p B}{m_{e}}+\frac{e^{2} A B}{m_{e}}\right)=g(1+2 g)\left(\frac{\omega E_{0}}{c}\right)=O(g) \\
\left|\hat{\mathbf{d}} \times \dot{\hat{\mathbf{B}}}\left(\hat{\mathbf{r}}_{\mathrm{A}}\right)\right| \\
\sim e a_{0} \omega B=g\left(\frac{\hbar \omega}{E_{0}}\right)\left(\frac{\omega E_{0}}{c}\right)=O(g), \\
\frac{1}{2}\left|\hat{\mathbf{d}} \times\left[\dot{\hat{\mathbf{r}}}_{\mathrm{A}} \nabla_{\mathrm{A}} \otimes \hat{\mathbf{B}}\left(\hat{\mathbf{r}}_{\mathrm{A}}\right)+\hat{\mathbf{B}}\left(\hat{\mathbf{r}}_{\mathrm{A}}\right) \otimes \overleftarrow{\nabla}_{\mathrm{A}} \dot{\hat{\mathbf{r}}}_{\mathrm{A}}\right]\right| \\
\sim \frac{e a_{0} v \omega B}{c}=\left(\frac{v}{c} g\right)\left(\frac{\hbar \omega}{E_{0}}\right)\left(\frac{\omega E_{0}}{c}\right)=O(g v / c) .
\end{gathered}
$$

Finally, let us compare the contributions of the Röntgen interaction to the temporal evolution $\hat{\mathbf{f}}_{\lambda}(\mathbf{r}, \omega, t)$ with that from the electric dipole interaction,

$$
\begin{gathered}
\frac{\left|\frac{1}{2 \hbar \omega} \dot{\hat{\mathbf{r}}}_{\mathrm{A}}(t) \hat{\mathbf{d}}(t) \times\left(\nabla_{\mathrm{A}} \times \boldsymbol{G}_{\lambda}^{*}\left[\mathbf{r}_{\mathrm{A}}(t), \mathbf{r}, \omega\right]\right)\right|}{\left|\frac{i}{\hbar} \hat{\mathbf{d}}(t) \boldsymbol{G}_{\lambda}^{*}\left[\hat{\mathbf{r}}_{\mathrm{A}}(t), \mathbf{r}, \omega\right]\right|} \\
\sim\left(\frac{v e a_{0}}{\hbar c}\right) /\left(\frac{e a_{0}}{\hbar}\right)=O(v / c),
\end{gathered}
$$

$$
\begin{gathered}
\frac{\left|\frac{1}{\hbar \omega m_{\mathrm{A}}} \hat{\mathbf{d}}(t) \times \hat{\mathbf{B}}\left[\mathbf{r}_{\mathrm{A}}(t), t\right] \hat{\mathbf{d}}(t) \times\left(\nabla_{\mathrm{A}} \times \boldsymbol{G}_{\lambda}^{*}\left[\mathbf{r}_{\mathrm{A}}(t), \mathbf{r}, \omega\right]\right)\right|}{\left|\frac{i}{\hbar} \hat{\mathbf{d}}(t) \boldsymbol{G}_{\lambda}^{*}\left[\hat{\mathbf{r}}_{\mathrm{A}}(t), \mathbf{r}, \omega\right]\right|} \\
\sim\left(\frac{e^{2} a_{0}^{2} B}{\hbar m_{\mathrm{A}} c}\right) /\left(\frac{e a_{0}}{\hbar}\right)=\left(Z_{\mathrm{eff}} \alpha_{0}\right)^{2} g \frac{1}{2}\left(\frac{\hbar \omega}{E_{0}}\right)\left(\frac{m_{e}}{m_{\mathrm{A}}}\right) \\
=O\left[\left(Z_{\mathrm{eff}} \alpha_{0}\right)^{2} g\right] .
\end{gathered}
$$




\section{APPENDIX C: CALCULATION OF THE PERTURBATIVE CORRECTIONS (58) AND (79)}

Recalling Eq. (6) together with Eqs. (8)-(10), making use of the commutation relations (44) and (5), and applying Eq. (19), Eq. (55) leads to

$$
\begin{aligned}
\Delta_{1} E_{l} & =\sum_{\alpha} \frac{q_{\alpha}^{2}}{2 m_{\alpha}} \sum_{\lambda=e, m} \int_{0}^{\infty} \mathrm{d} \omega \\
& \times \int \mathrm{d}^{3} r \frac{1}{\omega^{2}}\left({ }^{\perp} G_{\lambda}\right)_{i j}\left(\mathbf{r}_{\mathrm{A}}, \mathbf{r}, \omega\right)\left({ }^{\perp} G_{\lambda}^{*}\right)_{i j}\left(\mathbf{r}_{\mathrm{A}}, \mathbf{r}, \omega\right) \\
= & \frac{\hbar \mu_{0}}{\pi} \sum_{\alpha} \frac{q_{\alpha}^{2}}{2 m_{\alpha}} \int_{0}^{\infty} \mathrm{d} \omega \operatorname{Im}\left({ }^{\perp} G^{\perp}\right)_{i i}\left(\mathbf{r}_{\mathrm{A}}, \mathbf{r}_{\mathrm{A}}, \omega\right),(\mathrm{C} 1)
\end{aligned}
$$

where we have introduced the notation

$$
\begin{aligned}
& { }^{\perp(\|)} \boldsymbol{G}^{\perp(\|)}\left(\mathbf{r}, \mathbf{r}^{\prime}, \omega\right) \\
& \quad \equiv \int \mathrm{d}^{3} s \int \mathrm{d}^{3} s^{\prime} \boldsymbol{\delta}^{\perp(\|)}(\mathbf{r}-\mathbf{s}) \boldsymbol{G}\left(\mathbf{s}, \mathbf{s}^{\prime}, \omega\right) \boldsymbol{\delta}^{\perp(\|)}\left(\mathbf{s}^{\prime}-\mathbf{r}^{\prime}\right) .
\end{aligned}
$$

Applying the sum rule

$$
\sum_{\alpha} \frac{q_{\alpha}^{2}}{2 m_{\alpha}} \boldsymbol{I}=\frac{1}{2 \hbar} \sum_{k} \omega_{k l}\left(\mathbf{d}_{l k} \otimes \mathbf{d}_{k l}+\mathbf{d}_{k l} \otimes \mathbf{d}_{l k}\right),
$$

we can rewrite Eq. (C1) as

$$
\Delta_{1} E_{l}=\frac{\mu_{0}}{\pi} \sum_{k} \int_{0}^{\infty} \mathrm{d} \omega \omega_{k l} \mathbf{d}_{l k} \operatorname{Im}^{\perp} G^{\perp}\left(\mathbf{r}_{\mathrm{A}}, \mathbf{r}_{\mathrm{A}}, \omega\right) \mathbf{d}_{k l}
$$

To calculate $\Delta_{2} E$, as given by Eq. (54), we first calculate the matrix elements therein. Recalling Eqs. (44)-(10), we obtain

$$
\begin{aligned}
&\left\langle l\left|\left\langle\{0\}\left|\hat{\mathbf{d}} \boldsymbol{\nabla} \hat{\varphi}(\mathbf{r})_{\mathbf{r}=\mathbf{r}_{\mathrm{A}}}\right|\left\{\mathbf{1}_{\lambda}(\mathbf{r}, \omega)\right\}\right\rangle\right| k\right\rangle \\
&=-\mathbf{d}_{l k} \| \boldsymbol{G}_{\lambda}\left(\mathbf{r}_{\mathrm{A}}, \mathbf{r}, \omega\right), \\
&-\left\langle l\left|\left\langle\{0\}\left|\sum_{\alpha} \frac{q_{\alpha}}{m_{\alpha}} \hat{\mathbf{p}}_{\alpha} \hat{\mathbf{A}}\left(\mathbf{r}_{\mathrm{A}}\right)\right|\left\{\mathbf{1}_{\lambda}(\mathbf{r}, \omega)\right\}\right\rangle\right| k\right\rangle \\
&=\frac{\omega_{k l}}{\omega} \mathbf{d}_{l k}{ }^{\perp} \boldsymbol{G}_{\lambda}\left(\mathbf{r}_{\mathrm{A}}, \mathbf{r}, \omega\right)
\end{aligned}
$$

where the second matrix element has been obtained by means of the identity

$$
\sum_{\alpha} \frac{q_{\alpha}}{m_{\alpha}}\left\langle l\left|\hat{\mathbf{p}}_{\alpha}\right| k\right\rangle=-i \omega_{k l} \mathbf{d}_{l k}
$$

Substituting Eqs. (C5) and (C6) into Eq. (54), we then derive

$$
\begin{aligned}
& \Delta_{2} E_{l}=-\frac{1}{\hbar} \sum_{k} \sum_{\lambda=e, m} \mathcal{P} \int_{0}^{\infty} \frac{\mathrm{d} \omega}{\omega_{k l}+\omega} \int \mathrm{d}^{3} r\left(d_{l k}\right)_{i} \\
& \quad \times\left(d_{k l}\right)_{j}\left[\left({ }^{\|} G_{\lambda}\right)_{i n}\left(\mathbf{r}_{\mathrm{A}}, \mathbf{r}, \omega\right)\left({ }^{\|} G_{\lambda}^{*}\right)_{j n}\left(\mathbf{r}_{\mathrm{A}}, \mathbf{r}, \omega\right)\right. \\
& \quad-\frac{\omega_{k l}}{\omega}\left({ }^{\|} G_{\lambda}\right)_{i n}\left(\mathbf{r}_{\mathrm{A}}, \mathbf{r}, \omega\right)\left({ }^{\perp} G_{\lambda}^{*}\right)_{j n}\left(\mathbf{r}_{\mathrm{A}}, \mathbf{r}, \omega\right) \\
& \quad-\frac{\omega_{k l}}{\omega}\left({ }^{\perp} G_{\lambda}\right)_{i n}\left(\mathbf{r}_{\mathrm{A}}, \mathbf{r}, \omega\right)\left({ }^{\|} G_{\lambda}^{*}\right)_{j n}\left(\mathbf{r}_{\mathrm{A}}, \mathbf{r}, \omega\right) \\
& \left.+\frac{\omega_{k l}^{2}}{\omega^{2}}\left({ }^{\perp} G_{\lambda}\right)_{i n}\left(\mathbf{r}_{\mathrm{A}}, \mathbf{r}, \omega\right)\left({ }^{\perp} G_{\lambda}^{*}\right)_{j n}\left(\mathbf{r}_{\mathrm{A}}, \mathbf{r}, \omega\right)\right] \\
& =\frac{\mu_{0}}{\pi} \sum_{k} \mathcal{P} \int_{0}^{\infty} \frac{\mathrm{d} \omega}{\omega_{k l}+\omega} \mathbf{d}_{l k}\left\{-\omega^{2} \operatorname{Im}{ }^{\|} \boldsymbol{G}^{\|}\left(\mathbf{r}_{\mathrm{A}}, \mathbf{r}_{\mathrm{A}}, \omega\right)\right. \\
& \quad+\omega_{k l} \omega\left[\operatorname{Im}^{\|} \boldsymbol{G}^{\perp}\left(\mathbf{r}_{\mathrm{A}}, \mathbf{r}_{\mathrm{A}}, \omega\right)+\operatorname{Im}^{\perp} \boldsymbol{G}^{\|}\left(\mathbf{r}_{\mathrm{A}}, \mathbf{r}_{\mathrm{A}}, \omega\right)\right] \\
& \left.\quad-\omega_{k l}^{2} \operatorname{Im}{ }^{\perp} \boldsymbol{G}^{\perp}\left(\mathbf{r}_{\mathrm{A}}, \mathbf{r}_{\mathrm{A}}, \omega\right)\right\} \mathbf{d}_{k l},
\end{aligned}
$$

where we have again made use of the identity (19). Adding Eqs. (C4) and (C8) according to Eq. (57), on using the identity $\boldsymbol{G}={ }^{\perp} \boldsymbol{G}^{\perp}+{ }^{\perp} \boldsymbol{G}^{\|}+{ }^{\|} \boldsymbol{G}^{\perp}+{ }^{\|} \boldsymbol{G}^{\|}$[which directly follows from the definition (C2) together with $\boldsymbol{\delta}(\mathbf{r})=\boldsymbol{\delta}^{\|}(\mathbf{r})+\boldsymbol{\delta}^{\perp}(\mathbf{r})$ ], we eventually arrive at Eq. (58).

The derivation of Eq. (79) is completely analogous. The relevant matrix elements can be calculated with the aid of Eq. (21) together with Eqs. (8)-(10) and the commutation relations (4) and (5), cf. the remarks below Eq. (40). The result is

$$
-\left\langle l\left|\left\langle\left\{0^{\prime}\right\}\left|\hat{\mathbf{d}} \hat{\mathbf{E}}^{\prime}\left(\mathbf{r}_{\mathrm{A}}\right)\right|\left\{\mathbf{1}_{\lambda}^{\prime}(\mathbf{r}, \omega)\right\}\right\rangle\right| k\right\rangle=-\mathbf{d}_{l k} \boldsymbol{G}_{\lambda}\left(\mathbf{r}_{\mathrm{A}}, \mathbf{r}, \omega\right) .
$$

Substituting Eq. (C9) into Eq. (78) yields

$$
\begin{aligned}
& \Delta_{2} E_{l}=-\frac{1}{\hbar} \sum_{k} \sum_{\lambda=e, m} \mathcal{P} \int_{0}^{\infty} \frac{\mathrm{d} \omega}{\omega_{k l}+\omega} \int \mathrm{d}^{3} r \\
& \quad \times\left(d_{l k}\right)_{i}\left(d_{k l}\right)_{j}\left(G_{\lambda}\right)_{i n}\left(\mathbf{r}_{\mathrm{A}}, \mathbf{r}, \omega\right)\left(G_{\lambda}^{*}\right)_{j n}\left(\mathbf{r}_{\mathrm{A}}, \mathbf{r}, \omega\right),
\end{aligned}
$$

from which Eq. (79) follows by means of Eq. (19).

\section{APPENDIX D: EQUIVALENCE OF LORENTZ FORCES (94) AND (105)}

To transform the first term in Eq. (105), we apply the the rule (A4), recall that integrals over mixed products of transverse and longitudinal vector fields vanish, and use the identity for the first term in Eq. A14 as well as 
Eqs. (82) and (103). We thus derive

$$
\begin{aligned}
& \nabla_{\mathrm{A}} \int \mathrm{d}^{3} r\left[\hat{\mathbf{P}}_{\mathrm{A}}(\mathbf{r}) \hat{\mathbf{E}}^{\prime}(\mathbf{r})\right] \\
& =\nabla_{\mathrm{A}} \int \mathrm{d}^{3} r\left[\hat{\mathbf{P}}_{\mathrm{A}}(\mathbf{r}) \hat{\mathbf{E}}(\mathbf{r})\right]+\frac{1}{\varepsilon_{0}} \nabla_{\mathrm{A}} \int \mathrm{d}^{3} r\left[\hat{\mathbf{P}}_{\mathrm{A}}(\mathbf{r}) \hat{\mathbf{P}}_{\mathrm{A}}^{\perp}(\mathbf{r})\right] \\
& =\nabla_{\mathrm{A}} \int \mathrm{d}^{3} r\left[\hat{\mathbf{P}}_{\mathrm{A}}(\mathbf{r}) \hat{\mathbf{E}}(\mathbf{r})\right]+\frac{1}{\varepsilon_{0}} \nabla_{\mathrm{A}} \int \mathrm{d}^{3} r \hat{\mathbf{P}}_{\mathrm{A}}^{2}(\mathbf{r}) \\
& \quad-\nabla_{\mathrm{A}} \int \mathrm{d}^{3} r \hat{\rho}_{\mathrm{A}}(\mathbf{r}) \hat{\varphi}_{\mathrm{A}}(\mathbf{r}) \\
& =\nabla_{\mathrm{A}} \int \mathrm{d}^{3} r \hat{\mathbf{P}}_{\mathrm{A}}(\mathbf{r}) \hat{\mathbf{E}}(\mathbf{r}) .
\end{aligned}
$$

In order to simplify the second term in Eq. (105), we use the definitions (29), (39), (88), and (89) to calculate

$$
\begin{aligned}
\frac{1}{2} \sum_{\alpha} & {\left[\hat{\boldsymbol{\Xi}}_{\alpha}(\mathbf{r}) \times \dot{\hat{\mathbf{r}}}_{\alpha}-\dot{\hat{\mathbf{r}}}_{\alpha} \times \hat{\boldsymbol{\Xi}}_{\alpha}(\mathbf{r})\right] } \\
= & \frac{1}{2} \sum_{\alpha} q_{\alpha}\left[\hat{\boldsymbol{\Theta}}_{\alpha}(\mathbf{r}) \times \dot{\hat{\mathbf{r}}}_{\alpha}-\dot{\hat{\mathbf{r}}}_{\alpha} \times \hat{\boldsymbol{\Theta}}_{\alpha}(\mathbf{r})\right] \\
& \quad-\frac{1}{2} \sum_{\beta} q_{\alpha}\left[\hat{\boldsymbol{\Theta}}_{\alpha}(\mathbf{r}) \times \dot{\hat{\mathbf{r}}}_{\mathrm{A}}-\dot{\hat{\mathbf{r}}}_{\mathrm{A}} \times \hat{\boldsymbol{\Theta}}_{\alpha}(\mathbf{r})\right] \\
& +\frac{1}{2}\left[\hat{\mathbf{P}}_{\mathrm{A}}(\mathbf{r}) \times \dot{\hat{\mathbf{r}}}_{\mathrm{A}}-\dot{\hat{\mathbf{r}}}_{\mathrm{A}} \times \hat{\mathbf{P}}_{\mathrm{A}}(\mathbf{r})\right] \\
= & \hat{\mathbf{M}}_{\mathrm{A}}(\mathbf{r})+\hat{\mathbf{M}}_{\mathrm{R}}(\mathbf{r}) .
\end{aligned}
$$

Consequently, recalling that $\hat{\mathbf{B}}^{\prime}(\mathbf{r})=\hat{\mathbf{B}}(\mathbf{r})$, we may write

$$
\begin{gathered}
\nabla_{\mathrm{A}} \int \mathrm{d}^{3} r \frac{1}{2} \sum_{\alpha}\left[\hat{\boldsymbol{\Xi}}_{\alpha}(\mathbf{r}) \times \dot{\hat{\mathbf{r}}}_{\alpha}-\dot{\hat{\mathbf{r}}}_{\alpha} \times \hat{\boldsymbol{\Xi}}_{\alpha}(\mathbf{r})\right] \hat{\mathbf{B}}^{\prime}(\mathbf{r}) \\
=\nabla_{\mathrm{A}} \int \mathrm{d}^{3} r\left[\hat{\mathbf{M}}_{\mathrm{A}}(\mathbf{r})+\hat{\mathbf{M}}_{\mathrm{R}}(\mathbf{r})\right] \hat{\mathbf{B}}(\mathbf{r})
\end{gathered}
$$

as well as

$$
\frac{\mathrm{d}}{\mathrm{d} t}\left[\int \mathrm{d}^{3} r \hat{\mathbf{P}}_{\mathrm{A}}(\mathbf{r}) \times \hat{\mathbf{B}}^{\prime}(\mathbf{r})\right]=\frac{\mathrm{d}}{\mathrm{d} t}\left[\int \mathrm{d}^{3} r \hat{\mathbf{P}}_{\mathrm{A}}(\mathbf{r}) \times \hat{\mathbf{B}}(\mathbf{r})\right] .
$$

Substituting Eqs. (D1), (D3), and (D4) into Eq. (105), we see that Eq. (105) is equivalent to Eq. (94).

\section{APPENDIX E: EQUATIONS OF MOTION FOR $\hat{\mathbf{f}}_{\lambda}^{\prime}(\mathbf{r}, \omega, t)$}

In electric dipole approximation, the temporal evolution of the basic fields $\hat{\mathbf{f}}_{\lambda}^{\prime}(\mathbf{r}, \omega, t)$ is governed by the Hamiltonian given in Eq. (44) together with Eqs. (45), (46), and (50). Using Eqs. (8) and (21)-(23) (with the unprimed fields being replaced with the primed ones) and applying the commutation relations (4) and (5), we obtain

$$
\begin{aligned}
& \dot{\hat{\mathbf{f}}}_{\lambda}^{\prime}(\mathbf{r}, \omega, t)=\frac{i}{\hbar}\left[\hat{H}, \hat{\mathbf{f}}_{\lambda}^{\prime}(\mathbf{r}, \omega, t)\right] \\
&=-i \omega \hat{\mathbf{f}}_{\lambda}^{\prime}(\mathbf{r}, \omega, t)+\frac{i}{\hbar} \hat{\mathbf{d}}(t) \boldsymbol{G}_{\lambda}^{*}\left[\hat{\mathbf{r}}_{\mathrm{A}}(t), \mathbf{r}, \omega\right] \\
&-\frac{1}{2 \hbar \omega} \dot{\hat{\mathbf{r}}}_{\mathrm{A}}(t) \hat{\mathbf{d}}(t) \times\left(\nabla_{\mathrm{A}} \times \boldsymbol{G}_{\lambda}^{*}\left[\hat{\mathbf{r}}_{\mathrm{A}}(t), \mathbf{r}, \omega\right]\right) \\
&-\frac{1}{\hbar \omega m_{\mathrm{A}}}\left\{\hat{\mathbf{d}}(t) \times \hat{\mathbf{B}}^{\prime}\left[\hat{\mathbf{r}}_{\mathrm{A}}(t), t\right] \hat{\mathbf{d}}(t)\right. \\
&\left.\quad \times\left(\nabla_{\mathrm{A}} \times \boldsymbol{G}_{\lambda}^{*}\left[\hat{\mathbf{r}}_{\mathrm{A}}(t), \mathbf{r}, \omega\right]\right)\right\} .
\end{aligned}
$$

The third and fourth terms in Eq. (E1), which are due to the Röntgen interaction, are smaller than the second one by factors of $v / c$ and $g\left(Z_{\text {eff }} \alpha_{0}\right)^{2}$, respectively [Eqs. (B28) and (B29) in Appendix [B], so according to the nonrelativistic approximation, Eq. (E1) reduces to

$$
\dot{\hat{\mathbf{f}}}_{\lambda}^{\prime}(\mathbf{r}, \omega, t)=-i \omega \hat{\mathbf{f}}_{\lambda}^{\prime}(\mathbf{r}, \omega, t)+\frac{i}{\hbar} \hat{\mathbf{d}}(t) \boldsymbol{G}_{\lambda}^{*}\left[\hat{\mathbf{r}}_{\mathrm{A}}(t), \mathbf{r}, \omega\right],
$$

which can be integrated to yield $\left[\hat{\mathbf{f}}_{\lambda}^{\prime}(\mathbf{r}, \omega, 0) \equiv \hat{\mathbf{f}}_{\lambda}^{\prime}(\mathbf{r}, \omega)\right]$

$$
\hat{\mathbf{f}}_{\lambda}^{\prime}(\mathbf{r}, \omega, t)=\hat{\mathbf{f}}_{\lambda \text { free }}^{\prime}(\mathbf{r}, \omega, t)+\hat{\mathbf{f}}_{\lambda \text { source }}^{\prime}(\mathbf{r}, \omega, t),
$$

where

$$
\hat{\mathbf{f}}_{\lambda \text { free }}^{\prime}(\mathbf{r}, \omega, t)=e^{-i \omega t} \hat{\mathbf{f}}_{\lambda}^{\prime}(\mathbf{r}, \omega)
$$

and

$\hat{\mathbf{f}}_{\lambda \text { source }}^{\prime}(\mathbf{r}, \omega, t)=\frac{i}{\hbar} \int_{0}^{t} \mathrm{~d} t^{\prime} e^{-i \omega\left(t-t^{\prime}\right)} \hat{\mathbf{d}}\left(t^{\prime}\right) \boldsymbol{G}_{\lambda}^{*}\left[\hat{\mathbf{r}}_{\mathrm{A}}\left(t^{\prime}\right), \mathbf{r}, \omega\right]$.

Substituting Eqs. (E3)-(E5) into Eq. (8) $[\underline{\hat{\mathbf{E}}}(\mathbf{r}, \omega, t) \mapsto$ $\left.\underline{\hat{\mathbf{E}}}^{\prime}(\mathbf{r}, \omega, t)\right]$ and using the identity (19), we arrive at Eqs. (109)-111).

\section{APPENDIX F: INTRA-ATOMIC EQUATIONS OF MOTION}

An estimation similar to that given for the fields $\hat{\mathbf{f}}^{\prime}(\mathbf{r}, \omega, t)$ shows that in the nonrelativistic limit the second term in the interaction Hamiltonian in electric dipole approximation (50) can be disregarded in the calculation of the temporal evolution of the intra-atomic operators $\hat{A}_{m n}(t)$. By representing the (unperturbed) intra-atomic Hamiltonian in the form of Eq. (53), recalling Eqs. (8) and (121), and applying standard commutation relations, it is not difficult to prove that the $\hat{A}_{m n}(t)$ obey the equations of motion

$$
\begin{aligned}
& \dot{\hat{A}}_{m n}=\frac{i}{\hbar}\left[\hat{H}, \hat{A}_{m n}\right]=i \omega_{m n} \hat{A}_{m n} \\
& +\frac{i}{\hbar} \sum_{k}\left[\left(\mathbf{d}_{n k} \hat{A}_{m k}-\mathbf{d}_{k m} \hat{A}_{k n}\right) \int_{0}^{\infty} \mathrm{d} \omega \underline{\hat{\mathbf{E}}}^{\prime}\left(\hat{\mathbf{r}}_{\mathrm{A}}, \omega\right)\right. \\
& \left.\quad+\int_{0}^{\infty} \mathrm{d} \omega \underline{\hat{\mathbf{E}}}^{\dagger}\left(\hat{\mathbf{r}}_{\mathrm{A}}, \omega\right)\left(\mathbf{d}_{n k} \hat{A}_{m k}-\mathbf{d}_{k m} \hat{A}_{k n}\right)\right] .
\end{aligned}
$$


We now substitute the source-quantity representation for $\underline{\hat{\mathbf{E}}}^{\prime}\left(\hat{\mathbf{r}}_{\mathrm{A}}, \omega\right)=\underline{\mathbf{E}}^{\prime}\left[\hat{\mathbf{r}}_{\mathrm{A}}(t), \omega, t\right]$ (and its Hermitian conjugate) according to Eqs. (109)-111) into Eq. (F1). Carrying out the time integral in the source-field part in Eq. (F1) in the Markov approximation, we may set, on regarding $\hat{\mathbf{r}}_{\mathrm{A}}=\hat{\mathbf{r}}_{\mathrm{A}}(t)$ as being slowly varying,

$$
\int_{0}^{\infty} \mathrm{d} \omega \underline{\hat{\mathbf{E}}}_{\text {source }}^{\prime}\left(\hat{\mathbf{r}}_{\mathrm{A}}, \omega\right)=\sum_{m, n} \mathbf{g}_{m n}\left(\hat{\mathbf{r}}_{\mathrm{A}}\right) \hat{A}_{m n}
$$

where

$$
\begin{array}{r}
\mathbf{g}_{m n}\left(\hat{\mathbf{r}}_{\mathrm{A}}\right)=\frac{i \mu_{0}}{\pi} \int_{0}^{\infty} \mathrm{d} \omega \omega^{2} \operatorname{Im} \boldsymbol{G}\left(\hat{\mathbf{r}}_{\mathrm{A}}, \hat{\mathbf{r}}_{\mathrm{A}}, \omega\right) \mathbf{d}_{m n} \\
\times \zeta\left[\tilde{\omega}_{n m}\left(\hat{\mathbf{r}}_{\mathrm{A}}\right)-\omega\right]
\end{array}
$$

$[\zeta(x)=\pi \delta(x)+i \mathcal{P} / x]$, with $\tilde{\omega}_{n m}\left(\hat{\mathbf{r}}_{\mathrm{A}}\right)$ being the shifted transition frequencies. Substituting Eq. (F2) into Eq. (ㅍ1), we obtain

$$
\begin{aligned}
\dot{\hat{A}}_{m n}= & \left\{i \omega_{m n}+\frac{i}{\hbar} \sum_{k}\left[\mathbf{d}_{n k} \mathbf{g}_{k n}-\mathbf{d}_{k m} \mathbf{g}_{k m}^{*}\right]\right\} \hat{A}_{m n} \\
& +\hat{B}_{m n}+\hat{F}_{m n}
\end{aligned}
$$

with

$$
\begin{aligned}
\hat{B}_{m n}= & \frac{i}{\hbar} \sum_{k, l \neq n} \mathbf{d}_{n k} \mathbf{g}_{k l} \hat{A}_{m l}-\frac{i}{\hbar} \sum_{k, l} \mathbf{d}_{k m} \mathbf{g}_{n l} \hat{A}_{k l} \\
& +\frac{i}{\hbar} \sum_{k, l} \mathbf{d}_{n k} \mathbf{g}_{m l}^{*} \hat{A}_{l k}-\frac{i}{\hbar} \sum_{k, l \neq m} \mathbf{d}_{k m} \mathbf{g}_{k l}^{*} \hat{A}_{l n}
\end{aligned}
$$

$(m \neq n)$, and

$$
\begin{aligned}
\dot{\hat{A}}_{m m}= & \frac{i}{\hbar} \sum_{k}\left[\mathbf{d}_{m k} \mathbf{g}_{k m}-\mathbf{d}_{k m} \mathbf{g}_{k m}^{*}\right] \hat{A}_{m m} \\
& -\frac{i}{\hbar} \sum_{k}\left[\mathbf{d}_{k m} \mathbf{g}_{m k}-\mathbf{d}_{m k} \mathbf{g}_{m k}^{*}\right] \hat{A}_{k k} \\
& +\hat{C}_{m m}+\hat{F}_{m m},
\end{aligned}
$$

with

$$
\begin{aligned}
\hat{C}_{m m}= & \frac{i}{\hbar} \sum_{k, l \neq m}\left\{\mathbf{d}_{m k} \mathbf{g}_{k l} \hat{A}_{m l}-\mathbf{d}_{k m} \mathbf{g}_{k l}^{*} \hat{A}_{l m}\right\} \\
& -\frac{i}{\hbar} \sum_{k, l \neq k}\left\{\mathbf{d}_{k m} \mathbf{g}_{m l} \hat{A}_{k l}-\mathbf{d}_{n k} \mathbf{g}_{m l}^{*} \hat{A}_{l k}\right\}
\end{aligned}
$$

where $\hat{F}_{m n}$ denotes contributions from the free-field part in Eq. (109). Taking expectation values with respect to the internal atomic motion and the medium-assisted electromagnetic field, with the density-matrix given by Eq. (119), we can use the property (117), finding that the terms $\hat{F}_{m n}$ do not contribute. In the absence of (quasi)degeneracies such that

$$
\left|\tilde{\omega}_{m n}-\tilde{\omega}_{m^{\prime} n^{\prime}}\right| \gg \frac{1}{2}\left|\Gamma_{m}+\Gamma_{n}-\Gamma_{m^{\prime}}-\Gamma_{n^{\prime}}\right|
$$

we may disregard couplings between different off-diagonal transitions and between off-diagonal and diagonal transitions and thus omit the terms $\hat{B}_{m n}$ and $\hat{C}_{m m}$, hence upon using the decomposition

$$
\frac{i}{\hbar} \mathbf{d}_{n k} \mathbf{g}_{k n}\left(\hat{\mathbf{r}}_{\mathrm{A}}\right)=-i \delta \omega_{n}^{k}\left(\hat{\mathbf{r}}_{\mathrm{A}}\right)-\frac{1}{2} \Gamma_{n}^{k}\left(\hat{\mathbf{r}}_{\mathrm{A}}\right)
$$

where $\delta \omega_{n}^{k}\left(\hat{\mathbf{r}}_{\mathrm{A}}\right)$ and $\Gamma_{n}^{k}\left(\hat{\mathbf{r}}_{\mathrm{A}}\right)$, respectively, are defined according to Eqs. (126) and (128) [with $\boldsymbol{G}^{(1)}\left(\hat{\mathbf{r}}_{\mathrm{A}}, \hat{\mathbf{r}}_{\mathrm{A}}, \omega\right)$ instead of $\boldsymbol{G}\left(\hat{\mathbf{r}}_{\mathrm{A}}, \hat{\mathbf{r}}_{\mathrm{A}}, \omega\right)$ in Eq. (126)], Eqs. (F4) and (F6) lead to Eqs. (123), (131), and (132).

\section{APPENDIX G: HALF SPACE MEDIUM}

The equal-position scattering Green tensor for a semiinfinite half space which contains for $z<0$ a homogeneous, dispersing, and absorbing magnetodielectric medium reads for $z>0$ [49]

$$
\begin{aligned}
& \boldsymbol{G}^{(1)}(\mathbf{r}, \mathbf{r}, \omega)=\frac{i}{8 \pi} \int_{0}^{\infty} \mathrm{d} q \frac{q}{\beta_{0}} e^{2 i \beta_{0} z} \\
& \quad \times\left\{r_{s}\left(\begin{array}{lll}
1 & 0 & 0 \\
0 & 1 & 0 \\
0 & 0 & 0
\end{array}\right)+r_{p} \frac{c^{2}}{\omega^{2}}\left(\begin{array}{rrr}
-\beta_{0}^{2} & 0 & 0 \\
0 & -\beta_{0}^{2} & 0 \\
0 & 0 & 2 q^{2}
\end{array}\right)\right\},
\end{aligned}
$$

where

$$
r_{s}=\frac{\mu \beta_{0}-\beta}{\mu \beta_{0}+\beta}, \quad r_{p}=\frac{\varepsilon \beta_{0}-\beta}{\varepsilon \beta_{0}+\beta}
$$

are the reflection coefficients for $s$ - and $p$-polarized waves, respectively $\left(\beta_{0}^{2}=\omega^{2} / c^{2}-q^{2}\right.$ with $\operatorname{Im} \beta_{0}>0, \beta^{2}=\varepsilon \mu \omega^{2} / c^{2}$ $-q^{2}$ with $\left.\operatorname{Im} \beta>0\right)$. For $q \gg|\omega| / c$ and $q \gg \sqrt{|\varepsilon \mu|}|\omega| / c$, respectively, the approximations

$$
\beta_{0} \simeq i q, \quad \beta \simeq i q
$$

can be made. Due to the exponential factor the integration interval is effectively limited to values $q \lesssim 1 / z$. In the short-distance limit $z \sqrt{|\varepsilon \mu|}|\omega| / c \ll 1$, we therefore introduce a small error, if we extrapolate the approximations (G3) to the whole integral, resulting in

$$
\boldsymbol{G}^{(1)}(\mathbf{r}, \mathbf{r}, \omega)=\frac{c^{2}}{32 \pi \omega^{2} z^{3}} \frac{\varepsilon(\omega)-1}{\varepsilon(\omega)+1}\left(\begin{array}{lll}
1 & 0 & 0 \\
0 & 1 & 0 \\
0 & 0 & 2
\end{array}\right)
$$

Note that the magnetic properties of the medium represented by the permeability $\mu$ begin to contribute via terms proportional to $1 / z$. Substitution of Eq. (G4) into the first term of Eq. (129) for $\delta \omega_{n k}=\delta \omega_{10}$ yields Eq. (153).

In order to obtain Eq. (154), we recall Eq. (G1) to 
write

$$
\begin{aligned}
& \int_{0}^{\infty} \mathrm{d} u f(u) \boldsymbol{G}^{(1)}(\mathbf{r}, \mathbf{r}, i u) \\
& \quad=\frac{1}{8 \pi} \int_{0}^{\infty} \mathrm{d} u f(u) \int_{u / c}^{\infty} \mathrm{d} b_{0} e^{-2 b_{0} z} \\
& \quad \times\left\{r_{s}\left(\begin{array}{lll}
1 & 0 & 0 \\
0 & 1 & 0 \\
0 & 0 & 0
\end{array}\right)-r_{p} \frac{c^{2}}{u^{2}}\left(\begin{array}{ccc}
b_{0}^{2} & 0 & 0 \\
0 & b_{0}^{2} & 0 \\
0 & 0 & 2 b_{0}^{2}-\left(\frac{u}{c}\right)^{2}
\end{array}\right)\right\},
\end{aligned}
$$

having changed the integration variable to the imaginary part of $\beta_{0}\left(\beta_{0}=i b_{0}\right)$. Let $\omega_{\mathrm{M}}$ be a characteristic frequency of the medium such that

$$
\varepsilon(i u)-1 \ll 1 \quad \text { for } u>\omega_{\mathrm{M}}
$$

For $u>\omega_{\mathrm{M}}$, the approximation $\beta \sim \beta_{0}$ holds, and consequently the reflection coefficients $r_{s}, r_{p}$ are independent of $b_{0}$. The frequency integral effectively extends up to frequencies of the order $c / z$, hence in the short-range limit $z \omega_{\mathrm{M}} / c \ll 1\left(\Rightarrow c / z \gg \omega_{\mathrm{M}}\right)$ we introduce only a small error by extrapolating this approximation to the whole frequency integral. Performing the $b_{0}$ integral, retaining only leading-order terms in $u z / c$ (in consistency with $z \omega_{\mathrm{M}} / c \ll 1$ ) we derive

$$
\begin{aligned}
& \int_{0}^{\infty} \mathrm{d} u f(u) \boldsymbol{G}^{(1)}(\mathbf{r}, \mathbf{r}, i u) \\
& \quad=-\frac{c^{2}}{32 \pi z^{3}} \int_{0}^{\infty} \mathrm{d} u \frac{f(u)}{u^{2}} \frac{\varepsilon(i u)-1}{\varepsilon(i u)+1}\left(\begin{array}{ccc}
1 & 0 & 0 \\
0 & 1 & 0 \\
0 & 0 & 2
\end{array}\right)
\end{aligned}
$$

Using Eq. (G7) [with $\left.f(u)=u^{2} /\left(\tilde{\omega}_{\mathrm{A}}^{2}+u^{2}\right)\right]$ together with Eq. (129), we obtain Eq. (154).
[1] G. Binnig, C. F. Quate, and Ch. Gerber, Phys. Rev. Lett. 56, 930 (1986).

[2] F. Shimizu and J. Fujita, Phys. Rev. Lett. 88, 123201 (2002).

[3] I. E. Dzyaloshinskii, E. M. Lifshitz, and L. P. Pitaevskii, Adv. Phys. 10, 165 (1961); D. Langbein, Springer Tracts Mod. Phys. 72, 1 (1974); J. Mahanty and B. W. Ninham, Dispersion Forces (Academic, London, 1976); E. A. Hinds, in Advances in Atomic, Molecular, and Optical Physics, edited by D. Bates and B. Bederson (Academic, New York, 1991), Vol. 28, p. 237; P. W. Milonni, The Quantum Vacuum: An Introduction to Quantum Electrodynamics (Academic, San Diego, 1994).

[4] D. Raskin and P. Kusch, Phys. Rev. 179, 712 (1969); A. Shih, D. Raskin, and P. Kusch, Phys. Rev. A 9, 652 (1974); A. Shih and V. A. Parsegian, ibid. 12, 835 (1975).

[5] A. Anderson, S. Haroche, E. A. Hinds, W. Jhe, and D. Meschede, Phys. Rev. A 37, 3594 (1988); C. I. Sukenik, M. G. Boshier, D. Cho, V. Sandoghdar, and E. A. Hinds, Phys. Rev. Lett. 70, 560 (1993).

[6] R. E. Grisenti, W. Schöllkopf, J. P. Toennies, G. C. Hegerfeldt, and T. Köhler, Phys. Rev. Lett. 83, 1755 (1999).

[7] F. Shimizu, Phys. Rev. Lett. 86, 987 (2001); V. Druzhinina and M. DeKieviet, ibid. 91, 193202 (2003).

[8] H. Friedrich, G. Jacoby, and C. G. Meister, Phys. Rev. A 65, 032902 (2002).

[9] A. Landragin, J.-Y. Courtois, G. Labeyrie, N. Vansteenkiste, C. I. Westbrook, and A. Aspect, Phys. Rev. Lett. 77, 1464 (1996).

[10] M. Oria, M. Chevrollier, D. Bloch, M. Fichet, and M. Ducloy, Europhys. Lett. 14, 527 (1991); V. Sandoghdar, C. I. Sukenik, E. A. Hinds, and S. Haroche, Phys. Rev. Lett. 68, 3432 (1992); M. Marrocco, M. Weidinger, R. T. Sang, and H. Walther, ibid. 81, 5784 (1998); H. Failache, S. Saltiel, M. Fichet, D. Bloch, and M. Ducloy, ibid. 83, 5467 (1999); M. A. Wilson, P. Bushev, J. Eschner, F. Schmidt-Kaler, C. Becher, R. Blatt, and U. Dorner, ibid. 91, 213602 (2003).

[11] M. Gorlicki, S. Feron, V. Lorent, and M. Ducloy, Phys.
Rev. A 61, 013603 (1999); R. Marani, L. Cognet, V. Savalli, N. Westbrook, C. I. Westbrook, and A. Aspect, ibid. 61, 053402 (2000).

[12] H. B. G. Casimir and D. Polder, Phys. Rev. 73, 360 (1948).

[13] R. K. Bullough and B. V. Thompson, J. Phys. C 3, 1780 (1970).

[14] M. J. Renne, Physica (Amsterdam) 53, 193 (1971); 56, 124 (1971).

[15] P. W. Milonni and M.-L. Shih, Phys. Rev. A 45, 4241 (1992); see also J. Schwinger, L. L. DeRaad, and K. A. Milton, Ann. Phys. (N. Y.) 115, 1 (1978) for a related treatment.

[16] Y. Tikochinsky and L. Spruch, Phys. Rev. A 48, 4223 (1993); F. Zhou and L. Spruch, ibid. 52, 297 (1995).

[17] M. Boström and B. E. Sernelius, Phys. Rev. A 61, 052703 (2000). Note that the formulas derived in Ref. [16] for frequency-independent, real permittivities are used for studying metals, by allowing for complex permittivities, without any proof.

[18] A. M. Marvin and F. Toigo, Phys. Rev. A 25, 782 (1982). In fact an energy formula based on a normal-mode expansion is combined with elements of LRT.

[19] C.-H. Wu, C.-I. Kuo, and L. H. Ford, Phys. Rev. A 65, 062102 (2002).

[20] S. Y. Buhmann, Ho Trung Dung, and D.-G. Welsch, J. Opt. B: Quantum Semiclassical Opt. 6, 127 (2004).

[21] A. D. McLachlan, Proc. R. Soc. London Ser. A 271, 387 (1963); Mol. Phys. 7, 381 (1963).

[22] G. S. Agarwal, Phys. Rev. A 11, 243 (1975).

[23] J. M. Wylie and J. E. Sipe, Phys. Rev. A 30, 1185 (1984); 32, 2030 (1985).

[24] C. Girard, J. Chem. Phys. 85, 6750 (1986); C. Girard and C. Girardet, ibid. 86, 6531 (1987); C. Girard, S. Maghezzi, and F. Hache, ibid. 91, 5509 (1989).

[25] S. Kryszewski, Mol. Phys. 78, 5, 1225 (1993).

[26] M. Fichet, F. Schuller, D. Bloch, and M. Ducloy, Phys. Rev. A 51, 1553 (1995); M.-P. Gorza, S. Saltiel. H. Failache, and M. Ducloy, Eur. Phys. J. D 15, 113 (2001).

[27] M. Boustimi, J. Baudon, P. Candori, and J. Robert, 
Phys. Rev. B 65, 155402 (2002).

[28] C. Henkel, K. Joulain, J.-P. Mulet, and J.-J. Greffet, J. Opt. A, Pure Appl. Opt. 4, 109 (2002).

[29] Ho Trung Dung, L. Knöll, and D.-G. Welsch, Phys. Rev. A 64, 013804 (2001).

[30] I. V. Bondarev, G. Ya. Slepyan, and S. A. Maksimenko Phys. Rev. Lett. 89, 115504 (2002).

[31] W. P. Healy, J. Phys. A 10, 279 (1977).

[32] C. Baxter, M. Babiker, and R. Loudon, Phys. Rev. A 47, 1278 (1993).

[33] V. E. Lembessis, M. Babiker, C. Baxter, and R. Loudon, Phys. Rev. A 48, 1594 (1993).

[34] M. Wilkens, Phys. Rev. A 47, 671 (1993); 49, 570 (1994).

[35] J.-C. Guillot and J. Robert, J. Phys. A 35, 5023 (2002).

[36] L. Knöll, S. Scheel, and D.-G. Welsch, in Coherence and Statistics of Photons and Atoms, edited by J. Peřina (Wiley, New York, 2001), p. 1; for an update, see arXiv:quant-ph/0006121.

[37] Ho Trung Dung, S. Y. Buhmann, L. Knöll, D.-G. Welsch, S. Scheel, and J. Kästel, Phys. Rev. A 68, 043816 (2003).

[38] V. G. Veselago, Sov. Phys. Usp. 10, 509 (1968).

[39] V. M. Fain and Ya. I. Khanin, Quantum Electronics
(MIT Press, Cambridge MA, 1969). Note that here no distinction is made between bare and shifted transisition frequencies; see also P. W. Milonni and R. W. Boyd, Phys. Rev. A 69, 023814 (2004).

[40] I. B. Khriplovich and S. K. Lamoureaux, CP Violation without Strangeness (Springer, Berlin, 1997).

[41] D. P. Craig and T. Thirunamachandran Molecular Quantum Electrodynamics (Academic Press, New York, 1984).

[42] W. Röntgen, Annu. Rev. Phys. Chem. 35, 264 (1888).

[43] J. P. Gordon, Phys. Rev. A 8, 14 (1973).

[44] R. Loudon, Phys. Rev. A 68, 013806 (2003).

[45] S. Stenholm, Rev. Mod. Phys. 58, 699 (1986).

[46] W. Vogel, D.-G. Welsch, and S. Wallentowitz, Quantum Optics, An Introduction (Wiley-VCH, Berlin, 2001).

[47] L.-M. Duan, J. I. Cirac, and P. Zoller, Science 292, 1695 (2001).

[48] S. Adachi, Optical Constants of Crystalline and Amorphous Semiconductors (Kluwer, Boston, 1999).

[49] W. C. Chew, Waves and Fields in Inhomogeneous Media (IEEE Press, New York, 1995) Secs. 2.1.3, 2.1.4, and 7.4.2. 